\title{
Scalable Process Design for a PDE10A Inhibitor Consisting of Pyrazolopyrimidine and Quinoxaline as Key Units
}

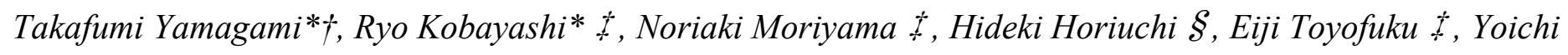

Kadoh II, Eiji Kawanishi 」, Shinichi Izumoto\#, Hajime Hiramatsu\#, Takehiro Nanjo\#, Masuhiro Sugino†,

$$
\text { Masayuki Utsugi†, and Yasunori Moritani† }
$$

$\dagger$ Process Development Department, Production Technology \& Supply Chain Management Division, Mitsubishi Tanabe Pharma Corporation 3-16-89, Kashima, Yodogawa-ku, Osaka 532-8505, Japan,

$$
\text { yamagami.takafumi@mk.mt-pharma.co.jp }
$$

$\$$ Technology Management Department, Production Technology \& Supply Chain Management Division, Mitsubishi Tanabe Pharma Corporation 3-16-89, Kashima, Yodogawa-ku, Osaka 532-8505, Japan,

$$
\text { kobayashi.ryo@mc.mt-pharma.co.jp }
$$

This include spectra of compounds, such as $1,7, \mathbf{8}, 11,12,13,19,20$, and 22, and X-ray structure report for 1. 
Table of contents

1. Spectra of Compound 1 [ ${ }^{1} \mathrm{H}$ NMR, ${ }^{13} \mathrm{C}$ NMR, IR, XRPD] S-3

2. Spectra of Compound $7\left[{ }^{1} \mathrm{H}\right.$ NMR, ${ }^{13} \mathrm{C}$ NMR]

3. Spectra of Compound 8 [ ${ }^{1} \mathrm{H}$ NMR, ${ }^{13} \mathrm{C}$ NMR] S-6

4. Spectra of Compound 11 [ ${ }^{1} \mathrm{H}$ NMR, ${ }^{13} \mathrm{C}$ NMR] S-7

5. Spectra of Compound 12 [ ${ }^{1} \mathrm{H}$ NMR, ${ }^{13} \mathrm{C}$ NMR] S-8

6. Spectra of Compound 13 [ ${ }^{1} \mathrm{H}$ NMR, ${ }^{13} \mathrm{C}$ NMR] S-9

7. Spectra of Compound $19\left[{ }^{1} \mathrm{H}\right.$ NMR, ${ }^{13} \mathrm{C}$ NMR $] \quad$ S-10

8. Spectra of Compound $20\left[{ }^{1} \mathrm{H}\right.$ NMR, ${ }^{13} \mathrm{C}$ NMR] $\quad$ S-11

9. Spectra of Compound 22 [ ${ }^{1} \mathrm{H}$ NMR, ${ }^{13} \mathrm{C}$ NMR] $\quad$ S-12

10. X-ray structure report for Compound $1 \quad S-13$

11. EXPERIMENTAL DETAILS for X-ray structure S-16

Table S1. Atomic coordinates and $\mathrm{B}_{\mathbf{i s o}} / \mathrm{B}_{\mathbf{e q}} \quad \mathrm{S}-20$

Table S2. Atomic coordinates and Biso involving hydrogen atoms S-22

Table S3. Anisotropic displacement parameters $\quad$ S-24

Table S4. Fragment Analysis $\quad$ S-26

$\begin{array}{lr}\text { Table S5. Bond lengths }(\AA) & \text { S-27 }\end{array}$

$\begin{array}{lr}\text { Table S6. Bond lengths involving hydrogens }(\AA) & \text { S-29 }\end{array}$

$\begin{array}{lr}\text { Table S7. Bond angles }\left({ }^{(}\right) & \text {S-30 }\end{array}$

$\begin{array}{lr}\text { Table S8. Bond angles involving hydrogens }\left({ }^{\circ}\right) & \text { S-32 }\end{array}$

Table S9. Torsion Angles( $\left(^{\circ}\right) \quad$ S-34

Table S10. Possible hydrogen bonds $\quad$ S-36

Table S11. Intramolecular contacts less than $3.60 \AA \quad S-37$

Table S12. Intramolecular contacts less than $3.60 \AA$ involving hydrogens $\quad$ S-38

Table S13. Intermolecular contacts less than $3.60 \AA \quad S-43$

Table S14. Intermolecular contacts less than $3.60 \AA \AA$ involving hydrogens $\quad$ S-45 
Compound 1

${ }^{1} \mathrm{H}$ NMR $\left(\mathrm{CD}_{3} \mathrm{OD}\right)$

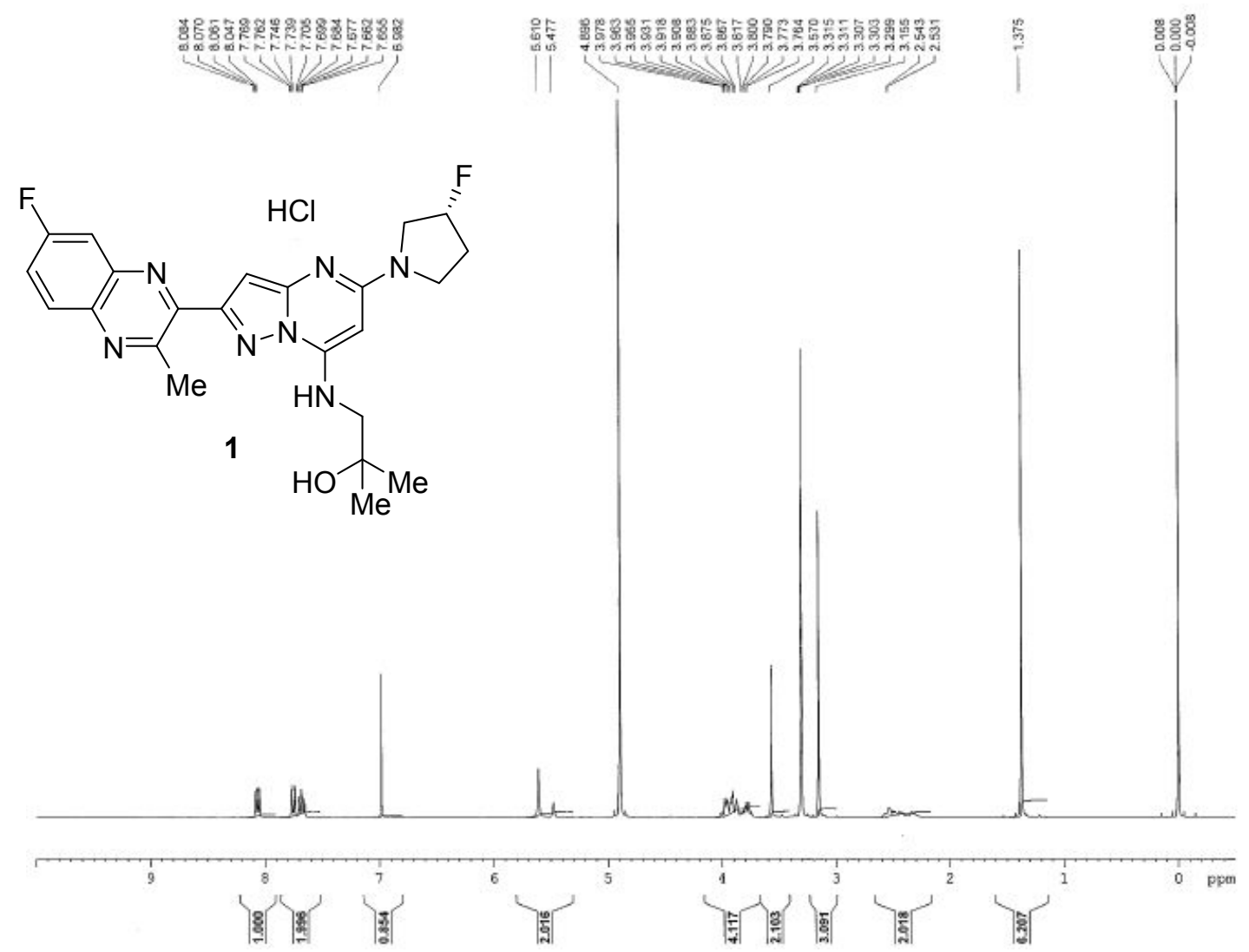

${ }^{13} \mathrm{C}$ NMR $\left(\mathrm{CD}_{3} \mathrm{OD}\right)$

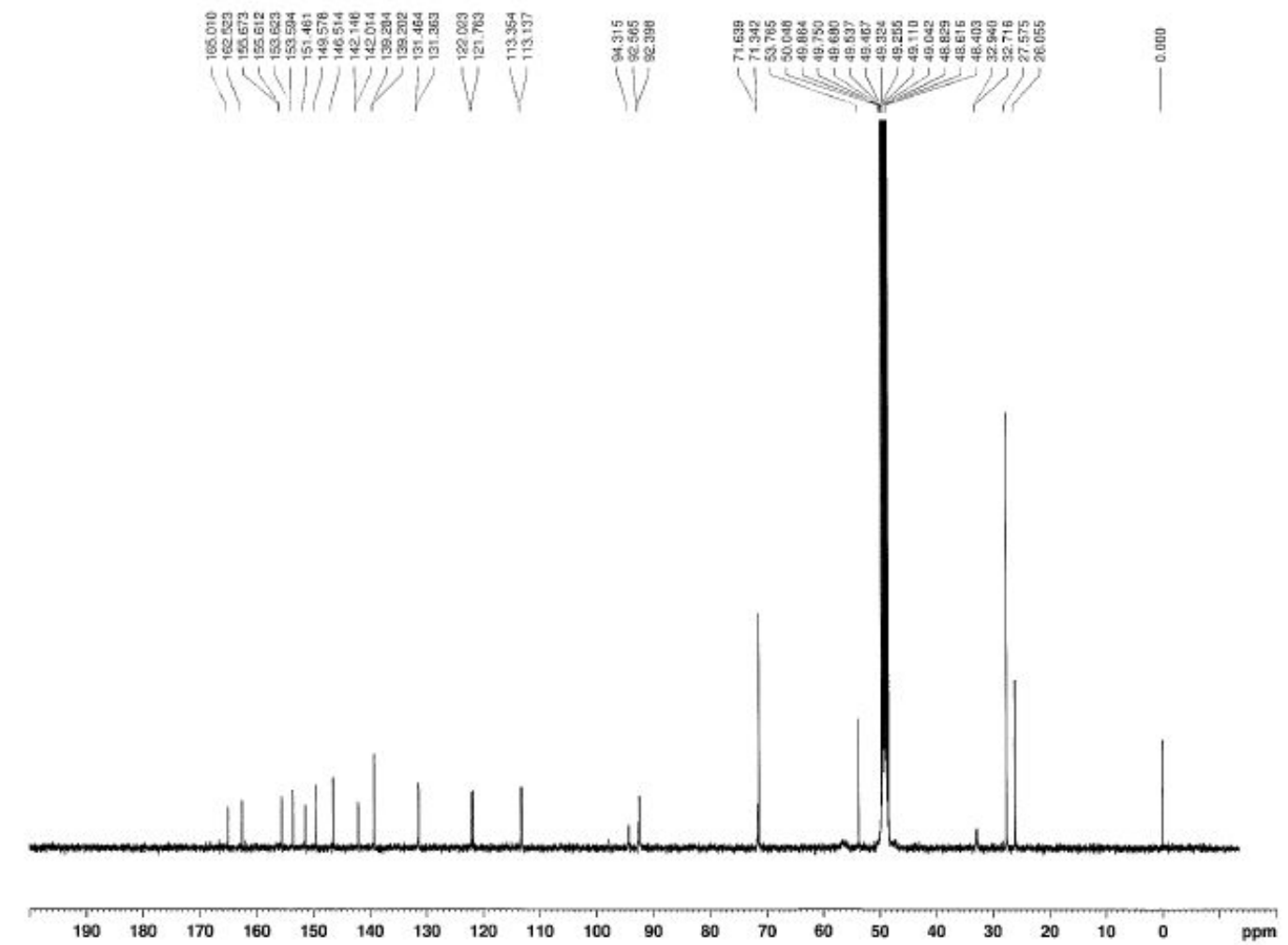


$\mathrm{IR}(\mathrm{KCl})$

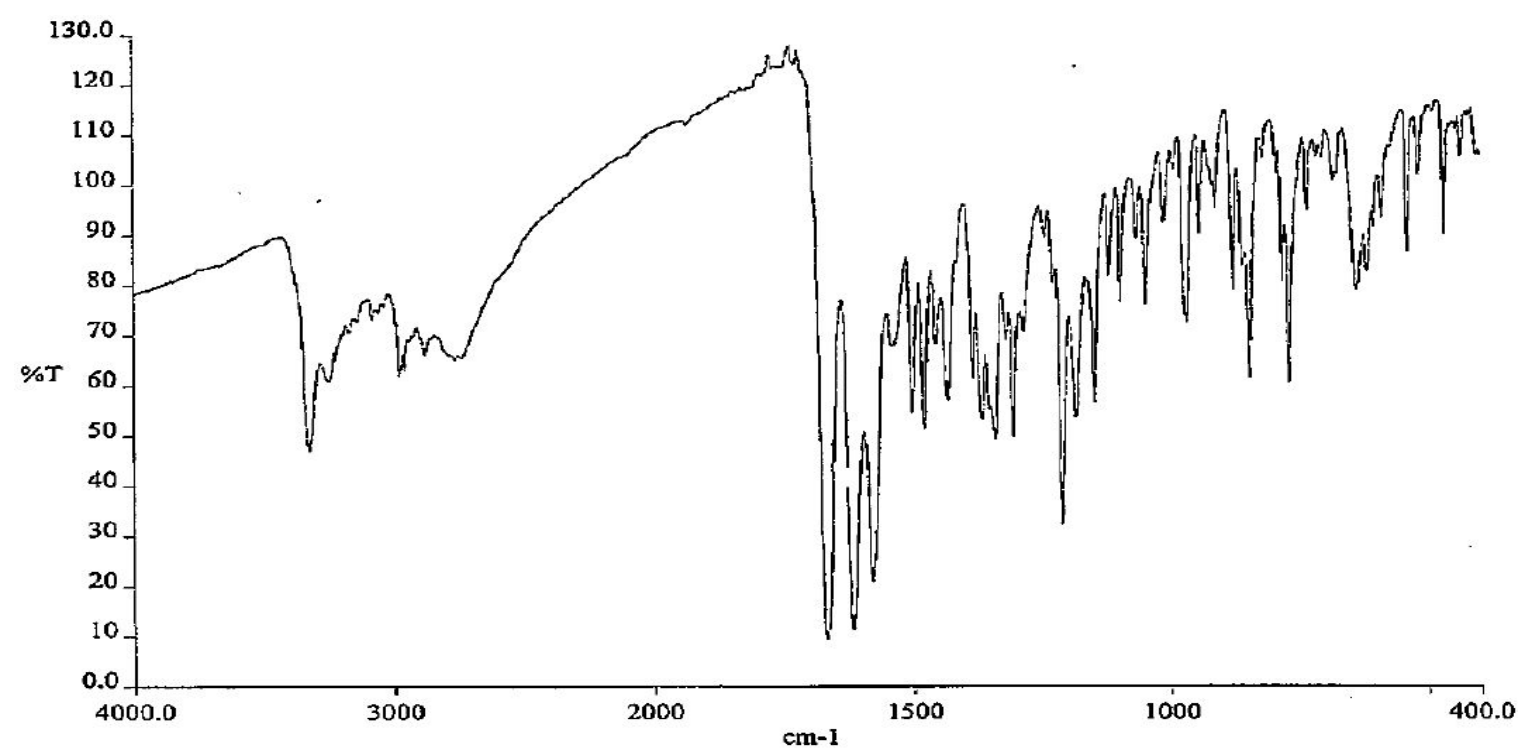

XRPD

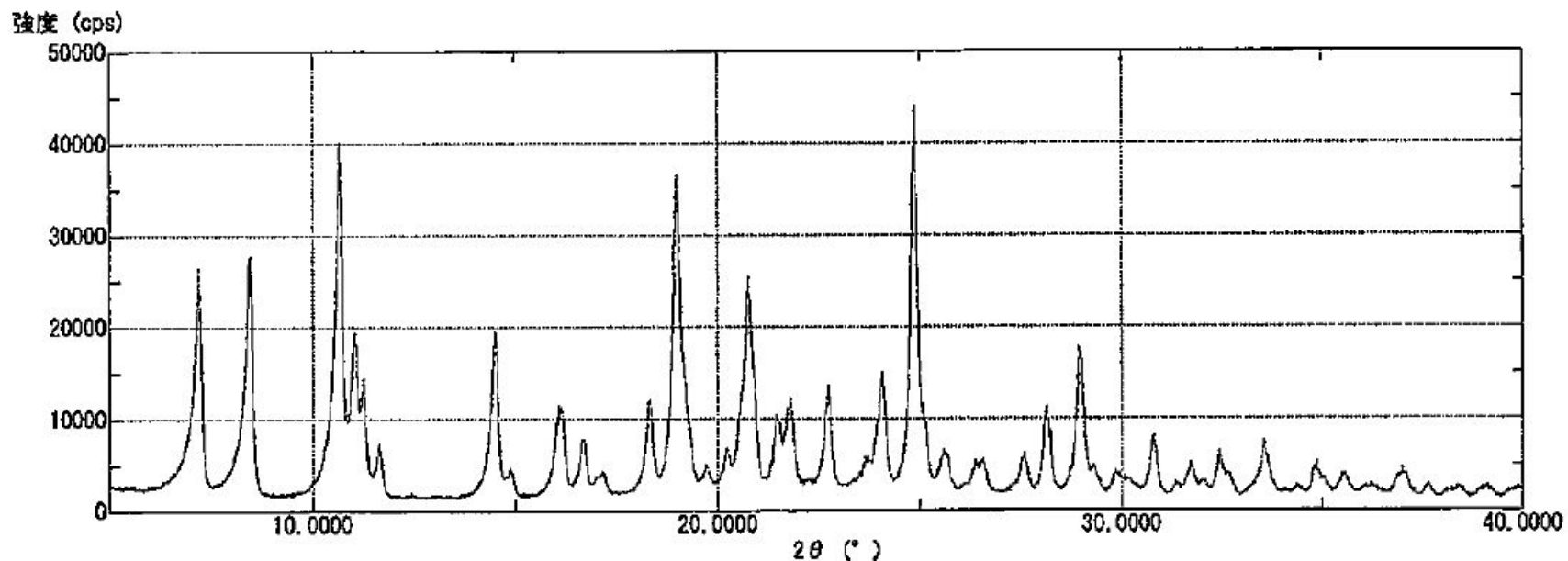

X-ray powder diffraction pattern calculated from the crystal structure of Compound 1

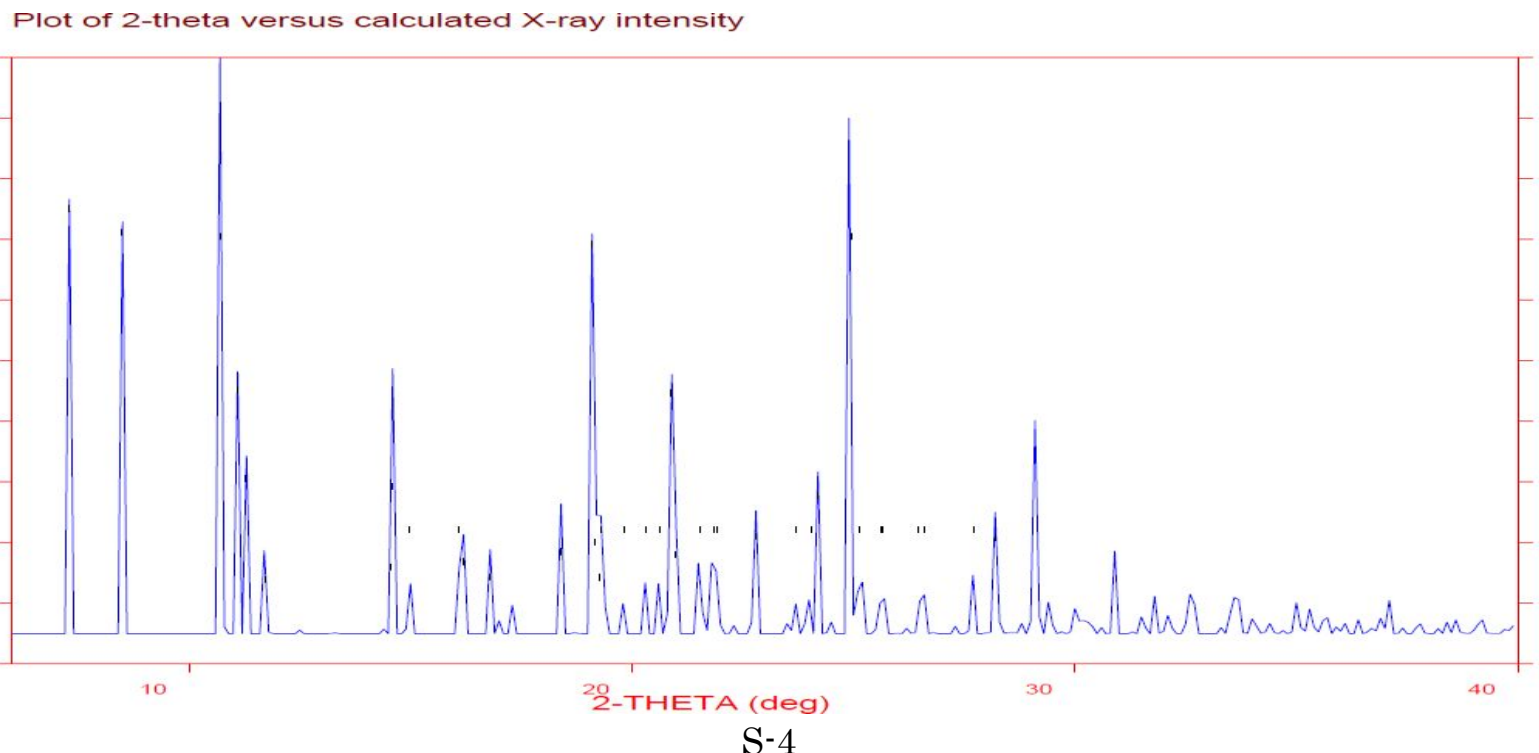


Compound 7
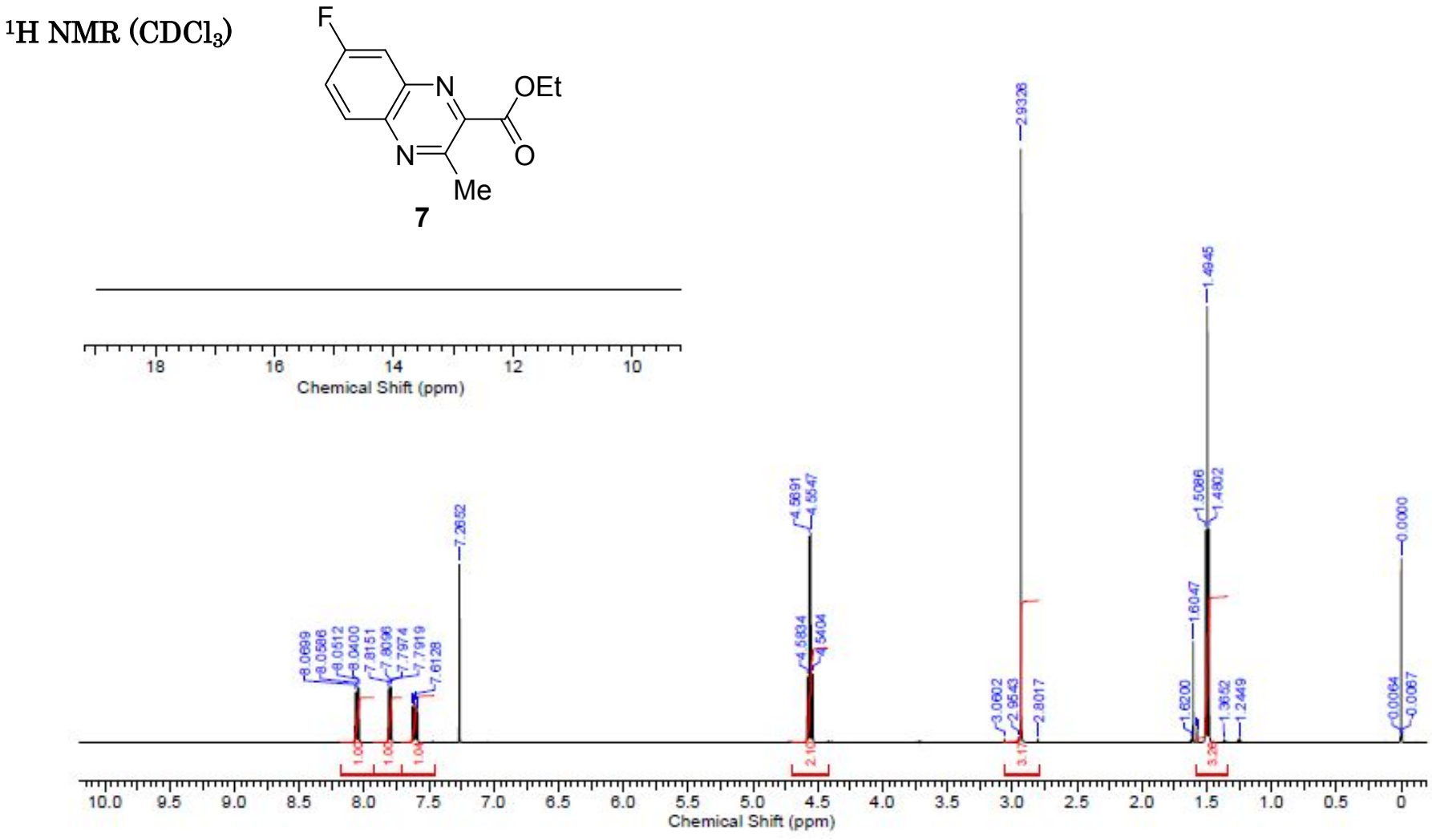

\section{${ }^{13} \mathrm{C} \mathrm{NMR}\left(\mathrm{CDCl}_{3}\right)$}

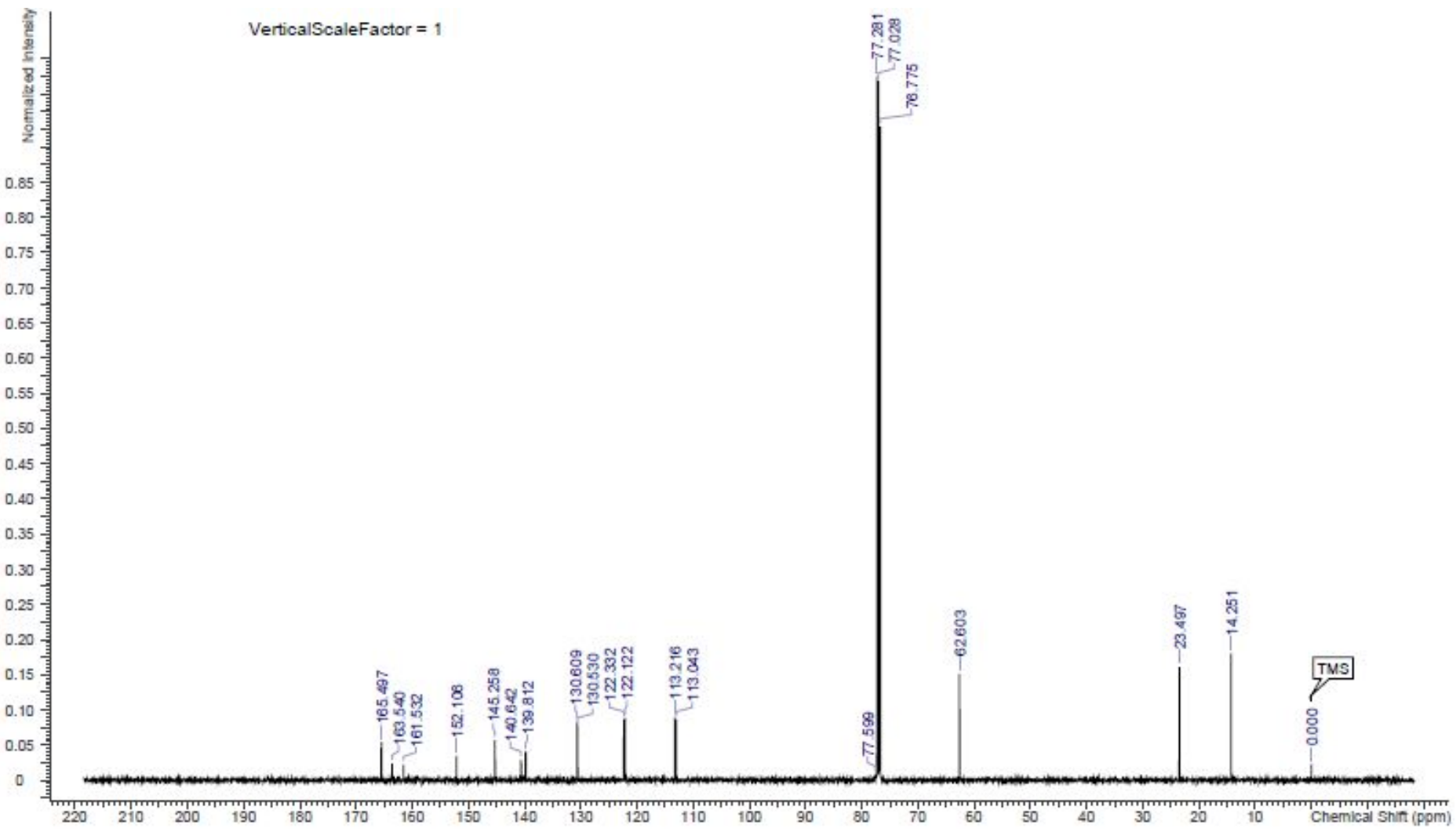


Compound 8

${ }^{1} \mathrm{H}$ NMR (DMSO-d ${ }_{6}$ )

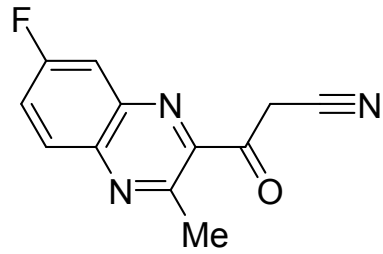

8
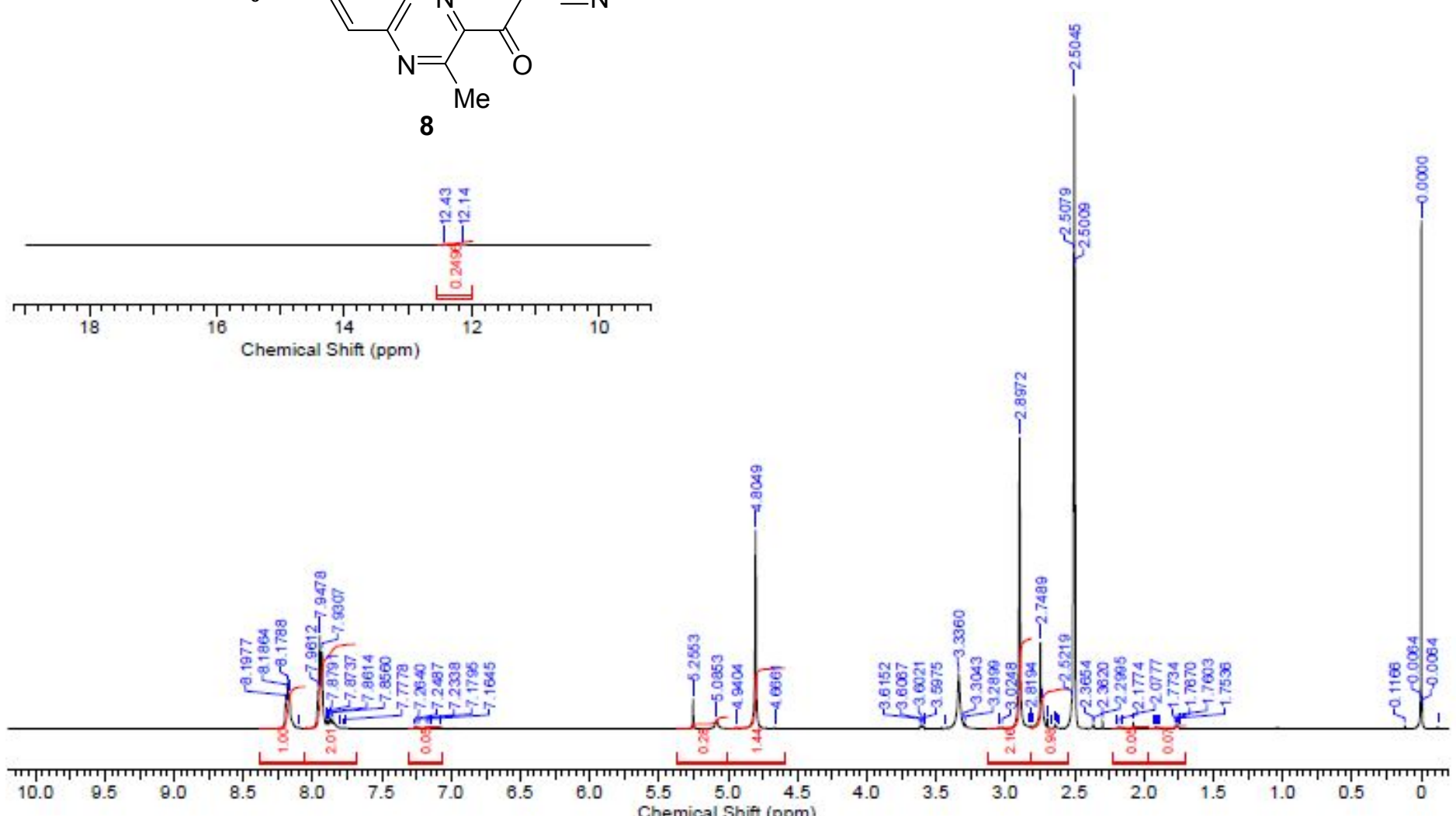

${ }^{13} \mathrm{C}$ NMR (DMSO-d ${ }_{6}$ )

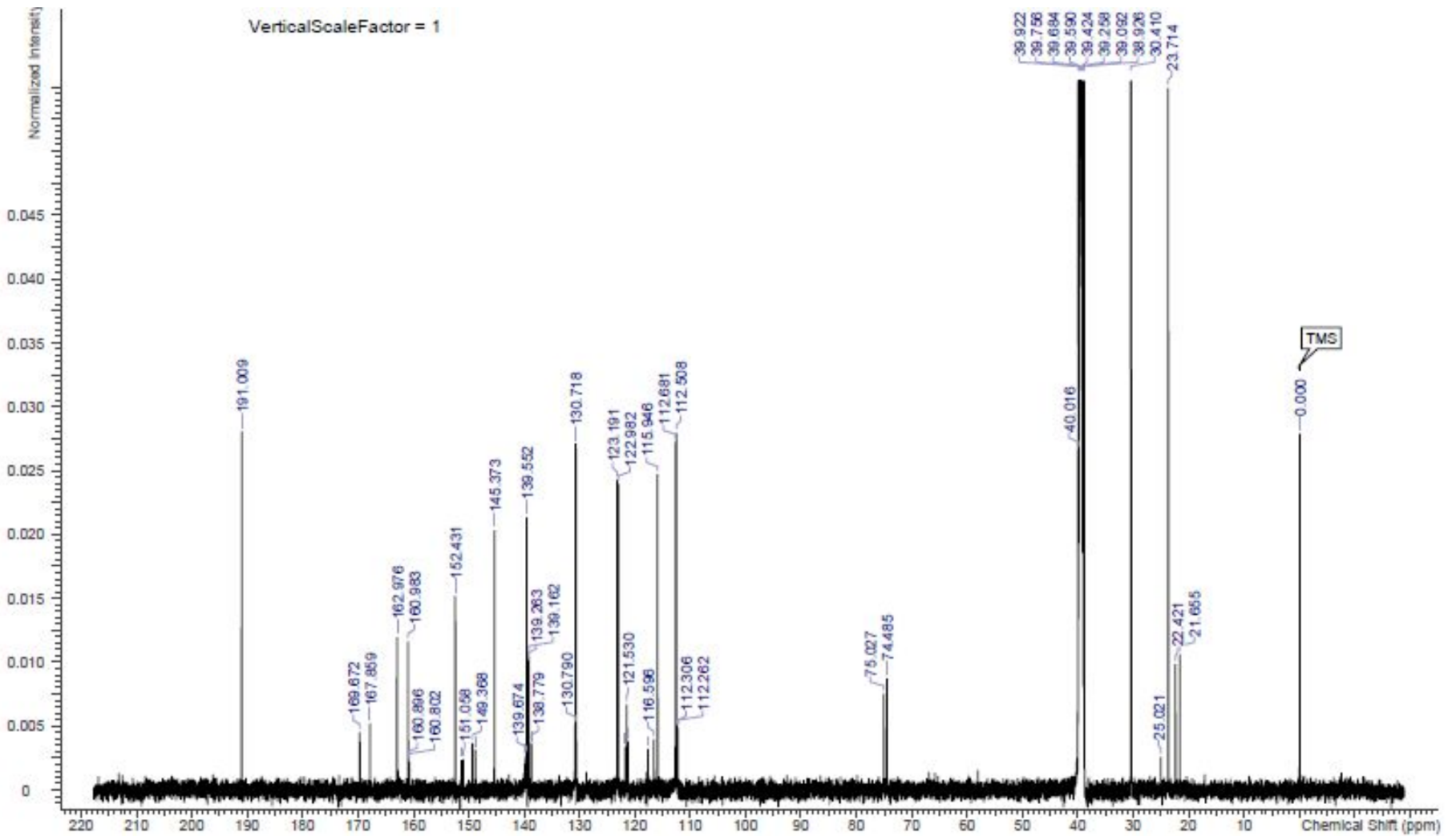


Compound 11

${ }^{1} \mathrm{H}$ NMR (DMSO-d ${ }_{6}$ )<smiles>Cc1nc2ccc(F)cc2nc1-c1cc2nc(O)cc(O)n2n1</smiles>
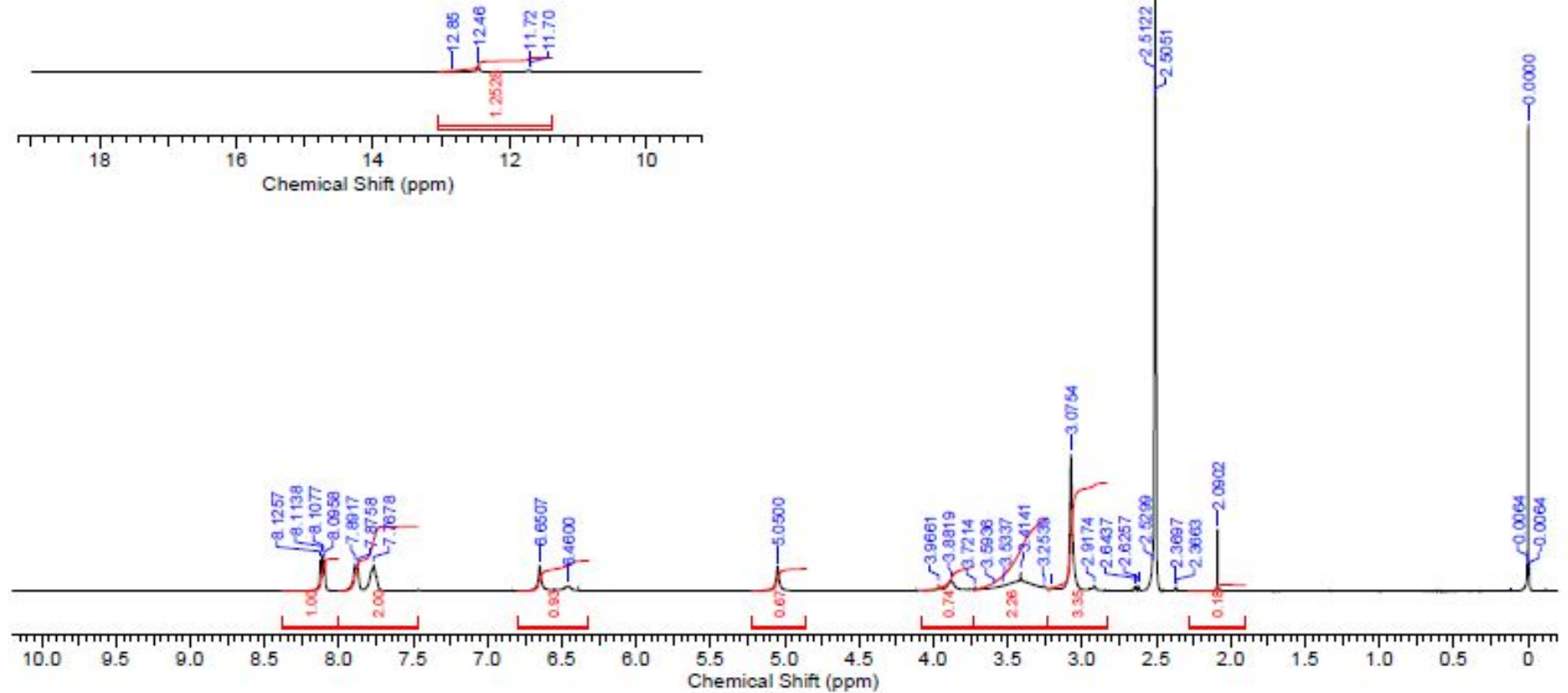

${ }^{13} \mathrm{C}$ NMR (DMSO-d ${ }_{6}$ )

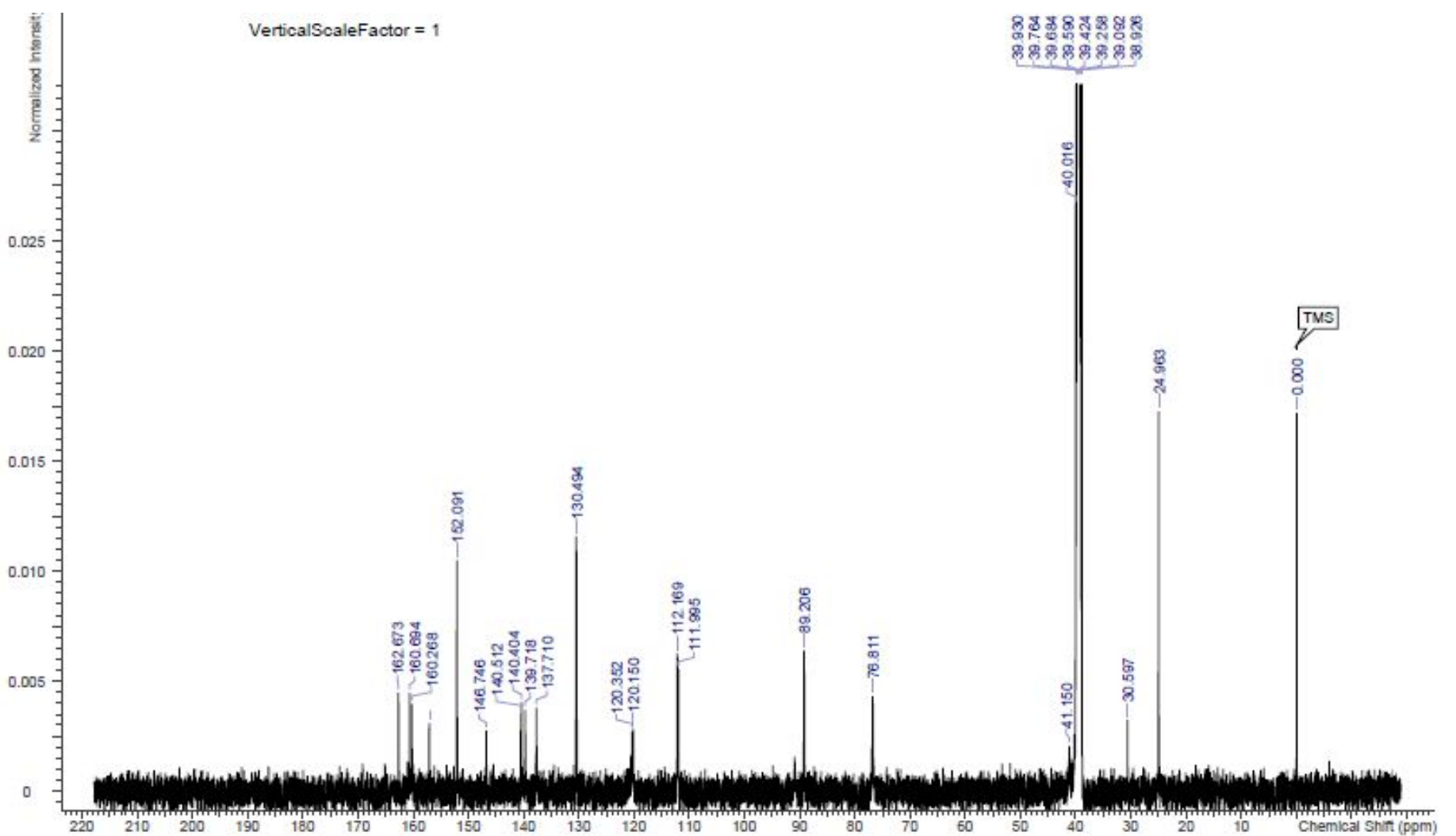




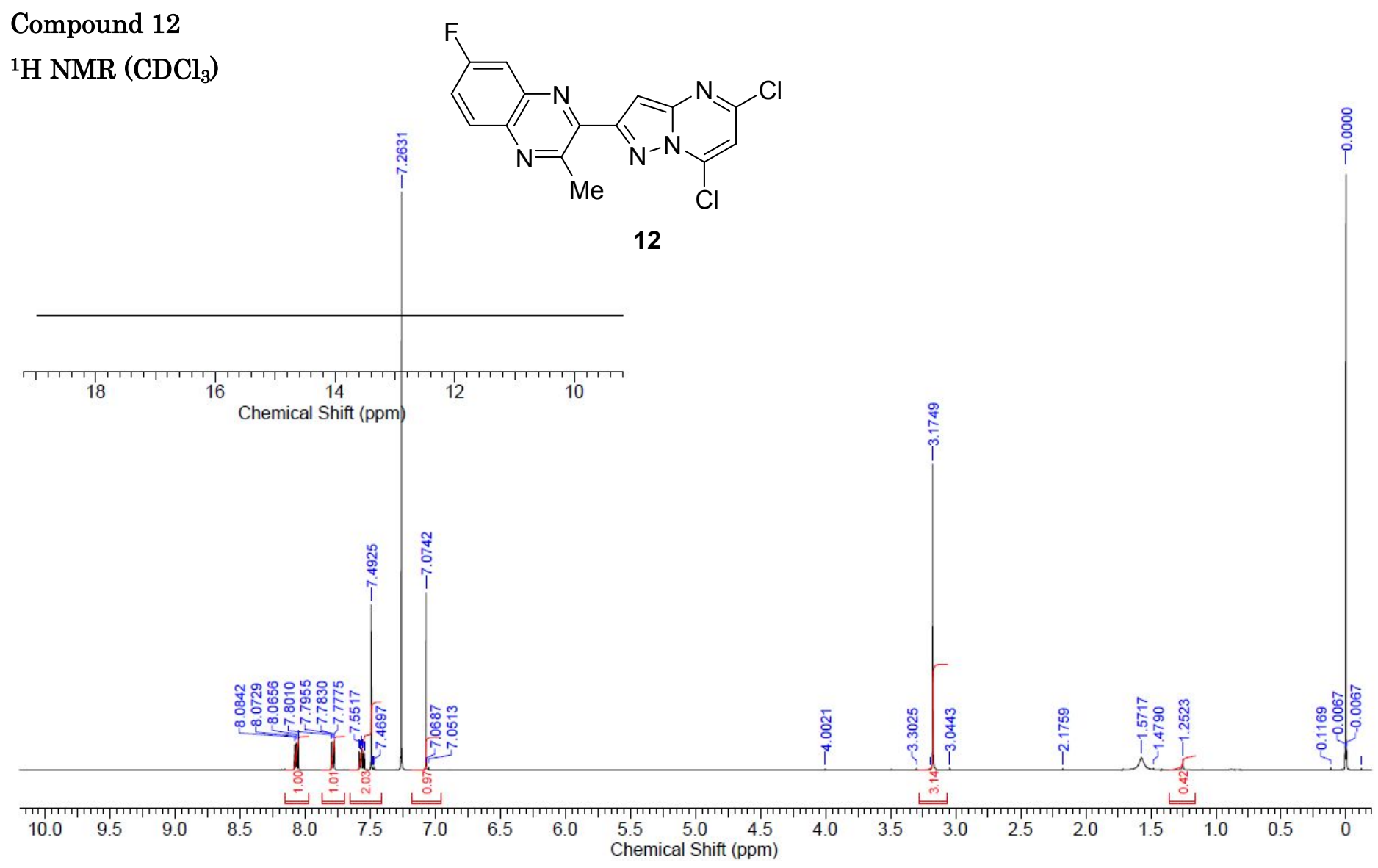

\section{${ }^{13} \mathrm{C} \mathrm{NMR}\left(\mathrm{CDCl}_{3}\right)$}

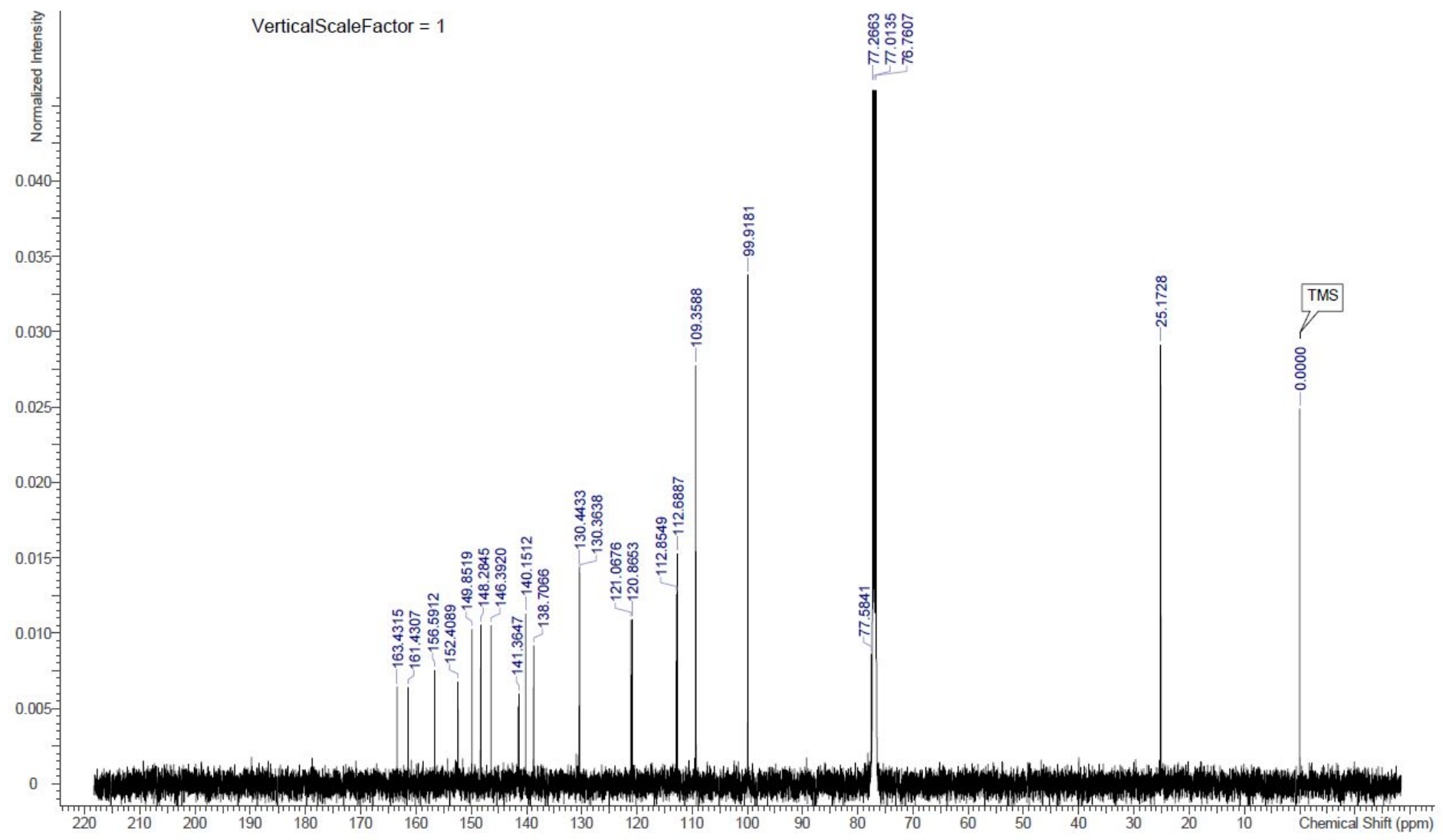


Compound 13

${ }^{1} \mathrm{H}$ NMR (DMSO-d $\mathrm{d}_{6}$ )<smiles>CNc1cc(Cl)nc2cc(-c3nc4cc(F)ccc4nc3C)nn12</smiles>

13

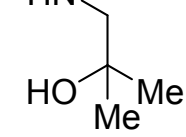

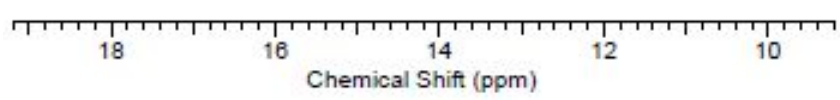

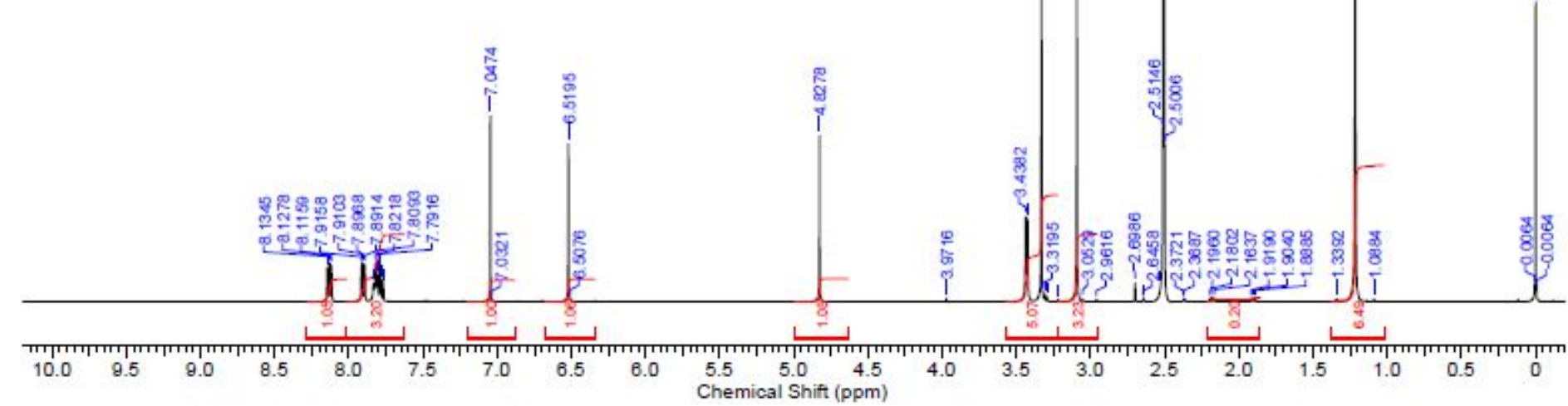

${ }^{13} \mathrm{C}$ NMR (DMSO-d ${ }_{6}$ )

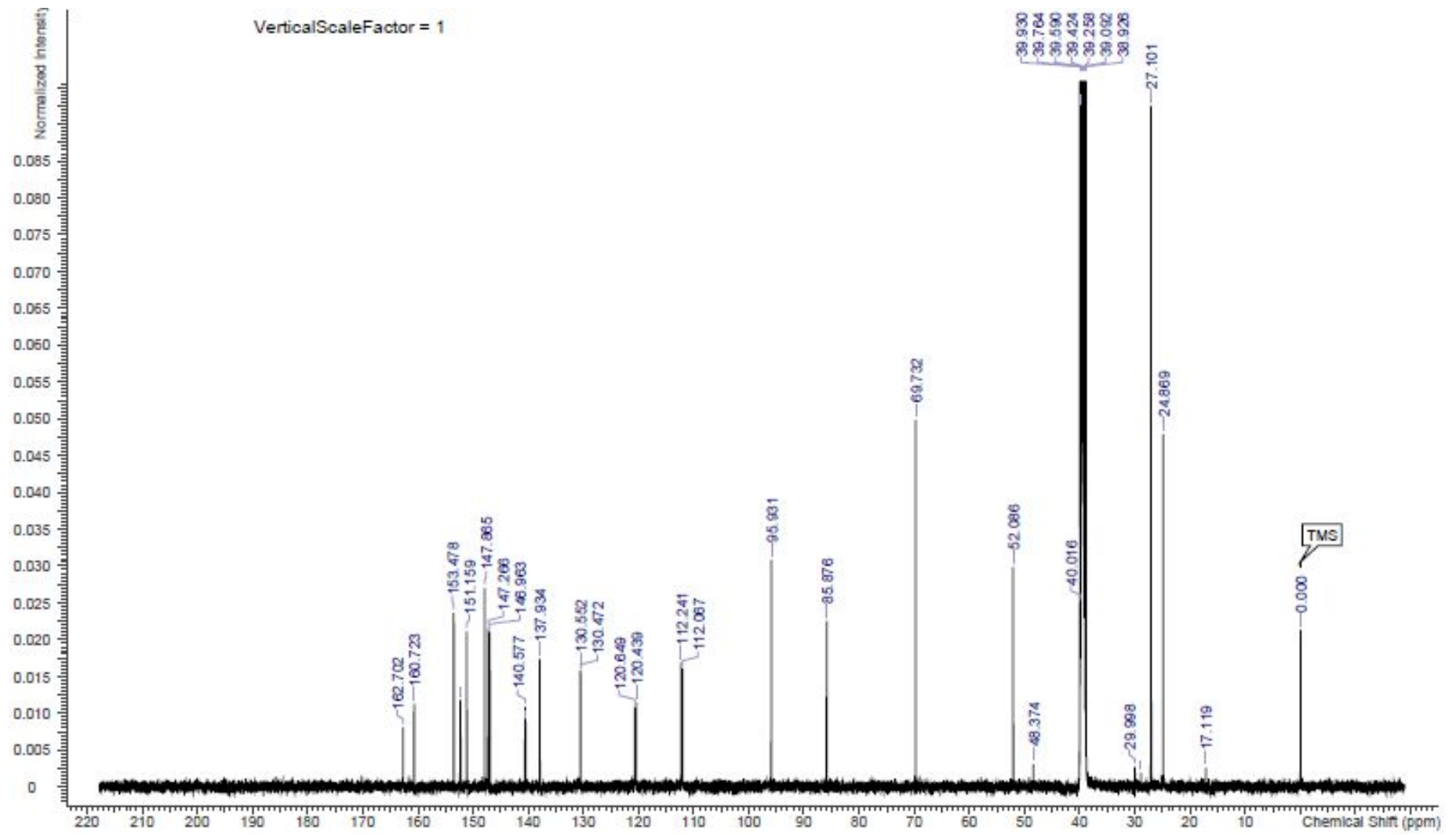


Compound 19

${ }^{1} \mathrm{H}$ NMR (DMSO-d ${ }_{6}$ )<smiles>Cc1nc2ccc(F)cc2nc1-c1cc(N)[nH]n1</smiles>

19
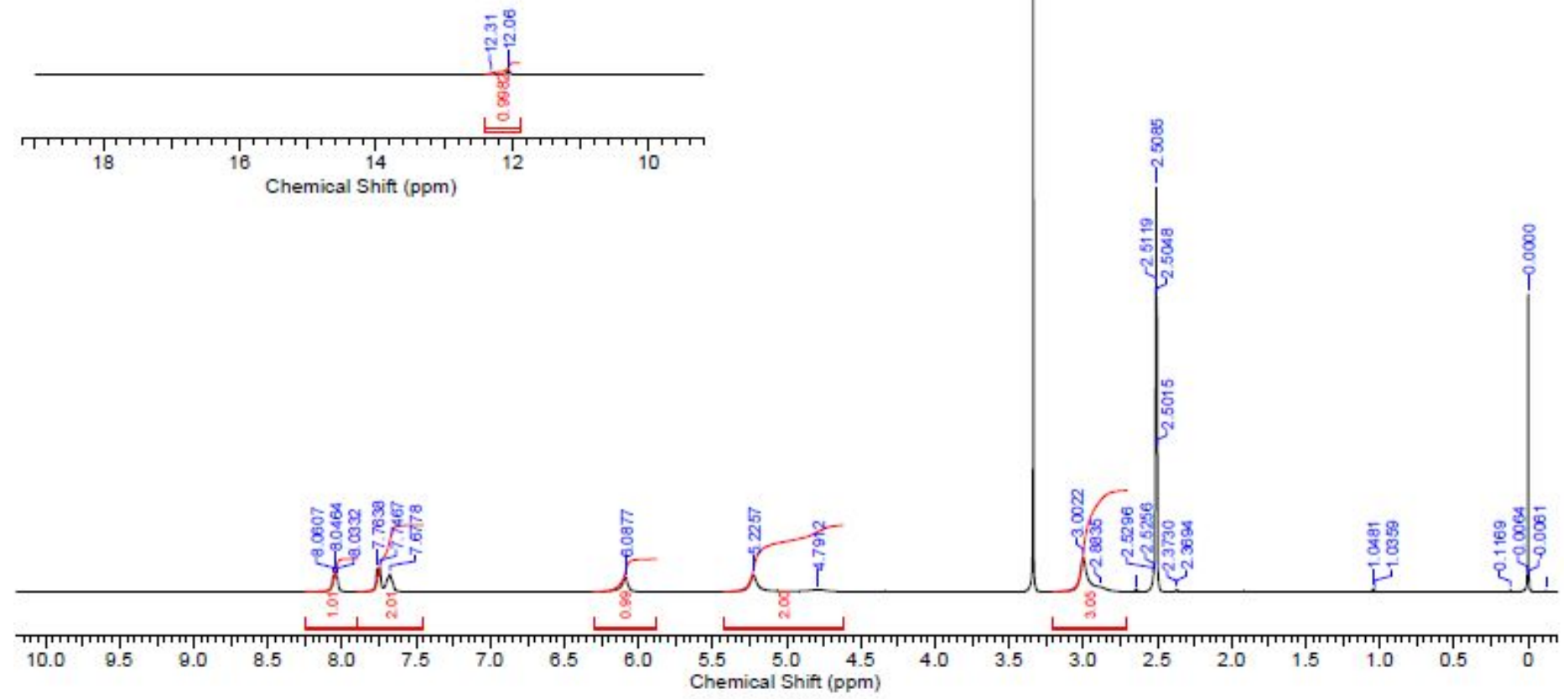

\section{${ }^{13} \mathrm{C}$ NMR (DMSO-d $\mathrm{d}_{6}$ )}

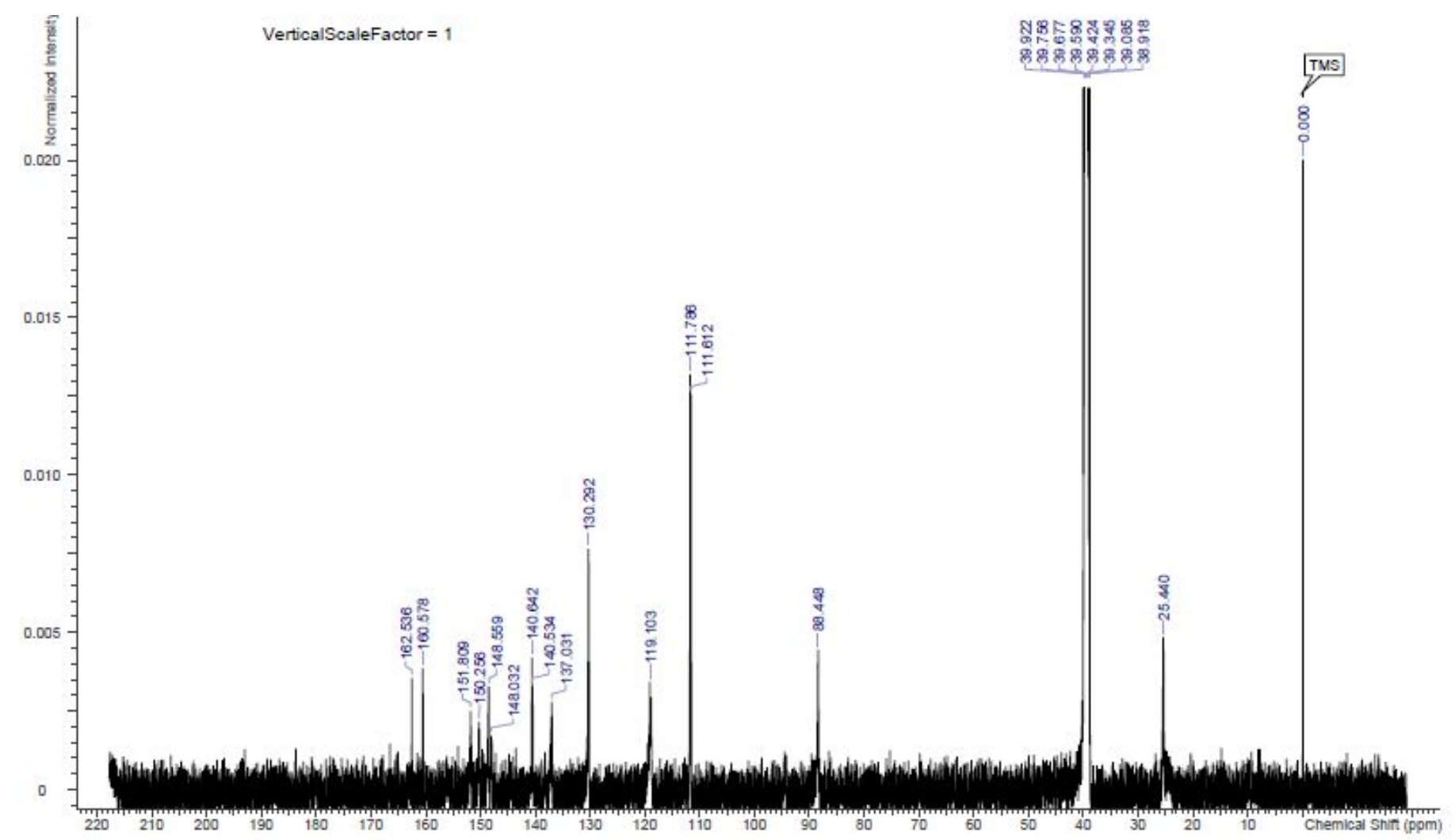


Compound 20

${ }^{1} \mathrm{H} \mathrm{NMR}\left(\mathrm{CDCl}_{3}\right)$

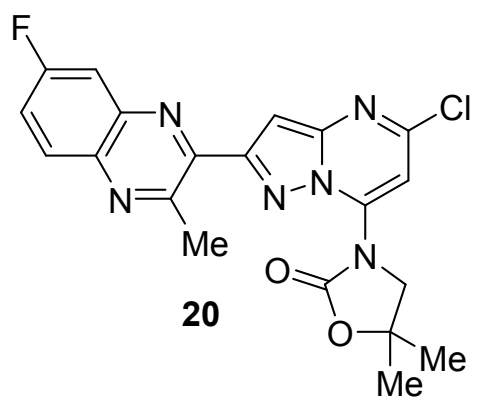

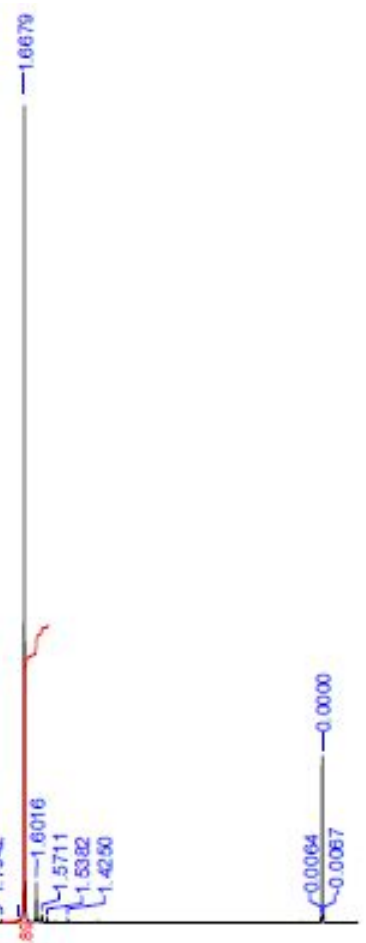

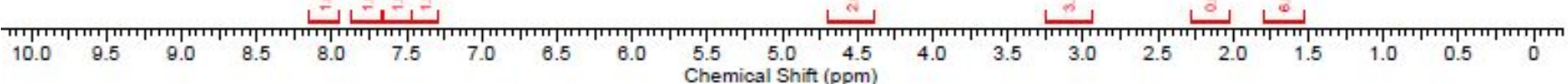

${ }^{13} \mathrm{C} \mathrm{NMR}\left(\mathrm{CDCl}_{3}\right)$

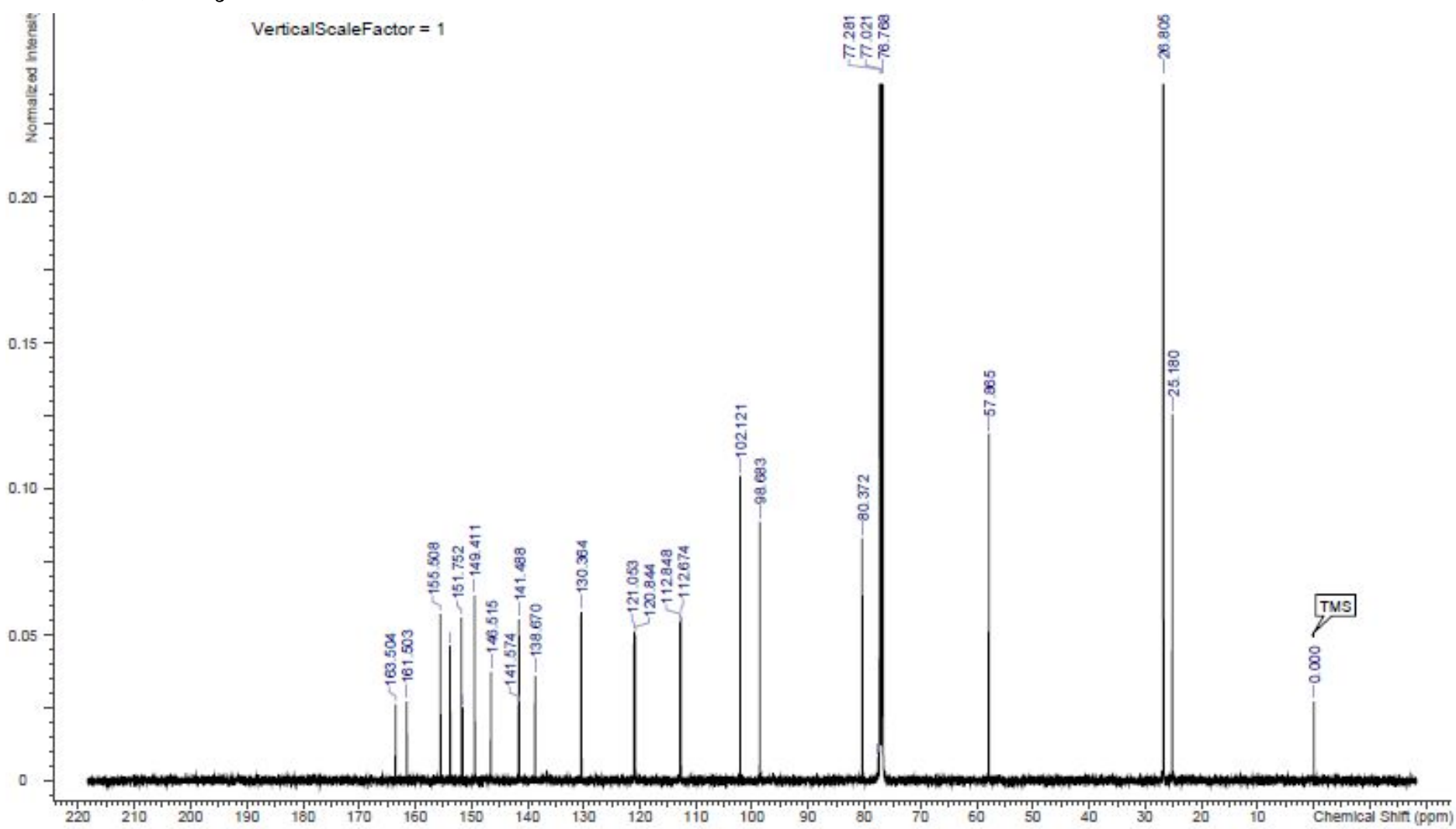


Compound 22

${ }^{1} \mathrm{H}$ NMR (DMSO-d ${ }_{6}$ )<smiles>CNc1nc2ccc(F)cc2nc1-c1cc2nc(N3CC[C@H](F)C3)cc(NC)n2n1</smiles>

22

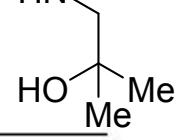

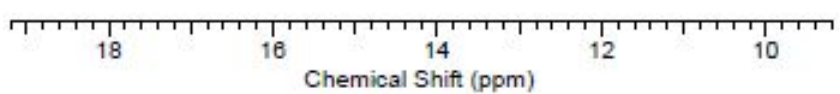

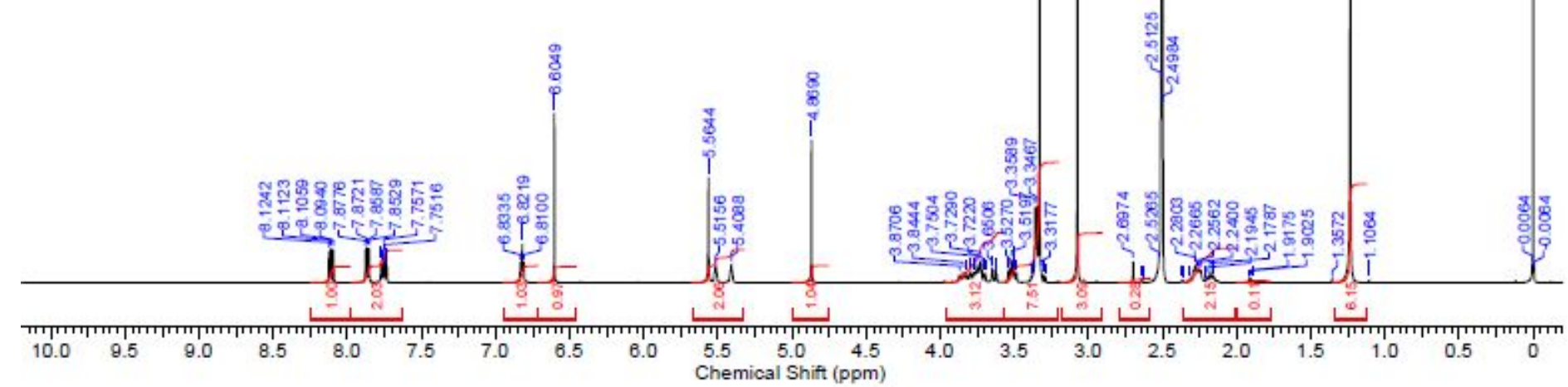

${ }^{13} \mathrm{C}$ NMR (DMSO-d $\mathrm{d}_{6}$ )

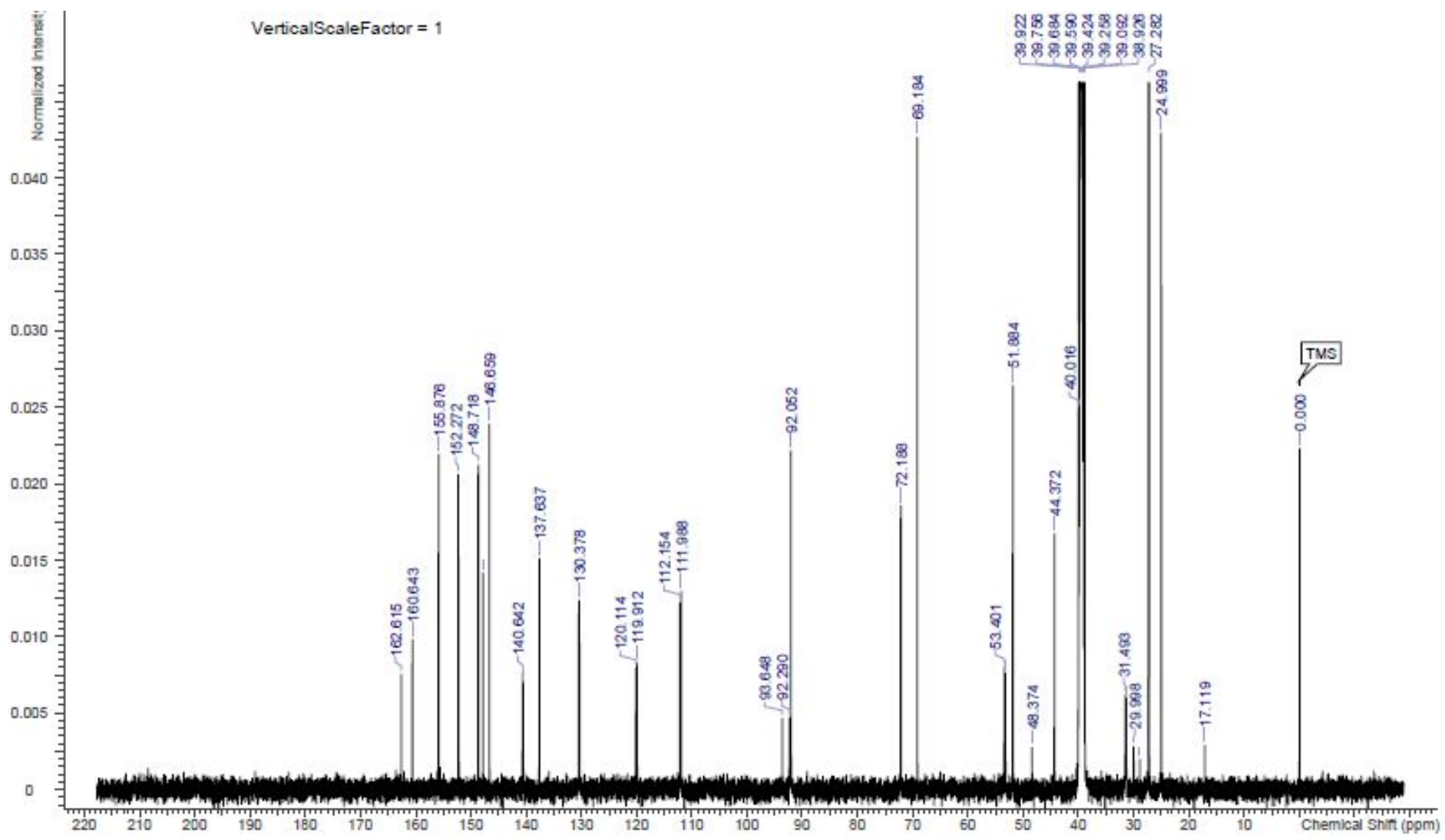




\section{X-ray Structure Report for Compound 1}

\section{Experimental for $\mathrm{X}$-ray structure}

\section{Data Collection}

A yellow block crystal of $\mathrm{C}_{23} \mathrm{H}_{26} \mathrm{ClF}_{2} \mathrm{~N}_{7} \mathrm{O}$ having approximate dimensions of $0.300 \times 0.100 \mathrm{x}$ $0.100 \mathrm{~mm}$ was mounted in a loop. All measurements were made on a Rigaku R-AXIS RAPID diffractometer using graphite monochromated $\mathrm{Cu}-\mathrm{K} \alpha$ radiation.

The crystal-to-detector distance was $127.40 \mathrm{~mm}$.

Cell constants and an orientation matrix for data collection corresponded to a primitive monoclinic cell with dimensions:

$$
\begin{aligned}
& a=15.866(3) \AA \\
& b=9.641(2) \AA \quad \beta=98.76(1)^{\circ} \\
& c=16.116(3) \AA \\
& V=2436.5(8) \AA^{3}
\end{aligned}
$$

For $Z=4$ and F.W. $=489.95$, the calculated density is $1.336 \mathrm{~g} / \mathrm{cm}^{3}$. Based on the reflection conditions of:

$$
\text { 0k0: } k=2 n
$$

packing considerations, a statistical analysis of intensity distribution, and the successful solution and refinement of the structure, the space group was determined to be:

$$
\text { P21 (\#4) }
$$

The data were collected at a temperature of $-20 \pm 1^{\circ} \mathrm{C}$ to a maximum $2 \theta$ value of $136.4^{\circ}$. A total of 54 oscillation images were collected. A sweep of data was done using $\omega$ scans from 50.0 to $230.0^{\circ}$ in $20.00^{\circ}$ step, at $\chi=50.0^{\circ}$ and $\phi=0.0^{\circ}$. The exposure rate was 30.0 [sec. $/ 0$ ]. A second sweep was performed using $\omega$ scans from 50.0 to $230.0^{\circ}$ in $10.00^{\circ}$ step, at $\chi=50.0^{\circ}$ and $\phi=90.0^{\circ}$. The exposure rate was 30.0 [sec./O]. Another sweep was performed using $\omega$ scans from 50.0 to 230.00 in $20.00^{\circ}$ step, at $\chi=50.0^{\circ}$ and $\phi=180.0^{\circ}$. The exposure rate was 30.0 [sec./ $/{ }^{\circ}$ ]. Another sweep was performed using $\omega$ scans from 50.0 to $230.0^{\circ}$ in $20.00^{\circ}$ step, at $\chi=50.0^{\circ}$ and $\phi=270.0^{\circ}$. The exposure rate was 30.0 [sec./O]. Another sweep was performed using $\omega$ scans from 50.0 to $230.0^{\circ}$ in $20.00^{\circ}$ 
step, at $\chi=10.00$ and $\phi=60.00$. The exposure rate was 30.0 [sec./0]. The crystal-to-detector distance was $127.40 \mathrm{~mm}$. Readout was performed in the $0.100 \mathrm{~mm}$ pixel mode.

\section{Data Reduction}

Of the 25465 reflections were collected, where 8389 were unique $\left(R_{\text {int }}=0.0327\right)$.

The linear absorption coefficient, $\mu$, for $\mathrm{Cu}-\mathrm{K} \alpha$ radiation is $17.795 \mathrm{~cm}^{-1}$. The data were corrected for Lorentz and polarization effects. A correction for secondary extinction ${ }^{1}$ was applied (coefficient $=0.000270)$.

\section{Structure Solution and Refinement}

The structure was solved by direct methods 2 and expanded using Fourier techniques. The non-hydrogen atoms were refined anisotropically. Hydrogen atoms were refined using the riding model. The final cycle of full-matrix least-squares refinement ${ }^{3}$ on $\mathrm{F}^{2}$ was based on 8389 observed reflections and 622 variable parameters and converged (largest parameter shift was 0.05 times its esd) with unweighted and weighted agreement factors of:

$$
\begin{gathered}
\mathrm{R} 1=\Sigma\|\mathrm{Fo}|-| \mathrm{Fc}\| / \Sigma|\mathrm{Fo}|=0.0392 \\
\mathrm{wR} 2=\left[\Sigma\left(\mathrm{w}\left(\mathrm{Fo}^{2}-\mathrm{Fc}^{2}\right)^{2}\right) / \Sigma \mathrm{w}\left(\mathrm{Fo}^{2}\right)^{2}\right]^{1 / 2}=0.1040
\end{gathered}
$$

The goodness of fit ${ }^{4}$ was 0.98 . Unit weights were used. The maximum and minimum peaks on the final difference Fourier map corresponded to 0.31 and $-0.21 \mathrm{e}^{-/ \AA^{3}}$, respectively. The final Flack parameter 5 was $0.027(8)$,

Neutral atom scattering factors were taken from International Tables for Crystallography (IT), Vol. C, Table 6.1.1.4 6. Anomalous dispersion effects were included in Fcalc 7 ; the values for $\Delta \mathrm{f}^{\prime}$ and $\Delta f^{\prime \prime}$ were those of Creagh and McAuley ${ }^{8}$. The values for the mass attenuation coefficients are those of Creagh and Hubbell 9 . All calculations were performed using the CrystalStructure 10 crystallographic software package except for refinement, which was performed using SHELXL Version 2016/611. 
References

(1) Larson, A.C. (1970), Crystallographic Computing, 291-294. F.R. Ahmed, ed. Munksgaard, Copenhagen (equation 22, with $\vee$ replaced by the cell volume).

(2) SHELXS97: Sheldrick, G. M. (2008). Acta Cryst. A64, 112-122.

(3) Least Squares function minimized: (SHELXL Version 2016/6)

$$
\Sigma w\left(\mathrm{~F}_{\mathrm{o}}^{2}-\mathrm{F}_{\mathrm{c}}{ }^{2}\right)^{2} \quad \text { where } \mathrm{w}=\text { Least Squares weights. }
$$

(4) Goodness of fit is defined as:

$$
\begin{aligned}
& {\left[\Sigma w\left(\mathrm{~F}_{\mathrm{o}}{ }^{2}-\mathrm{F}_{\mathrm{c}}{ }^{2}\right)^{2} /\left(\mathrm{N}_{\mathrm{o}}-\mathrm{N}_{\mathrm{v}}\right)\right]^{1 / 2}} \\
& \text { where: } \mathrm{N}_{\mathrm{O}}=\text { number of observations } \\
& \mathrm{N}_{\mathrm{V}} \text { = number of variables }
\end{aligned}
$$

(5) Parsons, S. and Flack, H. (2004), Acta Cryst. A60, s61.

(6) International Tables for Crystallography, Vol.C (1992). Ed. A.J.C. Wilson, Kluwer Academic Publishers, Dordrecht, Netherlands, Table 6.1.1.4, pp. 572.

(7) Ibers, J. A. \& Hamilton, W. C.; Acta Crystallogr., 17, 781 (1964).

(8) Creagh, D. C. \& McAuley, W.J .; "International Tables for Crystallography", Vol C, (A.J.C. Wilson, ed.), Kluwer Academic Publishers, Boston, Table 4.2.6.8, pages 219-222 (1992).

(9) Creagh, D. C. \& Hubbell, J.H..; "International Tables for Crystallography", Vol C, (A.J.C. Wilson, ed.), Kluwer Academic Publishers, Boston, Table 4.2.4.3, pages 200-206 (1992).

(10) CrystalStructure 4.2: Crystal Structure Analysis Package, Rigaku Corporation (2000-2017). Tokyo 196-8666, Japan.

(11) SHELXL Version 2016/6: Sheldrick, G. M. (2008). Acta Cryst. A64, 112-122. 


\author{
A. Crystal Data
}

Empirical Formula

$\mathrm{C}_{23} \mathrm{H}_{26} \mathrm{ClF}_{2} \mathrm{~N}_{7} \mathrm{O}$

Formula Weight

489.95

Crystal Color, Habit

yellow, block

Crystal Dimensions

$0.300 \times 0.100 \times 0.100 \mathrm{~mm}$

Crystal System

monoclinic

Lattice Type

Primitive

Lattice Parameters

$$
\begin{aligned}
& a=15.866(3) \AA \\
& b=9.641(2) \AA \\
& c=16.116(3) \AA \\
& \beta=98.76(1) \circ \\
& V=2436.5(8) \AA^{3}
\end{aligned}
$$

Space Group

$\mathrm{P} 21(\# 4)$

$Z$ value

4

$D_{\text {calc }}$

$1.336 \mathrm{~g} / \mathrm{cm}^{3}$

$F_{000}$

1024.00

$\mu(\mathrm{CuK} \alpha)$

$17.795 \mathrm{~cm}^{-1}$ 
B. Intensity Measurements

Diffractometer

Radiation

Voltage, Current

Temperature

Detector Aperture

Data Images

$\omega$ oscillation Range $(\chi=50.0, \phi=0.0)$

Exposure Rate

$\omega$ oscillation Range $(\chi=50.0, \phi=90.0)$

Exposure Rate

$\omega$ oscillation Range $(\chi=50.0, \phi=180.0)$

Exposure Rate

$\omega$ oscillation Range $(\chi=50.0, \phi=270.0)$

Exposure Rate

$\omega$ oscillation Range $(\chi=10.0, \phi=60.0)$

Exposure Rate

Detector Position
R-AXIS RAPID

$\operatorname{CuK} \alpha(\lambda=1.54187 \AA)$

graphite monochromated

$40 \mathrm{kV}, 100 \mathrm{~mA}$

$-20.0^{\circ} \mathrm{C}$

$460.0 \times 256.0 \mathrm{~mm}$

54 exposures

$50.0-230.00$

$30.0 \mathrm{sec} . / \mathrm{O}$

$50.0-230.0^{0}$

30.0 sec. $/ 0$

$50.0-230.00$

$30.0 \mathrm{sec} . / 0$

$50.0-230.00$

$30.0 \mathrm{sec} . / 0$

$50.0-230.00$

$30.0 \mathrm{sec} . / 0$

$127.40 \mathrm{~mm}$ 
Pixel Size

$2 \theta_{\max }$

No. of Reflections Measured

Corrections
$0.100 \mathrm{~mm}$

$136.4^{\circ}$

Total: 25465

Unique: $8389\left(R_{\text {int }}=0.0327\right)$

Parsons quotients (Flack x parameter): 2259

Lorentz-polarization

Secondary Extinction

(coefficient: 2.70000e-004) 


\section{Structure Solution and Refinement}

Structure Solution

Refinement

Function Minimized

Least Squares Weights

$2 \theta_{\max }$ cutoff

Anomalous Dispersion

No. Observations (All reflections)

No. Variables

Reflection/Parameter Ratio

Residuals: R1 (I>2.00\%(I))

Residuals: $\mathrm{R}$ (All reflections)

Residuals: wR2 (All reflections)

Goodness of Fit Indicator

Flack parameter (Parsons' quotients $=2259) \quad 0.027(8)$

Max Shift/Error in Final Cycle

Maximum peak in Final Diff. Map

Minimum peak in Final Diff. Map
Direct Methods (SHELXS97)

Full-matrix least-squares on $\mathrm{F}^{2}$

$\Sigma w\left(F^{2}-F^{2}\right)^{2}$

$$
\begin{aligned}
& \mathrm{w}=1 /\left[\sigma^{2}\left(\mathrm{Fo}^{2}\right)+(0.0551 \cdot \mathrm{P})^{2}\right. \\
& +0.0000 \cdot \mathrm{P}] \\
& \text { where } \mathrm{P}=\left(\operatorname{Max}\left(\mathrm{Fo}^{2}, 0\right)+2 \mathrm{Fc}^{2}\right) / 3
\end{aligned}
$$

$136.4^{\circ}$

All non-hydrogen atoms

8389

622

13.49

0.0392

0.0530

0.1040

0.976

0.048

$0.31 e^{-/} / \AA^{3}$

$-0.21 e^{-/} / \AA^{3}$ 
Table S1. Atomic coordinates and $\mathrm{B}_{\text {iso }} / \mathrm{B}_{\text {eq }}$

$\begin{array}{lllll}\text { atom } & \mathrm{x} & \mathrm{y} & \mathrm{z} & \mathrm{B} \text { eq } \\ \mathrm{F} 1 & 1.27909(16) & 0.8268(4) & 0.21249(19) & 6.41(8) \\ \mathrm{C} 2 & 1.2101(3) & 0.7628(7) & 0.1686(3) & 4.45(13) \\ \mathrm{C} 3 & 1.2242(3) & 0.6711(7) & 0.1058(3) & 5.02(14) \\ \mathrm{C} 4 & 1.1572(3) & 0.6045(6) & 0.0611(3) & 4.63(12) \\ \mathrm{C} 5 & 1.0733(3) & 0.6278(6) & 0.0787(3) & 3.57(11) \\ \mathrm{N} 6 & 1.0061(2) & 0.5600(5) & 0.0338(2) & 3.95(8) \\ \text { C7 } & 0.9292(3) & 0.5812(6) & 0.0532(3) & 3.49(10) \\ \text { C8 } & 0.8571(3) & 0.5048(6) & 0.0040(3) & 5.47(14) \\ \text { C9 } & 0.9167(3) & 0.6811(5) & 0.1167(3) & 2.82(10) \\ \text { N10 } & 0.9819(2) & 0.7512(4) & 0.1598(2) & 2.95(8) \\ \text { C11 } & 1.0616(3) & 0.7230(6) & 0.1415(3) & 3.09(9) \\ \text { C12 } & 1.1315(3) & 0.7905(6) & 0.1876(3) & 3.75(11) \\ \text { C13 } & 0.8344(3) & 0.7186(5) & 0.1412(2) & 2.66(9) \\ \text { N14 } & 0.7642(2) & 0.6393(4) & 0.1209(2) & 2.91(8) \\ \text { N15 } & 0.7037(2) & 0.7070(4) & 0.1571(2) & 2.43(7) \\ \text { C16 } & 0.6204(3) & 0.6637(5) & 0.1526(2) & 2.36(9) \\ \text { C17 } & 0.5668(3) & 0.7495(5) & 0.1896(2) & 2.48(8) \\ \text { C18 } & 0.5984(3) & 0.8704(5) & 0.2320(2) & 2.53(9) \\ \text { N19 } & 0.6818(2) & 0.9046(4) & 0.2369(2) & 2.66(8) \\ \text { C20 } & 0.7355(3) & 0.8244(5) & 0.1987(2) & 2.43(9) \\ \text { C21 } & 0.8187(3) & 0.8333(6) & 0.1904(3) & 2.83(9) \\ \text { N22 } & 0.6015(2) & 0.5428(4) & 0.11539(19) & 2.65(7) \\ \text { C23 } & 0.5196(2) & 0.4728(5) & 0.1101(2) & 2.82(9) \\ \text { C24 } & 0.4549(3) & 0.5123(5) & 0.0337(3) & 2.82(10) \\ \text { O25 } & 0.42650(18) & 0.6501(3) & 0.04921(19) & 3.39(7) \\ \text { C26 } & 0.3799(3) & 0.4119(5) & 0.0270(3) & 4.38(12) \\ \text { C27 } & 0.4955(3) & 0.5114(6) & -0.0443(3) & 4.02(12) \\ \text { N28 } & 0.5477(2) & 0.9560(4) & 0.2681(2) & 2.77(8) \\ \text { C29 } & 0.4552(3) & 0.9405(6) & 0.2577(3) & 3.27(10) \\ \text { C30 } & 0.4239(3) & 1.0768(6) & 0.2869(3) & 5.20(13) \\ \text { C31 } & 0.4977(3) & 1.1347(7) & 0.3450(3) & 5.43(14) \\ \text { C32 } & 0.5757(3) & 1.0899(5) & 0.3057(3) & 3.97(12) \\ \text { F33 } & 0.40838(19) & 1.1657(3) & 0.2162(2) & 7.85(9) \\ \text { F34 } & 0.21864(16) & 0.4425(5) & 0.2898(2) & 7.12(9)\end{array}$




$\begin{array}{lllll}\text { C35 } & 0.2870(3) & 0.5145(7) & 0.3296(4) & 4.66(14) \\ \text { C36 } & 0.2703(3) & 0.6106(7) & 0.3889(3) & 5.08(14) \\ \text { C37 } & 0.3368(3) & 0.6863(7) & 0.4296(3) & 4.91(13) \\ \text { C38 } & 0.4208(3) & 0.6627(6) & 0.4129(3) & 3.59(10) \\ \text { N39 } & 0.4868(2) & 0.7420(5) & 0.4529(2) & 3.83(9) \\ \text { C40 } & 0.5647(3) & 0.7132(6) & 0.4376(3) & 3.61(11) \\ \text { C41 } & 0.5779(3) & 0.6054(5) & 0.3793(3) & 2.67(9) \\ \text { N42 } & 0.5147(2) & 0.5324(4) & 0.3395(2) & 2.99(8) \\ \text { C43 } & 0.4346(3) & 0.5595(6) & 0.3544(3) & 3.15(9) \\ \text { C44 } & 0.3650(3) & 0.4844(6) & 0.3109(3) & 3.86(12) \\ \text { C45 } & 0.6351(3) & 0.8017(6) & 0.4828(3) & 4.93(13) \\ \text { C46 } & 0.6624(3) & 0.5687(5) & 0.3570(2) & 2.64(9) \\ \text { N47 } & 0.7322(2) & 0.6468(4) & 0.37772(19) & 2.71(8) \\ \text { N48 } & 0.7926(2) & 0.5775(4) & 0.3419(2) & 2.60(8) \\ \text { C49 } & 0.8767(3) & 0.6214(5) & 0.3463(3) & 2.40(9) \\ \text { C50 } & 0.9300(3) & 0.5352(5) & 0.3099(2) & 2.53(9) \\ \text { C51 } & 0.8978(3) & 0.4154(5) & 0.2660(2) & 2.44(9) \\ \text { N52 } & 0.8147(2) & 0.3820(4) & 0.26158(19) & 2.51(7) \\ \text { C53 } & 0.7609(3) & 0.4597(5) & 0.3012(3) & 2.53(9) \\ \text { C54 } & 0.6771(3) & 0.4495(6) & 0.3100(2) & 2.77(9) \\ \text { N55 } & 0.8957(2) & 0.7404(4) & 0.38537(18) & 2.66(7) \\ \text { C56 } & 0.9785(2) & 0.8092(5) & 0.3918(2) & 2.74(9) \\ \text { C57 } & 1.0421(3) & 0.7698(5) & 0.4696(3) & 2.74(9) \\ \text { O58 } & 1.07065(18) & 0.6320(3) & 0.45349(18) & 3.40(7) \\ \text { C59 } & 1.0002(3) & 0.7703(6) & 0.5485(2) & 3.75(11) \\ \text { C60 } & 1.1168(3) & 0.8700(6) & 0.4773(3) & 3.92(11) \\ \text { N61 } & 0.9474(2) & 0.3330(5) & 0.2267(2) & 2.83(8) \\ \text { C62 } & 0.9214(3) & 0.1944(5) & 0.1943(3) & 3.53(11) \\ \text { C63 } & 1.0045(3) & 0.1254(5) & 0.1868(3) & 3.66(9) \\ \text { C64 } & 1.0626(3) & 0.2424(5) & 0.1719(3) & 4.05(10) \\ \text { C65 } & 1.0395(3) & 0.3574(6) & 0.2295(3) & 3.29(10) \\ \text { F66 } & 1.03713(16) & 0.0633(3) & 0.26355(16) & 5.21(6) \\ \text { C167 } & 0.69849(6) & 0.23822(14) & 0.11588(7) & 4.34(3) \\ \text { C168 } & 0.80165(6) & 1.04918(14) & 0.38050(7) & 4.18(3)\end{array}$

$B_{\text {eq }}=8 / 3 \pi^{2}\left(U_{11}\left(a a^{*}\right)^{2}+U_{22}\left(b b^{\star}\right)^{2}+U_{33}\left(c c^{\star}\right)^{2}+2 U_{12}\left(a a^{*} b b^{*}\right) \cos \gamma+2 U_{13}\left(a a^{*} c c^{*}\right) \cos \beta+2 U_{23}\left(b b^{*} c c^{*}\right) \cos \alpha\right)$ 
Table S2. Atomic coordinates and $\mathrm{B}_{\text {iso }}$ involving hydrogen atoms

$\begin{array}{lcccl}\text { atom } & x & y & z & B \\ \text { H3 } & 1.27923 & 0.65559 & 0.09460 & 6.025 \\ \text { H4 } & 1.16601 & 0.54325 & 0.01866 & 5.556 \\ \text { H8A } & 0.81546 & 0.56976 & -0.02209 & 6.566 \\ \text { H8B } & 0.83144 & 0.44498 & 0.04071 & 6.566 \\ \text { H8C } & 0.87784 & 0.45017 & -0.03845 & 6.566 \\ \text { H12 } & 1.12430 & 0.85259 & 0.23009 & 4.494 \\ \text { H17 } & 0.50945 & 0.72690 & 0.18629 & 2.974 \\ \text { H19 } & 0.70103 & 0.97729 & 0.26440 & 3.194 \\ \text { H21 } & 0.85746 & 0.90105 & 0.21256 & 3.398 \\ \text { H22 } & 0.64058 & 0.50266 & 0.09261 & 3.184 \\ \text { H23A } & 0.49535 & 0.49346 & 0.16037 & 3.379 \\ \text { H23B } & 0.52916 & 0.37343 & 0.10891 & 3.379 \\ \text { H25 } & 0.38762 & 0.67216 & 0.01202 & 4.074 \\ \text { H26A } & 0.35684 & 0.41226 & 0.07867 & 5.258 \\ \text { H26B } & 0.39903 & 0.32015 & 0.01614 & 5.258 \\ \text { H26C } & 0.33669 & 0.44025 & -0.01815 & 5.258 \\ \text { H27A } & 0.45467 & 0.54114 & -0.09111 & 4.820 \\ \text { H27B } & 0.51438 & 0.41924 & -0.05443 & 4.820 \\ \text { H27C } & 0.54342 & 0.57338 & -0.03738 & 4.820 \\ \text { H29A } & 0.43933 & 0.86454 & 0.29158 & 3.926 \\ \text { H29B } & 0.43200 & 0.92344 & 0.19937 & 3.926 \\ \text { H30 } & 0.37327 & 1.06549 & 0.31435 & 6.243 \\ \text { H31A } & 0.50006 & 1.09662 & 0.40104 & 6.519 \\ \text { H31B } & 0.49409 & 1.23501 & 0.34805 & 6.519 \\ \text { H32A } & 0.58787 & 1.15560 & 0.26346 & 4.760 \\ \text { H32B } & 0.62584 & 1.07970 & 0.34794 & 4.760 \\ \text { H36 } & 0.21526 & 0.62334 & 0.40060 & 6.098 \\ \text { H37 } & 0.32699 & 0.75348 & 0.46846 & 5.891 \\ \text { H44 } & 0.37265 & 0.41726 & 0.27128 & 4.631 \\ \text { H45A } & 0.67430 & 0.74449 & 0.51905 & 5.918 \\ \text { H45B } & 0.61136 & 0.87043 & 0.51557 & 5.918 \\ \text { H45C } & 0.66473 & 0.84671 & 0.44255 & 5.918 \\ \text { H50 } & 0.98769 & 0.55630 & 0.31436 & 3.039 \\ \text { H52 } & 0.79515 & 0.31031 & 0.23319 & 3.016\end{array}$




$\begin{array}{lllll}\text { H54 } & 0.63848 & 0.38031 & 0.28983 & 3.323 \\ \text { H55 } & 0.85670 & 0.78008 & 0.40857 & 3.191 \\ \text { H56A } & 0.96957 & 0.90869 & 0.39213 & 3.293 \\ \text { H56B } & 1.00359 & 0.78694 & 0.34217 & 3.293 \\ \text { H58 } & 1.09693 & 0.59931 & 0.49685 & 4.085 \\ \text { H59A } & 1.04191 & 0.74841 & 0.59628 & 4.501 \\ \text { H59B } & 0.95541 & 0.70236 & 0.54307 & 4.501 \\ \text { H59C } & 0.97666 & 0.86044 & 0.55586 & 4.501 \\ \text { H60A } & 1.13872 & 0.87362 & 0.42504 & 4.701 \\ \text { H60B } & 1.16085 & 0.83897 & 0.52092 & 4.701 \\ \text { H60C } & 1.09811 & 0.96080 & 0.49093 & 4.701 \\ \text { H62A } & 0.88537 & 0.20041 & 0.14009 & 4.234 \\ \text { H62B } & 0.89118 & 0.14464 & 0.23298 & 4.234 \\ \text { H63 } & 0.99843 & 0.05815 & 0.14064 & 4.392 \\ \text { H64A } & 1.05241 & 0.27157 & 0.11362 & 4.856 \\ \text { H64B } & 1.12189 & 0.21565 & 0.18658 & 4.856 \\ \text { H65A } & 1.07025 & 0.34758 & 0.28597 & 3.950 \\ \text { H65B } & 1.05066 & 0.44850 & 0.20795 & 3.950\end{array}$


Table S3. Anisotropic displacement parameters

\begin{tabular}{|c|c|c|c|c|c|c|}
\hline atom & $U_{11}$ & $U_{22}$ & $\mathrm{U}_{33}$ & $U_{12}$ & $U_{13}$ & $U_{23}$ \\
\hline F1 & $0.0316(15)$ & $0.106(3)$ & $0.105(2)$ & $-0.0089(19)$ & $0.0073(16)$ & $0.006(2)$ \\
\hline $\mathrm{C} 2$ & $0.030(3)$ & $0.069(5)$ & $0.069(4)$ & $-0.002(3)$ & $0.006(3)$ & $0.013(4)$ \\
\hline C3 & $0.034(3)$ & $0.075(5)$ & $0.086(4)$ & $0.013(3)$ & $0.021(3)$ & $0.012(4)$ \\
\hline $\mathrm{C} 4$ & $0.050(3)$ & $0.066(4)$ & $0.065(3)$ & $0.004(3)$ & $0.025(3)$ & $0.002(3)$ \\
\hline C5 & $0.040(3)$ & $0.052(4)$ & $0.048(3)$ & $0.002(3)$ & $0.020(2)$ & $0.003(3)$ \\
\hline N6 & $0.050(2)$ & $0.056(3)$ & $0.049(2)$ & $0.007(2)$ & $0.0212(19)$ & $-0.005(2)$ \\
\hline $\mathrm{C7}$ & $0.045(3)$ & $0.048(4)$ & $0.041(2)$ & $-0.003(3)$ & $0.013(2)$ & $-0.006(2)$ \\
\hline $\mathrm{C} 8$ & $0.066(3)$ & $0.078(5)$ & $0.066(3)$ & $-0.010(3)$ & $0.017(3)$ & $-0.036(3)$ \\
\hline C9 & $0.033(2)$ & $0.040(3)$ & $0.035(2)$ & $0.001(2)$ & $0.009(2)$ & $0.002(2)$ \\
\hline N10 & $0.033(2)$ & $0.042(3)$ & $0.037(2)$ & $0.002(2)$ & $0.0062(16)$ & $0.007(2)$ \\
\hline C11 & $0.032(3)$ & $0.042(3)$ & $0.044(3)$ & $-0.003(3)$ & $0.010(2)$ & $0.009(3)$ \\
\hline $\mathrm{C} 12$ & $0.039(3)$ & $0.044(4)$ & $0.058(3)$ & $-0.005(3)$ & $0.007(2)$ & $0.006(3)$ \\
\hline $\mathrm{C} 13$ & $0.029(2)$ & $0.039(3)$ & $0.032(2)$ & $-0.002(3)$ & $0.0009(19)$ & $0.002(2)$ \\
\hline N14 & $0.030(2)$ & $0.041(3)$ & $0.039(2)$ & $0.002(2)$ & $0.0040(16)$ & $-0.000(2)$ \\
\hline N15 & $0.028(2)$ & $0.031(3)$ & $0.0332(19)$ & $-0.001(2)$ & $0.0030(15)$ & $-0.0055(19)$ \\
\hline C16 & $0.027(2)$ & $0.034(3)$ & $0.027(2)$ & $-0.002(2)$ & $-0.0017(18)$ & $-0.000(2)$ \\
\hline C17 & $0.026(2)$ & $0.032(3)$ & $0.036(2)$ & $-0.002(2)$ & $0.0013(19)$ & $0.000(2)$ \\
\hline C18 & $0.029(3)$ & $0.035(3)$ & $0.032(2)$ & $0.000(2)$ & $0.0024(19)$ & $0.001(2)$ \\
\hline N19 & $0.028(2)$ & $0.029(2)$ & $0.043(2)$ & $-0.0014(19)$ & $0.0029(16)$ & $-0.010(2)$ \\
\hline C20 & $0.027(2)$ & $0.034(3)$ & $0.030(2)$ & $0.001(3)$ & $0.0016(18)$ & $0.003(2)$ \\
\hline C21 & $0.029(2)$ & $0.035(3)$ & $0.042(3)$ & $-0.006(3)$ & $-0.000(2)$ & $-0.007(2)$ \\
\hline $\mathrm{N} 22$ & $0.025(2)$ & $0.034(3)$ & $0.040(2)$ & $0.001(2)$ & $0.0000(15)$ & $-0.007(2)$ \\
\hline $\mathrm{C} 23$ & $0.032(3)$ & $0.032(3)$ & $0.041(3)$ & $-0.001(2)$ & $0.000(2)$ & $-0.004(2)$ \\
\hline C24 & $0.035(3)$ & $0.034(3)$ & $0.036(3)$ & $0.002(2)$ & $-0.001(2)$ & $-0.007(2)$ \\
\hline $\mathrm{O} 25$ & $0.041(2)$ & $0.036(2)$ & $0.0469(19)$ & $0.0086(17)$ & $-0.0090(15)$ & $-0.0036(17)$ \\
\hline $\mathrm{C} 26$ & $0.045(3)$ & $0.041(4)$ & $0.073(3)$ & $-0.004(3)$ & $-0.017(3)$ & $-0.008(3)$ \\
\hline C27 & $0.056(3)$ & $0.050(4)$ & $0.044(3)$ & $0.007(3)$ & $-0.003(2)$ & $-0.009(3)$ \\
\hline N28 & $0.031(2)$ & $0.033(2)$ & $0.042(2)$ & $-0.002(2)$ & $0.0053(17)$ & $-0.008(2)$ \\
\hline C29 & $0.033(3)$ & $0.046(3)$ & $0.047(3)$ & $0.000(2)$ & $0.009(2)$ & $-0.003(2)$ \\
\hline C30 & $0.045(3)$ & $0.078(4)$ & $0.073(3)$ & $0.012(3)$ & $0.004(2)$ & $-0.024(3)$ \\
\hline C31 & $0.061(3)$ & $0.074(4)$ & $0.070(4)$ & $0.014(3)$ & $0.004(3)$ & $-0.031(3)$ \\
\hline C32 & $0.050(3)$ & $0.041(4)$ & $0.061(3)$ & $0.003(3)$ & $0.011(2)$ & $-0.020(3)$ \\
\hline F33 & $0.102(2)$ & $0.064(2)$ & $0.118(3)$ & $0.0269(19)$ & $-0.0263(19)$ & $-0.005(2)$ \\
\hline F34 & $0.0356(16)$ & $0.104(3)$ & $0.129(3)$ & $-0.015(2)$ & $0.0056(18)$ & $-0.013(3)$ \\
\hline
\end{tabular}




\begin{tabular}{|c|c|c|c|c|c|c|}
\hline C35 & $0.031(3)$ & $0.070(5)$ & $0.074(4)$ & $-0.000(3)$ & $0.003(3)$ & $0.016(4)$ \\
\hline C36 & $0.036(3)$ & $0.083(5)$ & $0.078(4)$ & $0.007(3)$ & $0.021(3)$ & $0.017(4)$ \\
\hline C37 & $0.047(3)$ & $0.081(5)$ & $0.062(3)$ & $0.021(3)$ & $0.022(3)$ & $0.004(3)$ \\
\hline C38 & $0.038(3)$ & $0.051(4)$ & $0.049(3)$ & $0.005(3)$ & $0.011(2)$ & $0.012(3)$ \\
\hline N39 & $0.047(2)$ & $0.056(3)$ & $0.045(2)$ & $0.005(2)$ & $0.0130(18)$ & $0.000(2)$ \\
\hline C40 & $0.042(3)$ & $0.057(4)$ & $0.039(2)$ & $0.003(3)$ & $0.010(2)$ & $-0.003(3)$ \\
\hline C41 & $0.031(2)$ & $0.037(3)$ & $0.034(2)$ & $0.003(2)$ & $0.0056(19)$ & $0.006(2)$ \\
\hline N42 & $0.030(2)$ & $0.041(3)$ & $0.043(2)$ & $-0.002(2)$ & $0.0054(17)$ & $0.000(2)$ \\
\hline C43 & $0.032(3)$ & $0.046(3)$ & $0.043(3)$ & $0.007(3)$ & $0.013(2)$ & $0.011(3)$ \\
\hline C44 & $0.029(3)$ & $0.065(4)$ & $0.053(3)$ & $-0.001(3)$ & $0.008(2)$ & $0.007(3)$ \\
\hline C45 & $0.053(3)$ & $0.076(4)$ & $0.060(3)$ & $-0.005(3)$ & $0.014(2)$ & $-0.029(3)$ \\
\hline C46 & $0.033(2)$ & $0.034(3)$ & $0.033(2)$ & $-0.001(3)$ & $0.0025(19)$ & $0.003(2)$ \\
\hline N47 & $0.032(2)$ & $0.035(3)$ & $0.036(2)$ & $0.003(2)$ & $0.0055(16)$ & $-0.005(2)$ \\
\hline N48 & $0.027(2)$ & $0.036(3)$ & $0.036(2)$ & $0.001(2)$ & $0.0036(16)$ & $-0.003(2)$ \\
\hline C49 & $0.031(2)$ & $0.029(3)$ & $0.030(2)$ & $-0.004(2)$ & $-0.0001(19)$ & $0.001(2)$ \\
\hline C50 & $0.028(2)$ & $0.034(3)$ & $0.033(2)$ & $-0.004(3)$ & $0.0012(19)$ & $-0.002(2)$ \\
\hline C51 & $0.034(3)$ & $0.031(3)$ & $0.027(2)$ & $0.002(2)$ & $0.0010(19)$ & $0.001(2)$ \\
\hline N52 & $0.028(2)$ & $0.030(3)$ & $0.037(2)$ & $-0.0037(19)$ & $0.0011(16)$ & $-0.0060(19)$ \\
\hline C53 & $0.033(3)$ & $0.027(3)$ & $0.035(3)$ & $-0.003(3)$ & $0.000(2)$ & $-0.008(2)$ \\
\hline C54 & $0.030(3)$ & $0.037(3)$ & $0.038(3)$ & $-0.002(3)$ & $0.004(2)$ & $-0.001(2)$ \\
\hline N55 & $0.031(2)$ & $0.033(3)$ & $0.036(2)$ & $-0.002(2)$ & $0.0018(15)$ & $-0.006(2)$ \\
\hline C56 & $0.032(2)$ & $0.031(3)$ & $0.040(3)$ & $-0.003(2)$ & $0.001(2)$ & $0.001(2)$ \\
\hline C57 & $0.035(3)$ & $0.027(3)$ & $0.039(3)$ & $-0.001(2)$ & $-0.004(2)$ & $-0.005(2)$ \\
\hline O58 & $0.046(2)$ & $0.037(2)$ & $0.0423(18)$ & $0.0091(18)$ & $-0.0063(15)$ & $-0.0055(17)$ \\
\hline C59 & $0.057(3)$ & $0.050(4)$ & $0.035(3)$ & $-0.003(3)$ & $0.006(2)$ & $-0.004(3)$ \\
\hline C60 & $0.038(3)$ & $0.053(4)$ & $0.056(3)$ & $-0.007(3)$ & $-0.000(2)$ & $-0.007(3)$ \\
\hline N61 & $0.030(2)$ & $0.033(2)$ & $0.043(2)$ & $0.002(2)$ & $0.0039(17)$ & $-0.006(2)$ \\
\hline C62 & $0.038(3)$ & $0.041(4)$ & $0.054(3)$ & $-0.003(3)$ & $0.004(2)$ & $-0.009(3)$ \\
\hline C63 & $0.045(3)$ & $0.044(3)$ & $0.050(3)$ & $0.004(2)$ & $0.009(2)$ & $-0.010(2)$ \\
\hline C64 & $0.039(2)$ & $0.053(3)$ & $0.065(3)$ & $0.003(2)$ & $0.016(2)$ & $-0.007(3)$ \\
\hline C65 & $0.030(2)$ & $0.045(3)$ & $0.051(3)$ & $-0.001(2)$ & $0.011(2)$ & $-0.001(2)$ \\
\hline F66 & $0.0697(17)$ & $0.0515(17)$ & $0.0771(18)$ & $0.0130(15)$ & $0.0125(14)$ & $0.0091(15)$ \\
\hline $\mathrm{Cl} 67$ & $0.0438(7)$ & $0.0551(10)$ & $0.0604(8)$ & $0.0011(7)$ & $-0.0095(6)$ & $-0.0198(8)$ \\
\hline $\mathrm{Cl} 68$ & $0.0417(7)$ & $0.0521(9)$ & $0.0600(8)$ & $-0.0003(7)$ & $-0.0088(6)$ & $-0.0159(7)$ \\
\hline
\end{tabular}

The general temperature factor expression: $\exp \left(-2 \pi^{2}\left(a^{*} 2 U_{11} h^{2}+b^{*} 2 U_{22} k^{2}+\left.c^{*} 2 U_{33}\right|^{2}+2 a^{*} b^{*} U_{12} h k\right.\right.$ $\left.\left.+2 a^{*} c^{*} U_{13} h l+2 b^{*} c^{*} U_{23} k l\right)\right)$ 
Table S4. Fragment Analysis

fragment: 1

$\begin{array}{lllll}\mathrm{F}(1) & \mathrm{C}(2) & \mathrm{C}(3) & \mathrm{C}(4) & \mathrm{C}(5) \\ \mathrm{N}(6) & \mathrm{C}(7) & \mathrm{C}(8) & \mathrm{C}(9) & \mathrm{N}(10) \\ \mathrm{C}(11) & \mathrm{C}(12) & \mathrm{C}(13) & \mathrm{N}(14) & \mathrm{N}(15) \\ \mathrm{C}(16) & \mathrm{C}(17) & \mathrm{C}(18) & \mathrm{N}(19) & \mathrm{C}(20) \\ \mathrm{C}(21) & \mathrm{N}(22) & \mathrm{C}(23) & \mathrm{C}(24) & \mathrm{O}(25) \\ \mathrm{C}(26) & \mathrm{C}(27) & \mathrm{N}(28) & \mathrm{C}(29) & \mathrm{C}(30) \\ \mathrm{C}(31) & \mathrm{C}(32) & \mathrm{F}(33) & & \end{array}$

fragment: 2

$\begin{array}{lllll}\mathrm{F}(34) & \mathrm{C}(35) & \mathrm{C}(36) & \mathrm{C}(37) & \mathrm{C}(38) \\ \mathrm{N}(39) & \mathrm{C}(40) & \mathrm{C}(41) & \mathrm{N}(42) & \mathrm{C}(43) \\ \mathrm{C}(44) & \mathrm{C}(45) & \mathrm{C}(46) & \mathrm{N}(47) & \mathrm{N}(48) \\ \mathrm{C}(49) & \mathrm{C}(50) & \mathrm{C}(51) & \mathrm{N}(52) & \mathrm{C}(53) \\ \mathrm{C}(54) & \mathrm{N}(55) & \mathrm{C}(56) & \mathrm{C}(57) & \mathrm{O}(58) \\ \mathrm{C}(59) & \mathrm{C}(60) & \mathrm{N}(61) & \mathrm{C}(62) & \mathrm{C}(63) \\ \mathrm{C}(64) & \mathrm{C}(65) & \mathrm{F}(66) & & \end{array}$

fragment: 3

$\mathrm{Cl}(67)$

fragment: 4

$\mathrm{Cl}(68)$ 
Table S5. Bond lengths ( $\AA$ )

\begin{tabular}{|c|c|c|c|c|c|}
\hline atom & atom & distance & atom & atom & distance \\
\hline F1 & $\mathrm{C} 2$ & $1.358(6)$ & $\mathrm{C} 2$ & $\mathrm{C} 3$ & $1.387(9)$ \\
\hline $\mathrm{C} 2$ & $\mathrm{C} 12$ & $1.355(7)$ & $\mathrm{C} 3$ & $\mathrm{C} 4$ & $1.353(7)$ \\
\hline $\mathrm{C} 4$ & C5 & $1.420(7)$ & C5 & N6 & $1.361(6)$ \\
\hline C5 & $\mathrm{C} 11$ & $1.399(7)$ & N6 & $\mathrm{C7}$ & $1.321(6)$ \\
\hline $\mathrm{C} 7$ & C8 & $1.485(7)$ & C7 & C9 & $1.440(7)$ \\
\hline C9 & N10 & $1.338(5)$ & C9 & C13 & $1.465(6)$ \\
\hline N10 & C11 & $1.369(6)$ & C11 & C12 & $1.398(6)$ \\
\hline $\mathrm{C} 13$ & N14 & $1.349(6)$ & $\mathrm{C} 13$ & $\mathrm{C} 21$ & $1.406(7)$ \\
\hline N14 & N15 & $1.364(5)$ & N15 & $\mathrm{C} 16$ & $1.378(5)$ \\
\hline N15 & $\mathrm{C} 20$ & $1.372(6)$ & C16 & $\mathrm{C} 17$ & $1.383(6)$ \\
\hline C16 & N22 & $1.324(6)$ & C17 & $\mathrm{C} 18$ & $1.405(6)$ \\
\hline C18 & N19 & $1.353(5)$ & C18 & N28 & $1.345(6)$ \\
\hline N19 & $\mathrm{C} 20$ & $1.365(6)$ & $\mathrm{C} 20$ & $\mathrm{C} 21$ & $1.350(6)$ \\
\hline N22 & $\mathrm{C} 23$ & $1.455(5)$ & C23 & C24 & $1.526(5)$ \\
\hline C24 & $\mathrm{O} 25$ & $1.437(6)$ & C24 & C26 & $1.525(6)$ \\
\hline $\mathrm{C} 24$ & $\mathrm{C} 27$ & $1.496(6)$ & N28 & $\mathrm{C} 29$ & $1.459(5)$ \\
\hline N28 & C32 & $1.466(6)$ & C29 & C30 & $1.506(8)$ \\
\hline C30 & C31 & $1.492(7)$ & C30 & F33 & $1.418(6)$ \\
\hline C31 & $\mathrm{C} 32$ & $1.537(7)$ & F34 & C35 & $1.363(6)$ \\
\hline C35 & C36 & $1.385(9)$ & $\mathrm{C} 35$ & C44 & $1.349(7)$ \\
\hline C36 & C37 & $1.366(7)$ & $\mathrm{C} 37$ & C38 & $1.418(7)$ \\
\hline C38 & N39 & $1.375(6)$ & C38 & C43 & $1.411(7)$ \\
\hline N39 & C40 & $1.327(6)$ & C40 & C41 & $1.438(7)$ \\
\hline C40 & C45 & $1.503(7)$ & C41 & N42 & $1.309(6)$ \\
\hline C41 & $\mathrm{C} 46$ & $1.482(6)$ & N42 & C43 & $1.354(6)$ \\
\hline C43 & C44 & $1.414(6)$ & C46 & N47 & $1.338(6)$ \\
\hline C46 & C54 & $1.415(7)$ & N47 & N48 & $1.366(5)$ \\
\hline N48 & $\mathrm{C} 49$ & $1.392(5)$ & N48 & C53 & $1.368(6)$ \\
\hline C49 & C50 & $1.378(6)$ & C49 & N55 & $1.321(6)$ \\
\hline C50 & C51 & $1.409(6)$ & C51 & N52 & $1.349(5)$ \\
\hline C51 & N61 & $1.344(6)$ & N52 & C53 & $1.365(6)$ \\
\hline C53 & C54 & $1.363(6)$ & N55 & C56 & $1.461(5)$ \\
\hline C56 & C57 & $1.533(5)$ & C57 & O58 & $1.441(6)$ \\
\hline C57 & C59 & $1.521(6)$ & C57 & C60 & $1.520(6)$ \\
\hline
\end{tabular}




$\begin{array}{llllll}\text { N61 } & \text { C62 } & 1.471(6) & \text { N61 } & \text { C65 } & 1.474(5) \\ \text { C62 } & \text { C63 } & 1.498(6) & \text { C63 } & \text { C64 } & 1.498(7) \\ \text { C63 } & \text { F66 } & 1.401(5) & \text { C64 } & \text { C65 } & 1.526(7)\end{array}$


Table S6. Bond lengths involving hydrogens $(\AA)$

\begin{tabular}{|c|c|c|c|c|c|}
\hline atom & atom & distance & atom & atom & distance \\
\hline C3 & H3 & 0.930 & C4 & $\mathrm{H} 4$ & 0.930 \\
\hline C8 & H8A & 0.960 & C8 & H8B & 0.960 \\
\hline C8 & $\mathrm{H} 8 \mathrm{C}$ & 0.960 & C12 & $\mathrm{H} 12$ & 0.930 \\
\hline C17 & $\mathrm{H} 17$ & 0.930 & N19 & H19 & 0.860 \\
\hline $\mathrm{C} 21$ & $\mathrm{H} 21$ & 0.930 & N22 & $\mathrm{H} 22$ & 0.860 \\
\hline $\mathrm{C} 23$ & $\mathrm{H} 23 \mathrm{~A}$ & 0.970 & $\mathrm{C} 23$ & $\mathrm{H} 23 \mathrm{~B}$ & 0.970 \\
\hline $\mathrm{O} 25$ & $\mathrm{H} 25$ & 0.820 & C26 & $\mathrm{H} 26 \mathrm{~A}$ & 0.960 \\
\hline C26 & H26B & 0.960 & C26 & $\mathrm{H} 26 \mathrm{C}$ & 0.960 \\
\hline $\mathrm{C} 27$ & $\mathrm{H} 27 \mathrm{~A}$ & 0.960 & $\mathrm{C} 27$ & H27B & 0.960 \\
\hline $\mathrm{C} 27$ & $\mathrm{H} 27 \mathrm{C}$ & 0.960 & C29 & $\mathrm{H} 29 \mathrm{~A}$ & 0.970 \\
\hline C29 & H29B & 0.970 & C30 & $\mathrm{H} 30$ & 0.980 \\
\hline C31 & $\mathrm{H} 31 \mathrm{~A}$ & 0.970 & C31 & H31B & 0.970 \\
\hline C32 & $\mathrm{H} 32 \mathrm{~A}$ & 0.970 & C32 & H32B & 0.970 \\
\hline C36 & H36 & 0.930 & C37 & $\mathrm{H} 37$ & 0.930 \\
\hline C44 & H44 & 0.930 & C45 & $\mathrm{H} 45 \mathrm{~A}$ & 0.960 \\
\hline C45 & $\mathrm{H} 45 \mathrm{~B}$ & 0.960 & C45 & $\mathrm{H} 45 \mathrm{C}$ & 0.960 \\
\hline C50 & H50 & 0.930 & N52 & H52 & 0.860 \\
\hline C54 & H54 & 0.930 & N55 & H55 & 0.860 \\
\hline C56 & $\mathrm{H} 56 \mathrm{~A}$ & 0.970 & C56 & H56B & 0.970 \\
\hline O58 & H58 & 0.820 & C59 & H59A & 0.960 \\
\hline C59 & H59B & 0.960 & C59 & H59C & 0.960 \\
\hline C60 & $\mathrm{H} 60 \mathrm{~A}$ & 0.960 & C60 & H60B & 0.960 \\
\hline C60 & $\mathrm{H} 60 \mathrm{C}$ & 0.960 & C62 & $\mathrm{H} 62 \mathrm{~A}$ & 0.970 \\
\hline C62 & H62B & 0.970 & C63 & $\mathrm{H} 63$ & 0.980 \\
\hline C64 & H64A & 0.970 & C64 & H64B & 0.970 \\
\hline C65 & H65A & 0.970 & C65 & H65B & 0.970 \\
\hline
\end{tabular}


Table S7. Bond angles ( $\left.{ }^{\circ}\right)$

\begin{tabular}{|c|c|c|c|c|c|c|c|}
\hline atom & atom & atom & angle & atom & atom & atom & angle \\
\hline $\mathrm{F} 1$ & C2 & C3 & $117.6(4)$ & F1 & $\mathrm{C} 2$ & $\mathrm{C} 12$ & $119.3(5)$ \\
\hline $\mathrm{C} 3$ & $\mathrm{C} 2$ & C12 & $123.2(5)$ & $\mathrm{C} 2$ & $\mathrm{C} 3$ & C4 & $119.3(5)$ \\
\hline C3 & C4 & C5 & $120.2(5)$ & C4 & C5 & N6 & $120.0(5)$ \\
\hline C4 & C5 & C11 & $118.7(4)$ & N6 & C5 & C11 & $121.4(4)$ \\
\hline C5 & N6 & $\mathrm{C} 7$ & 118.4(4) & N6 & C7 & C8 & $117.4(4)$ \\
\hline N6 & $\mathrm{C} 7$ & C9 & $120.3(4)$ & C8 & $\mathrm{C} 7$ & C9 & $122.2(4)$ \\
\hline $\mathrm{C} 7$ & C9 & N10 & 121.8(4) & C7 & C9 & C13 & $125.5(4)$ \\
\hline N10 & C9 & C13 & $112.7(4)$ & C9 & N10 & C11 & $117.0(4)$ \\
\hline C5 & C11 & N10 & $121.0(4)$ & C5 & C11 & $\mathrm{C} 12$ & $120.5(4)$ \\
\hline N10 & C11 & C12 & $118.5(4)$ & C2 & C12 & C11 & $118.1(5)$ \\
\hline C9 & C13 & N14 & $122.1(4)$ & C9 & C13 & $\mathrm{C} 21$ & $126.0(4)$ \\
\hline N14 & C13 & C21 & $111.8(4)$ & C13 & N14 & N15 & 103.6(4) \\
\hline N14 & N15 & C16 & $124.4(4)$ & N14 & N15 & $\mathrm{C} 20$ & $112.0(3)$ \\
\hline $\mathrm{C} 16$ & N15 & $\mathrm{C} 20$ & $123.7(4)$ & N15 & C16 & C17 & $116.3(4)$ \\
\hline N15 & C16 & N22 & 116.1(4) & C17 & C16 & N22 & $127.6(4)$ \\
\hline $\mathrm{C} 16$ & C17 & C18 & $120.6(4)$ & $\mathrm{C} 17$ & C18 & N19 & $120.2(4)$ \\
\hline $\mathrm{C} 17$ & C18 & N28 & $121.8(4)$ & N19 & C18 & N28 & $117.9(4)$ \\
\hline C18 & N19 & C20 & $120.5(4)$ & N15 & C20 & N19 & $118.6(4)$ \\
\hline N15 & C20 & C21 & $107.2(4)$ & N19 & $\mathrm{C} 20$ & $\mathrm{C} 21$ & $134.1(4)$ \\
\hline C13 & C21 & C20 & $105.4(4)$ & C16 & N22 & $\mathrm{C} 23$ & 125.1(4) \\
\hline N22 & $\mathrm{C} 23$ & $\mathrm{C} 24$ & $114.6(4)$ & $\mathrm{C} 23$ & $\mathrm{C} 24$ & $\mathrm{O} 25$ & $106.3(3)$ \\
\hline $\mathrm{C} 23$ & $\mathrm{C} 24$ & $\mathrm{C} 26$ & $108.7(4)$ & $\mathrm{C} 23$ & $\mathrm{C} 24$ & $\mathrm{C} 27$ & $110.7(4)$ \\
\hline $\mathrm{O} 25$ & $\mathrm{C} 24$ & $\mathrm{C} 26$ & $109.7(4)$ & $\mathrm{O} 25$ & $\mathrm{C} 24$ & $\mathrm{C} 27$ & $109.8(4)$ \\
\hline $\mathrm{C} 26$ & $\mathrm{C} 24$ & $\mathrm{C} 27$ & $111.6(4)$ & $\mathrm{C} 18$ & N28 & $\mathrm{C} 29$ & $123.0(4)$ \\
\hline C18 & N28 & C32 & $123.7(4)$ & $\mathrm{C} 29$ & N28 & C32 & $111.9(4)$ \\
\hline N28 & C29 & C30 & $104.4(4)$ & C29 & C30 & C31 & $104.9(4)$ \\
\hline $\mathrm{C} 29$ & C30 & F33 & $107.5(4)$ & C31 & C30 & F33 & $107.6(4)$ \\
\hline C30 & C31 & $\mathrm{C} 32$ & $103.9(4)$ & N28 & C32 & C31 & $101.9(4)$ \\
\hline F34 & C35 & C36 & $116.3(4)$ & F34 & C35 & C44 & $118.5(5)$ \\
\hline $\mathrm{C} 36$ & C35 & C44 & $125.1(5)$ & C35 & C36 & C37 & $118.3(5)$ \\
\hline C36 & C37 & $\mathrm{C} 38$ & $120.1(5)$ & $\mathrm{C} 37$ & C38 & N39 & $119.4(5)$ \\
\hline C37 & C38 & C43 & 119.1(4) & N39 & C38 & C43 & $121.4(4)$ \\
\hline C38 & N39 & C40 & $117.4(4)$ & N39 & C40 & C41 & $120.3(4)$ \\
\hline N39 & C40 & C45 & $115.9(5)$ & C41 & C40 & C45 & $123.8(4)$ \\
\hline
\end{tabular}




$\begin{array}{llllllll}\text { C40 } & \text { C41 } & \text { N42 } & 122.1(4) & \text { C40 } & \text { C41 } & \text { C46 } & 123.8(4) \\ \text { N42 } & \text { C41 } & \text { C46 } & 114.0(4) & \text { C41 } & \text { N42 } & \text { C43 } & 118.6(4) \\ \text { C38 } & \text { C43 } & \text { N42 } & 120.0(4) & \text { C38 } & \text { C43 } & \text { C44 } & 120.2(4) \\ \text { N42 } & \text { C43 } & \text { C44 } & 119.8(5) & \text { C35 } & \text { C44 } & \text { C43 } & 117.0(5) \\ \text { C41 } & \text { C46 } & \text { N47 } & 123.4(4) & \text { C41 } & \text { C46 } & \text { C54 } & 123.5(4) \\ \text { N47 } & \text { C46 } & \text { C54 } & 113.1(4) & \text { C46 } & \text { N47 } & \text { N48 } & 103.1(3) \\ \text { N47 } & \text { N48 } & \text { C49 } & 124.0(4) & \text { N47 } & \text { N48 } & \text { C53 } & 112.2(3) \\ \text { C49 } & \text { N48 } & \text { C53 } & 123.8(4) & \text { N48 } & \text { C49 } & \text { C50 } & 116.1(4) \\ \text { N48 } & \text { C49 } & \text { N55 } & 115.8(4) & \text { C50 } & \text { C49 } & \text { N55 } & 128.1(4) \\ \text { C49 } & \text { C50 } & \text { C51 } & 120.6(4) & \text { C50 } & \text { C51 } & \text { N52 } & 119.9(4) \\ \text { C50 } & \text { C51 } & \text { N61 } & 121.9(4) & \text { N52 } & \text { C51 } & \text { N61 } & 118.2(4) \\ \text { C51 } & \text { N52 } & \text { C53 } & 121.3(4) & \text { N48 } & \text { C53 } & \text { N52 } & 118.1(4) \\ \text { N48 } & \text { C53 } & \text { C54 } & 107.7(4) & \text { N52 } & \text { C53 } & \text { C54 } & 134.3(4) \\ \text { C46 } & \text { C54 } & \text { C53 } & 103.9(4) & \text { C49 } & \text { N55 } & \text { C56 } & 124.4(4) \\ \text { N55 } & \text { C56 } & \text { C57 } & 114.7(4) & \text { C56 } & \text { C57 } & \text { O58 } & 105.4(3) \\ \text { C56 } & \text { C57 } & \text { C59 } & 111.5(3) & \text { C56 } & \text { C57 } & \text { C60 } & 108.5(4) \\ \text { O58 } & \text { C57 } & \text { C59 } & 110.1(4) & \text { O58 } & \text { C57 } & \text { C60 } & 109.8(4) \\ \text { C59 } & \text { C57 } & \text { C60 } & 111.4(4) & \text { C51 } & \text { N61 } & \text { C62 } & 123.5(4) \\ \text { C51 } & \text { N61 } & \text { C65 } & 122.7(4) & \text { C62 } & \text { N61 } & \text { C65 } & 112.3(4) \\ \text { N61 } & \text { C62 } & \text { C63 } & 103.3(3) & \text { C62 } & \text { C63 } & \text { C64 } & 104.4(4) \\ \text { C62 } & \text { C63 } & \text { F66 } & 109.3(4) & \text { C64 } & \text { C63 } & \text { F66 } & 108.0(3) \\ \text { C63 } & \text { C64 } & \text { C65 } & 103.9(4) & \text { N61 } & \text { C65 } & \text { C64 } & 101.2(4)\end{array}$


Table S8. Bond angles involving hydrogens $\left({ }^{\circ}\right)$

\begin{tabular}{|c|c|c|c|c|c|c|c|}
\hline atom & atom & atom & angle & atom & atom & atom & angle \\
\hline $\mathrm{C} 2$ & $\mathrm{C} 3$ & $\mathrm{H} 3$ & 120.3 & $\mathrm{C} 4$ & C3 & $\mathrm{H} 3$ & 120.3 \\
\hline C3 & C4 & $\mathrm{H} 4$ & 119.9 & C5 & C4 & $\mathrm{H} 4$ & 119.9 \\
\hline $\mathrm{C7}$ & $\mathrm{C} 8$ & $\mathrm{H} 8 \mathrm{~A}$ & 109.5 & C7 & $\mathrm{C} 8$ & H8B & 109.5 \\
\hline $\mathrm{C} 7$ & C8 & $\mathrm{H} 8 \mathrm{C}$ & 109.5 & $\mathrm{H} 8 \mathrm{~A}$ & C8 & H8B & 109.5 \\
\hline $\mathrm{H} 8 \mathrm{~A}$ & C8 & $\mathrm{H} 8 \mathrm{C}$ & 109.5 & $\mathrm{H} 8 \mathrm{~B}$ & C8 & $\mathrm{H} 8 \mathrm{C}$ & 109.5 \\
\hline $\mathrm{C} 2$ & C12 & $\mathrm{H} 12$ & 121.0 & C11 & C12 & $\mathrm{H} 12$ & 121.0 \\
\hline C16 & C17 & $\mathrm{H} 17$ & 119.7 & C18 & C17 & $\mathrm{H} 17$ & 119.7 \\
\hline C18 & N19 & H19 & 119.8 & C20 & N19 & H19 & 119.8 \\
\hline $\mathrm{C} 13$ & $\mathrm{C} 21$ & $\mathrm{H} 21$ & 127.3 & C20 & $\mathrm{C} 21$ & $\mathrm{H} 21$ & 127.3 \\
\hline C16 & $\mathrm{N} 22$ & $\mathrm{H} 22$ & 117.5 & $\mathrm{C} 23$ & N22 & $\mathrm{H} 22$ & 117.5 \\
\hline N22 & $\mathrm{C} 23$ & $\mathrm{H} 23 \mathrm{~A}$ & 108.6 & N22 & $\mathrm{C} 23$ & $\mathrm{H} 23 \mathrm{~B}$ & 108.6 \\
\hline C24 & $\mathrm{C} 23$ & $\mathrm{H} 23 \mathrm{~A}$ & 108.6 & C24 & $\mathrm{C} 23$ & $\mathrm{H} 23 \mathrm{~B}$ & 108.6 \\
\hline $\mathrm{H} 23 \mathrm{~A}$ & $\mathrm{C} 23$ & $\mathrm{H} 23 \mathrm{~B}$ & 107.6 & C24 & $\mathrm{O} 25$ & $\mathrm{H} 25$ & 109.5 \\
\hline C24 & C26 & $\mathrm{H} 26 \mathrm{~A}$ & 109.5 & C24 & C26 & H26B & 109.5 \\
\hline C24 & C26 & $\mathrm{H} 26 \mathrm{C}$ & 109.5 & $\mathrm{H} 26 \mathrm{~A}$ & $\mathrm{C} 26$ & H26B & 109.5 \\
\hline $\mathrm{H} 26 \mathrm{~A}$ & C26 & $\mathrm{H} 26 \mathrm{C}$ & 109.5 & H26B & $\mathrm{C} 26$ & $\mathrm{H} 26 \mathrm{C}$ & 109.5 \\
\hline C24 & C27 & $\mathrm{H} 27 \mathrm{~A}$ & 109.5 & C24 & C27 & $\mathrm{H} 27 \mathrm{~B}$ & 109.5 \\
\hline C24 & $\mathrm{C} 27$ & $\mathrm{H} 27 \mathrm{C}$ & 109.5 & $\mathrm{H} 27 \mathrm{~A}$ & C27 & H27B & 109.5 \\
\hline $\mathrm{H} 27 \mathrm{~A}$ & $\mathrm{C} 27$ & $\mathrm{H} 27 \mathrm{C}$ & 109.5 & H27B & $\mathrm{C} 27$ & $\mathrm{H} 27 \mathrm{C}$ & 109.5 \\
\hline N28 & C29 & $\mathrm{H} 29 \mathrm{~A}$ & 110.9 & N28 & C29 & $\mathrm{H} 29 \mathrm{~B}$ & 110.9 \\
\hline C30 & C29 & $\mathrm{H} 29 \mathrm{~A}$ & 110.9 & C30 & C29 & $\mathrm{H} 29 \mathrm{~B}$ & 110.9 \\
\hline $\mathrm{H} 29 \mathrm{~A}$ & C29 & $\mathrm{H} 29 \mathrm{~B}$ & 108.9 & C29 & C30 & $\mathrm{H} 30$ & 112.1 \\
\hline C31 & C30 & $\mathrm{H} 30$ & 112.1 & F33 & C30 & $\mathrm{H} 30$ & 112.1 \\
\hline C30 & C31 & $\mathrm{H} 31 \mathrm{~A}$ & 111.0 & C30 & C31 & H31B & 111.0 \\
\hline C32 & C31 & H31A & 111.0 & C32 & C31 & H31B & 111.0 \\
\hline H31A & C31 & H31B & 109.0 & N28 & C32 & $\mathrm{H} 32 \mathrm{~A}$ & 111.4 \\
\hline N28 & C32 & $\mathrm{H} 32 \mathrm{~B}$ & 111.4 & C31 & C32 & $\mathrm{H} 32 \mathrm{~A}$ & 111.4 \\
\hline C31 & C32 & H32B & 111.4 & $\mathrm{H} 32 \mathrm{~A}$ & C32 & H32B & 109.3 \\
\hline C35 & C36 & H36 & 120.8 & C37 & C36 & H36 & 120.8 \\
\hline C36 & C37 & H37 & 119.9 & C38 & C37 & H37 & 119.9 \\
\hline C35 & C44 & $\mathrm{H} 44$ & 121.5 & C43 & C44 & $\mathrm{H} 44$ & 121.5 \\
\hline C40 & C45 & $\mathrm{H} 45 \mathrm{~A}$ & 109.5 & C40 & C45 & $\mathrm{H} 45 \mathrm{~B}$ & 109.5 \\
\hline C40 & C45 & $\mathrm{H} 45 \mathrm{C}$ & 109.5 & $\mathrm{H} 45 \mathrm{~A}$ & C45 & $\mathrm{H} 45 \mathrm{~B}$ & 109.5 \\
\hline $\mathrm{H} 45 \mathrm{~A}$ & C45 & $\mathrm{H} 45 \mathrm{C}$ & 109.5 & $\mathrm{H} 45 \mathrm{~B}$ & C45 & $\mathrm{H} 45 \mathrm{C}$ & 109.5 \\
\hline
\end{tabular}




$\begin{array}{llllllll}\text { C49 } & \text { C50 } & \text { H50 } & 119.7 & \text { C51 } & \text { C50 } & \text { H50 } & 119.7 \\ \text { C51 } & \text { N52 } & \text { H52 } & 119.3 & \text { C53 } & \text { N52 } & \text { H52 } & 119.4 \\ \text { C46 } & \text { C54 } & \text { H54 } & 128.1 & \text { C53 } & \text { C54 } & \text { H54 } & 128.1 \\ \text { C49 } & \text { N55 } & \text { H55 } & 117.8 & \text { C56 } & \text { N55 } & \text { H55 } & 117.8 \\ \text { N55 } & \text { C56 } & \text { H56A } & 108.6 & \text { N55 } & \text { C56 } & \text { H56B } & 108.6 \\ \text { C57 } & \text { C56 } & \text { H56A } & 108.6 & \text { C57 } & \text { C56 } & \text { H56B } & 108.6 \\ \text { H56A } & \text { C56 } & \text { H56B } & 107.6 & \text { C57 } & \text { O58 } & \text { H58 } & 109.5 \\ \text { C57 } & \text { C59 } & \text { H59A } & 109.5 & \text { C57 } & \text { C59 } & \text { H59B } & 109.5 \\ \text { C57 } & \text { C59 } & \text { H59C } & 109.5 & \text { H59A } & \text { C59 } & \text { H59B } & 109.5 \\ \text { H59A } & \text { C59 } & \text { H59C } & 109.5 & \text { H59B } & \text { C59 } & \text { H59C } & 109.5 \\ \text { C57 } & \text { C60 } & \text { H60A } & 109.5 & \text { C57 } & \text { C60 } & \text { H60B } & 109.5 \\ \text { C57 } & \text { C60 } & \text { H60C } & 109.5 & \text { H60A } & \text { C60 } & \text { H60B } & 109.5 \\ \text { H60A } & \text { C60 } & \text { H60C } & 109.5 & \text { H60B } & \text { C60 } & \text { H60C } & 109.5 \\ \text { N61 } & \text { C62 } & \text { H62A } & 111.1 & \text { N61 } & \text { C62 } & \text { H62B } & 111.1 \\ \text { C63 } & \text { C62 } & \text { H62A } & 111.1 & \text { C63 } & \text { C62 } & \text { H62B } & 111.1 \\ \text { H62A } & \text { C62 } & \text { H62B } & 109.1 & \text { C62 } & \text { C63 } & \text { H63 } & 111.6 \\ \text { C64 } & \text { C63 } & \text { H63 } & 111.6 & \text { F66 } & \text { C63 } & \text { H63 } & 111.6 \\ \text { C63 } & \text { C64 } & \text { H64A } & 111.0 & \text { C63 } & \text { C64 } & \text { H64B } & 111.0 \\ \text { C65 } & \text { C64 } & \text { H64A } & 111.0 & \text { C65 } & \text { C64 } & \text { H64B } & 111.0 \\ \text { H64A } & \text { C64 } & \text { H64B } & 109.0 & \text { N61 } & \text { C65 } & \text { H65A } & 111.5 \\ \text { N61 } & \text { C65 } & \text { H65B } & 111.5 & \text { C64 } & \text { C65 } & \text { H65A } & 111.5 \\ \text { C64 } & \text { C65 } & \text { H65B } & 111.5 & \text { H65A } & \text { C65 } & \text { H65B } & 109.4\end{array}$


Table S9. Torsion Angles( $\left({ }^{\circ}\right)$

(Those having bond angles $>160$ or $<20$ degrees are excluded.)

atom1 atom2 atom3 atom4 angle

$\begin{array}{lllll}\mathrm{F} 1 & \mathrm{C} 2 & \mathrm{C} 3 & \mathrm{C} 4 & 179.5(5)\end{array}$

C3 $\quad$ C2 $\quad$ C12 $\mathrm{C} 11 \quad 0.0(8)$

$\mathrm{C} 2 \quad \mathrm{C} 3 \quad \mathrm{C} 4 \quad \mathrm{C} 5 \quad-0.4(8)$

$\mathrm{C} 3 \quad \mathrm{C} 4 \quad \mathrm{C} 5 \quad \mathrm{C} 11 \quad 1.5(8)$

C4 $\quad$ C5 $\quad$ C11 N10 $178.7(4)$

N6 $\quad$ C5 $\quad$ C11 N10 $\quad-0.0(7)$

$\begin{array}{lllll}\mathrm{C} 11 & \mathrm{C} 5 & \mathrm{~N} 6 & \mathrm{C} 7 & -3.1(7)\end{array}$

$\begin{array}{lllll}\mathrm{C} 5 & \mathrm{~N} 6 & \mathrm{C} 7 & \mathrm{C} 9 & 4.3(7)\end{array}$

N6 $\quad \mathrm{C} 7 \quad \mathrm{C} 9 \quad \mathrm{C} 13 \quad 177.0(4)$

$\begin{array}{lllll}\mathrm{C} 8 & \mathrm{C} 7 & \mathrm{C} 9 & \mathrm{C} 13 & 0.7(7)\end{array}$

$\begin{array}{lllll}\mathrm{C} 7 & \mathrm{C} 9 & \mathrm{C} 13 & \mathrm{~N} 14 & 16.4(7)\end{array}$

N10 $\quad$ C9 $\quad$ C13 $1314 \quad-164.0(4)$

C13 C9 N10 C11 $179.8(3)$

C9 $\quad \mathrm{N} 10 \quad \mathrm{C} 11 \quad \mathrm{C} 12 \quad-177.6(4)$

N10 C11 C12 C2 $-179.5(4)$

C9 $\quad \mathrm{C} 13 \quad \mathrm{C} 21 \quad \mathrm{C} 20 \quad-178.3(4)$

$\mathrm{C} 21 \quad \mathrm{C} 13 \quad \mathrm{~N} 14 \quad \mathrm{~N} 15 \quad 0.8(4)$

C13 N14 N15 C20 $-0.1(4)$

N14 N15 C16 N22 5.1(5)

N14 N15 C20 C21 $-0.7(4)$

C16 N15 C20 C21 179.6(3)

$\begin{array}{lllll}\mathrm{C} 20 & \mathrm{~N} 15 & \mathrm{C} 16 & \mathrm{~N} 22 & -175.2(3)\end{array}$

$\mathrm{N} 15 \quad \mathrm{C} 16 \quad \mathrm{~N} 22 \quad \mathrm{C} 23 \quad 174.6(3)$

N22 $\mathrm{C} 16 \quad \mathrm{C} 17 \quad \mathrm{C} 18 \quad 175.4(4)$

$\mathrm{C} 16 \quad \mathrm{C} 17 \quad \mathrm{C} 18 \quad \mathrm{~N} 28 \quad 179.7(3)$

$\begin{array}{lllll}\mathrm{C} 17 & \mathrm{C} 18 & \mathrm{~N} 28 & \mathrm{C} 29 & -7.8(6)\end{array}$

$\mathrm{N} 19 \quad \mathrm{C} 18 \quad \mathrm{~N} 28 \quad \mathrm{C} 29 \quad 171.7(3)$

N28 $\mathrm{C} 18 \quad \mathrm{~N} 19 \quad \mathrm{C} 20 \quad-177.5(3)$

C18 N19 C20 C21 $177.6(4)$

N19 C20 C21 C13 $\quad-178.3(4)$

$\mathrm{N} 22 \quad \mathrm{C} 23 \quad \mathrm{C} 24 \quad \mathrm{O} 25 \quad-73.0(4)$

$\mathrm{N} 22 \quad \mathrm{C} 23 \quad \mathrm{C} 24 \quad \mathrm{C} 27 \quad 46.2(5)$

C18 N28 C32 C31 $-174.1(3)$

$\begin{array}{lllll}\text { C32 } & \text { N28 } & \text { C29 } & \text { C30 } & 2.9(4)\end{array}$ atom1 atom2 atom3 atom4 angle

F1 C2 $\quad$ C12 C11 $-179.9(4)$

$\mathrm{C} 12 \quad \mathrm{C} 2 \quad \mathrm{C} 3 \quad \mathrm{C} 4 \quad-0.4(9)$

C3 $\mathrm{C} 4 \quad \mathrm{C} 5 \quad \mathrm{~N} 6 \quad-179.7(5)$

$\begin{array}{lllll}\mathrm{C} 4 & \mathrm{C} 5 & \mathrm{~N} 6 & \mathrm{C} 7 & 178.2(4)\end{array}$

C4 $\quad$ C5 $\quad$ C11 $11212 \quad-1.9(7)$

N6 $\quad$ C5 $\quad$ C11 C12 179.3(4)

C5 $\quad \mathrm{N} 6 \quad \mathrm{C} 7 \quad \mathrm{C} 8 \quad-179.3(4)$

N6 $\quad \mathrm{C} 7 \quad \mathrm{C} 9 \quad \mathrm{~N} 10 \quad-2.6(7)$

$\mathrm{C} 8 \quad \mathrm{C} 7 \quad \mathrm{C} 9 \quad \mathrm{~N} 10 \quad-178.9(4)$

$\mathrm{C} 7 \quad \mathrm{C} 9 \quad \mathrm{~N} 10 \quad \mathrm{C} 11 \quad-0.5(6)$

$\mathrm{C} 7 \quad \mathrm{C} 9 \quad \mathrm{C} 13 \quad \mathrm{C} 21 \quad-166.8(4)$

$\mathrm{N} 10 \quad \mathrm{C} 9 \quad \mathrm{C} 13 \quad \mathrm{C} 21 \quad 12.9(6)$

C9 $\mathrm{N} 10 \quad \mathrm{C} 11 \quad \mathrm{C} 5 \quad 1.8(6)$

C5 $\quad \mathrm{C} 11 \quad \mathrm{C} 12 \quad \mathrm{C} 2 \quad 1.2(7)$

$\begin{array}{lllll}\mathrm{C} 9 & \mathrm{C} 13 & \mathrm{~N} 14 & \mathrm{~N} 15 & 178.1(3)\end{array}$

$\mathrm{N} 14 \quad \mathrm{C} 13 \quad \mathrm{C} 21 \quad \mathrm{C} 20 \quad-1.2(5)$

C13 N14 N15 C16 179.7(3)

N14 N15 C16 $\quad$ C17 $-176.7(3)$

N14 N15 C20 N19 178.8(3)

C16 N15 C20 N19 $-0.9(6)$

C20 N15 C16 C17 3.0(5)

$\begin{array}{lllll}\mathrm{N} 15 & \mathrm{C} 16 & \mathrm{C} 17 & \mathrm{C} 18 & -2.6(6)\end{array}$

$\mathrm{C} 17 \quad \mathrm{C} 16 \quad \mathrm{~N} 22 \quad \mathrm{C} 23 \quad-3.3(6)$

$\begin{array}{lllll}\mathrm{C} 16 & \mathrm{C} 17 & \mathrm{C} 18 & \mathrm{~N} 19 & 0.2(6)\end{array}$

$\begin{array}{lllll}\mathrm{C} 17 & \mathrm{C} 18 & \mathrm{~N} 19 & \mathrm{C} 20 & 2.0(6)\end{array}$

$\begin{array}{lllll}\mathrm{C} 17 & \mathrm{C} 18 & \mathrm{~N} 28 & \mathrm{C} 32 & -173.7(3)\end{array}$

N19 $\mathrm{C} 18 \quad \mathrm{~N} 28 \quad \mathrm{C} 32 \quad 5.8(6)$

C18 N19 C20 N15 $-1.7(6)$

$\mathrm{N} 15 \quad \mathrm{C} 20 \quad \mathrm{C} 21 \quad \mathrm{C} 13 \quad 1.1(4)$

C16 N22 $\mathrm{C}_{23} \mathrm{C} 24 \quad 88.8(4)$

$\mathrm{N} 22 \quad \mathrm{C} 23 \quad \mathrm{C} 24 \quad \mathrm{C} 26 \quad 169.0(3)$

C18 N28 C29 C30 $-164.5(3)$

C29 N28 C32 C31 18.6(4)

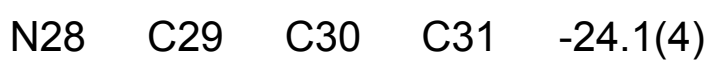




\begin{tabular}{|c|c|c|c|c|c|c|c|c|c|}
\hline N28 & $\mathrm{C} 29$ & C30 & F33 & $90.2(4)$ & C29 & C30 & C31 & C32 & $35.7(5)$ \\
\hline F33 & C30 & C31 & C32 & $-78.6(5)$ & C30 & C31 & C32 & N28 & $-32.9(5)$ \\
\hline F34 & C35 & C36 & C37 & $-179.3(4)$ & F34 & C35 & C44 & $\mathrm{C} 43$ & $-179.0(4)$ \\
\hline C36 & C35 & C44 & C43 & $-1.4(8)$ & C44 & C35 & C36 & C37 & $3.1(9)$ \\
\hline C35 & C36 & C37 & C38 & $-1.9(8)$ & C36 & C37 & $\mathrm{C} 38$ & N39 & $178.4(5)$ \\
\hline C36 & C37 & C38 & $\mathrm{C} 43$ & $-0.6(8)$ & C37 & C38 & N39 & $\mathrm{C} 40$ & $177.7(4)$ \\
\hline C37 & C38 & $\mathrm{C} 43$ & $\mathrm{~N} 42$ & $-177.9(4)$ & C37 & C38 & $\mathrm{C} 43$ & C44 & $2.3(7)$ \\
\hline N39 & C38 & $\mathrm{C} 43$ & $\mathrm{~N} 42$ & $3.1(7)$ & N39 & C38 & $\mathrm{C} 43$ & C44 & $-176.7(4)$ \\
\hline C43 & C38 & N39 & C40 & $-3.3(7)$ & C38 & N39 & C40 & C41 & $1.8(6)$ \\
\hline C38 & N39 & $\mathrm{C} 40$ & $\mathrm{C} 45$ & $-179.7(4)$ & N39 & $\mathrm{C} 40$ & C41 & N42 & $0.0(7)$ \\
\hline N39 & C40 & C41 & C46 & $178.5(4)$ & C45 & C40 & C41 & N42 & $-178.4(4)$ \\
\hline C45 & C40 & C41 & C46 & $0.1(7)$ & C40 & C41 & N42 & C43 & $-0.3(6)$ \\
\hline $\mathrm{C} 40$ & C41 & $\mathrm{C} 46$ & N47 & $-11.7(7)$ & $\mathrm{C} 40$ & C41 & $\mathrm{C} 46$ & C54 & $169.9(4)$ \\
\hline N42 & C41 & $\mathrm{C} 46$ & N47 & $166.9(4)$ & N42 & C41 & C46 & C54 & $-11.5(6)$ \\
\hline C46 & C41 & N42 & C43 & $-178.9(3)$ & C41 & N42 & C43 & C38 & $-1.2(6)$ \\
\hline C41 & N42 & C43 & C44 & $178.6(4)$ & C38 & C43 & C44 & C35 & $-1.3(7)$ \\
\hline N42 & $\mathrm{C} 43$ & C44 & C35 & $178.8(4)$ & C41 & C46 & N47 & N48 & $-177.5(3)$ \\
\hline C41 & C46 & C54 & C53 & $177.4(3)$ & N47 & C46 & C54 & C53 & $-1.2(4)$ \\
\hline C54 & C46 & N47 & N48 & $1.1(4)$ & C46 & N47 & N48 & C49 & $179.5(3)$ \\
\hline C46 & N47 & N48 & C53 & $-0.6(4)$ & N47 & N48 & C49 & C50 & $177.0(3)$ \\
\hline N47 & N48 & C49 & N55 & $-2.6(5)$ & N47 & N48 & C53 & N52 & $179.0(3)$ \\
\hline N47 & N48 & C53 & C54 & $-0.1(4)$ & C49 & N48 & C53 & N52 & $-1.1(6)$ \\
\hline C49 & N48 & C53 & C54 & 179.8(3) & C53 & N48 & C49 & C50 & $-2.9(6)$ \\
\hline C53 & N48 & C49 & N55 & $177.5(3)$ & N48 & C49 & C50 & C51 & $4.3(6)$ \\
\hline N48 & C49 & N55 & C56 & $-175.6(3)$ & C50 & C49 & N55 & C56 & $4.8(6)$ \\
\hline N55 & C49 & C50 & C51 & $-176.1(4)$ & C49 & C50 & C51 & N52 & $-2.0(6)$ \\
\hline C49 & C50 & C51 & N61 & $176.9(4)$ & C50 & C51 & N52 & C53 & $-2.2(6)$ \\
\hline C50 & C51 & N61 & C62 & $167.9(3)$ & C50 & C51 & N61 & C65 & $2.9(6)$ \\
\hline N52 & C51 & N61 & C62 & $-13.2(5)$ & N52 & C51 & N61 & C65 & $-178.2(3)$ \\
\hline N61 & C51 & N52 & C53 & 178.9(3) & C51 & N52 & C53 & N48 & $3.7(6)$ \\
\hline C51 & N52 & C53 & C54 & $-177.6(4)$ & N48 & C53 & C54 & C46 & $0.7(4)$ \\
\hline N52 & $\mathrm{C} 53$ & C54 & C46 & $-178.2(4)$ & C49 & N55 & C56 & C57 & $-91.0(4)$ \\
\hline N55 & C56 & C57 & O58 & $74.2(4)$ & N55 & C56 & C57 & C59 & $-45.2(5)$ \\
\hline N55 & C56 & C57 & $\mathrm{C} 60$ & $-168.3(3)$ & C51 & N61 & C62 & C63 & $-159.5(3)$ \\
\hline C51 & N61 & C65 & C64 & $-176.8(3)$ & C62 & N61 & $\mathrm{C} 65$ & C64 & $16.7(4)$ \\
\hline C65 & N61 & $\mathrm{C} 62$ & $\mathrm{C} 63$ & $6.9(4)$ & N61 & C62 & $\mathrm{C} 63$ & C64 & $-28.3(4)$ \\
\hline N61 & C62 & $\mathrm{C} 63$ & F66 & $87.1(4)$ & $\mathrm{C} 62$ & C63 & C64 & C65 & $39.4(4)$ \\
\hline F66 & C63 & C64 & C65 & $-76.8(4)$ & C63 & C64 & C65 & N61 & $-33.7(4)$ \\
\hline
\end{tabular}


Table S10. Possible hydrogen bonds

$\begin{array}{llllllll}\text { Donor } & \mathrm{H} & \text { Acceptor } & \text { D...A } & \text { D-H } & \text { H...A } & \text { D-H...A } & \\ \text { N19 } & \mathrm{H} 19 & \text { Cl68 } & 3.094(3) & 0.86 & 2.37 & 142.02 & \\ \text { N22 } & \mathrm{H} 22 & \mathrm{~N} 14 & 2.734(5) & 0.86 & 2.35 & 107.48 & \text { intramol. } \\ \text { O25 } & \mathrm{H} 25 & \mathrm{Cl} 67^{1} & 3.182(3) & 0.82 & 2.38 & 166.23 & \\ \text { N52 } & \mathrm{H} 52 & \mathrm{Cl} 67 & 3.085(3) & 0.86 & 2.35 & 143.45 & \\ \text { N55 } & \mathrm{H} 55 & \mathrm{~N} 47 & 2.732(5) & 0.86 & 2.35 & 107.63 & \text { intramol. } \\ \text { O58 } & \mathrm{H} 58 & \mathrm{Cl}^{2} & 3.202(3) & 0.82 & 2.40 & 165.72 & \end{array}$

Symmetry Operators:

(1) $-X+1, Y+1 / 2,-Z$

(2) $-\mathrm{X}+2, \mathrm{Y}+1 / 2-1,-\mathrm{Z}+1$ 
Table S11. Intramolecular contacts less than $3.60 \AA$

\begin{tabular}{|c|c|c|c|c|c|}
\hline atom & atom & distance & atom & atom & distance \\
\hline F1 & $\mathrm{C} 4$ & $3.585(6)$ & $\mathrm{C} 2$ & C5 & $2.747(7)$ \\
\hline C3 & C11 & $2.772(7)$ & C4 & C12 & $2.791(7)$ \\
\hline C5 & C9 & $2.698(6)$ & N6 & N10 & $2.812(6)$ \\
\hline $\mathrm{C} 7$ & C11 & $2.719(6)$ & $\mathrm{C} 7$ & N14 & $3.037(6)$ \\
\hline $\mathrm{C} 8$ & C13 & $3.083(7)$ & $\mathrm{C} 8$ & N14 & $2.872(6)$ \\
\hline $\mathrm{Cg}$ & $\mathrm{C} 12$ & $3.584(6)$ & C9 & N15 & $3.546(6)$ \\
\hline N10 & N14 & $3.582(5)$ & N10 & C21 & $2.823(6)$ \\
\hline $\mathrm{C} 13$ & C16 & $3.470(6)$ & C13 & N19 & $3.550(6)$ \\
\hline N14 & N19 & $3.535(5)$ & N14 & N22 & $2.734(5)$ \\
\hline N15 & C18 & $2.709(6)$ & C16 & N19 & $2.790(6)$ \\
\hline C16 & $\mathrm{C} 21$ & $3.517(6)$ & C16 & C24 & $3.341(6)$ \\
\hline C16 & $\mathrm{O} 25$ & $3.270(5)$ & C17 & C20 & $2.754(6)$ \\
\hline C17 & $\mathrm{C} 23$ & $3.005(7)$ & C17 & $\mathrm{O} 25$ & $3.075(5)$ \\
\hline C17 & C29 & $2.885(7)$ & N19 & C32 & $2.796(6)$ \\
\hline $\mathrm{C} 20$ & N22 & $3.581(6)$ & $\mathrm{C} 20$ & N28 & $3.570(6)$ \\
\hline N22 & $\mathrm{O} 25$ & $3.002(4)$ & N22 & $\mathrm{C} 27$ & 2.868(5) \\
\hline N28 & F33 & $3.019(5)$ & $\mathrm{C} 32$ & F33 & 2.915(5) \\
\hline F34 & C37 & $3.582(6)$ & C35 & C38 & $2.736(7)$ \\
\hline C35 & N42 & $3.596(6)$ & C36 & $\mathrm{C} 43$ & $2.790(7)$ \\
\hline C37 & C44 & $2.813(8)$ & C38 & C41 & $2.687(6)$ \\
\hline N39 & N42 & $2.804(6)$ & $\mathrm{C} 40$ & $\mathrm{C} 43$ & $2.723(7)$ \\
\hline C40 & N47 & $3.029(6)$ & C41 & C44 & $3.584(6)$ \\
\hline C41 & N48 & $3.559(5)$ & N42 & N47 & $3.585(5)$ \\
\hline N42 & C54 & $2.806(6)$ & $\mathrm{C} 45$ & C46 & $3.099(7)$ \\
\hline C45 & N47 & $2.874(6)$ & C46 & C49 & $3.469(6)$ \\
\hline C46 & N52 & $3.547(6)$ & N47 & N52 & $3.533(5)$ \\
\hline N47 & N55 & $2.732(5)$ & N48 & C51 & $2.711(6)$ \\
\hline C49 & N52 & $2.784(6)$ & C49 & C54 & $3.544(6)$ \\
\hline C49 & C57 & $3.361(6)$ & C49 & O58 & $3.293(5)$ \\
\hline C50 & C53 & $2.762(6)$ & C50 & C56 & $3.000(7)$ \\
\hline C50 & O58 & $3.104(5)$ & C50 & C65 & $2.885(7)$ \\
\hline N52 & C62 & $2.807(6)$ & C53 & N55 & $3.585(6)$ \\
\hline C53 & N61 & $3.574(6)$ & N55 & O58 & $3.012(4)$ \\
\hline N55 & C59 & $2.901(5)$ & N61 & F66 & $2.980(5)$ \\
\hline C65 & F66 & $2.889(6)$ & & & \\
\hline
\end{tabular}


Table S12. Intramolecular contacts less than $3.60 \AA$ involving hydrogens

$\begin{array}{llllll}\text { atom } & \text { atom } & \text { distance } & \text { atom } & \text { atom } & \text { distance } \\ \text { F1 } & \mathrm{H} 3 & 2.517 & \mathrm{~F} 1 & \mathrm{H} 12 & 2.527 \\ \mathrm{C} 2 & \mathrm{H} 4 & 3.208 & \mathrm{C} 3 & \mathrm{H} 12 & 3.248 \\ \mathrm{C} 5 & \mathrm{H} 3 & 3.249 & \mathrm{C} 5 & \mathrm{H} 12 & 3.272 \\ \mathrm{~N} 6 & \mathrm{H} 4 & 2.590 & \mathrm{~N} 6 & \mathrm{H} 8 \mathrm{~A} & 3.024 \\ \mathrm{~N} 6 & \mathrm{H} 8 \mathrm{~B} & 3.003 & \mathrm{~N} 6 & \mathrm{H} 8 \mathrm{C} & 2.429 \\ \mathrm{C} 9 & \mathrm{H} 8 \mathrm{~A} & 2.764 & \mathrm{C} 9 & \mathrm{H} 8 \mathrm{~B} & 2.830 \\ \mathrm{C} 9 & \mathrm{H} 8 \mathrm{C} & 3.335 & \mathrm{C} 9 & \mathrm{H} 21 & 2.865 \\ \mathrm{~N} 10 & \mathrm{H} 12 & 2.562 & \mathrm{~N} 10 & \mathrm{H} 21 & 2.688 \\ \mathrm{C} 11 & \mathrm{H} 4 & 3.265 & \mathrm{C} 12 & \mathrm{H} 3 & 3.240 \\ \mathrm{C} 13 & \mathrm{H} 8 \mathrm{~A} & 2.971 & \mathrm{C} 13 & \mathrm{H} 8 \mathrm{~B} & 3.091 \\ \mathrm{~N} 14 & \mathrm{H} 8 \mathrm{~A} & 2.644 & \mathrm{~N} 14 & \mathrm{H} 8 \mathrm{~B} & 2.595 \\ \mathrm{~N} 14 & \mathrm{H} 21 & 3.174 & \mathrm{~N} 14 & \mathrm{H} 22 & 2.350 \\ \mathrm{~N} 15 & \mathrm{H} 17 & 3.194 & \mathrm{~N} 15 & \mathrm{H} 19 & 3.131 \\ \mathrm{~N} 15 & \mathrm{H} 21 & 3.096 & \mathrm{~N} 15 & \mathrm{H} 22 & 2.377 \\ \mathrm{C} 16 & \mathrm{H} 23 \mathrm{~A} & 2.592 & \mathrm{C} 16 & \mathrm{H} 23 \mathrm{~B} & 3.180 \\ \mathrm{C} 16 & \mathrm{H} 27 \mathrm{C} & 3.238 & \mathrm{C} 17 & \mathrm{H} 19 & 3.167 \\ \mathrm{C} 17 & \mathrm{H} 22 & 3.167 & \mathrm{C} 17 & \mathrm{H} 23 \mathrm{~A} & 2.727 \\ \mathrm{C} 17 & \mathrm{H} 29 \mathrm{~A} & 3.007 & \mathrm{C} 17 & \mathrm{H} 29 \mathrm{~B} & 2.742 \\ \mathrm{C} 18 & \mathrm{H} 29 \mathrm{~A} & 2.835 & \mathrm{C} 18 & \mathrm{H} 29 \mathrm{~B} & 2.662 \\ \mathrm{C} 18 & \mathrm{H} 32 \mathrm{~A} & 2.805 & \mathrm{C} 18 & \mathrm{H} 32 \mathrm{~B} & 2.740 \\ \mathrm{~N} 19 & \mathrm{H} 17 & 3.224 & \mathrm{~N} 19 & \mathrm{H} 21 & 2.874 \\ \mathrm{~N} 19 & \mathrm{H} 32 \mathrm{~A} & 2.908 & \mathrm{~N} 19 & \mathrm{H} 32 \mathrm{~B} & 2.706 \\ \mathrm{C} 21 & \mathrm{H} 19 & 2.741 & \mathrm{~N} 22 & \mathrm{H} 17 & 2.663 \\ \mathrm{~N} 22 & \mathrm{H} 27 \mathrm{~B} & 3.109 & \mathrm{~N} 22 & \mathrm{H} 27 \mathrm{C} & 2.511 \\ \mathrm{C} 23 & \mathrm{H} 17 & 2.757 & \mathrm{C} 23 & \mathrm{H} 25 & 3.094 \\ \mathrm{C} 23 & \mathrm{H} 26 \mathrm{~A} & 2.621 & \mathrm{C} 23 & \mathrm{H} 26 \mathrm{~B} & 2.690 \\ \mathrm{C} 23 & \mathrm{H} 26 \mathrm{C} & 3.311 & \mathrm{C} 23 & \mathrm{H} 27 \mathrm{~A} & 3.315 \\ \mathrm{C} 23 & \mathrm{H} 27 \mathrm{~B} & 2.689 & \mathrm{C} 23 & \mathrm{H} 27 \mathrm{C} & 2.646 \\ \mathrm{C} 24 & \mathrm{H} 17 & 3.230 & \mathrm{O} 24 & \mathrm{H} 22 & 2.956 \\ \mathrm{O} 25 & \mathrm{H} 17 & 2.505 & \mathrm{H} 23 \mathrm{~A} & 2.467 \\ \mathrm{O} 25 & \mathrm{H} 23 \mathrm{~B} & 3.196 & \mathrm{O} 25 & \mathrm{H} 26 \mathrm{~A} & 2.620 \\ \mathrm{O} 25 & \mathrm{H} 26 \mathrm{~B} & 3.244 & \mathrm{H} 29 \mathrm{~B} & 3.570 \\ \mathrm{O} 25 & \mathrm{H} 27 \mathrm{~A} & 2.593 & \mathrm{H} 25 & & 2.614 \\ \mathrm{O} 25 & \mathrm{H} 27 \mathrm{C} & 2.593 & & \mathrm{H} 27 \mathrm{~B} & 3.224 \\ & & & & & \end{array}$




\begin{tabular}{|c|c|c|c|c|c|}
\hline C26 & $\mathrm{H} 23 \mathrm{~A}$ & 2.719 & C26 & $\mathrm{H} 23 \mathrm{~B}$ & 2.557 \\
\hline C26 & $\mathrm{H} 25$ & 2.525 & C26 & $\mathrm{H} 27 \mathrm{~A}$ & 2.695 \\
\hline C26 & $\mathrm{H} 27 \mathrm{~B}$ & 2.670 & C26 & $\mathrm{H} 27 \mathrm{C}$ & 3.323 \\
\hline C27 & $\mathrm{H} 22$ & 2.938 & $\mathrm{C} 27$ & $\mathrm{H} 23 \mathrm{~A}$ & 3.303 \\
\hline $\mathrm{C} 27$ & $\mathrm{H} 23 \mathrm{~B}$ & 2.784 & $\mathrm{C} 27$ & $\mathrm{H} 25$ & 2.574 \\
\hline $\mathrm{C} 27$ & $\mathrm{H} 26 \mathrm{~A}$ & 3.318 & $\mathrm{C} 27$ & $\mathrm{H} 26 \mathrm{~B}$ & 2.673 \\
\hline $\mathrm{C} 27$ & $\mathrm{H} 26 \mathrm{C}$ & 2.705 & N28 & $\mathrm{H} 17$ & 2.597 \\
\hline N28 & H19 & 2.451 & N28 & $\mathrm{H} 30$ & 3.156 \\
\hline N28 & $\mathrm{H} 31 \mathrm{~A}$ & 2.737 & N28 & H31B & 3.155 \\
\hline C29 & $\mathrm{H} 17$ & 2.570 & C29 & $\mathrm{H} 31 \mathrm{~A}$ & 2.758 \\
\hline C29 & H31B & 3.207 & C29 & $\mathrm{H} 32 \mathrm{~A}$ & 2.945 \\
\hline C29 & H32B & 3.169 & C30 & $\mathrm{H} 32 \mathrm{~A}$ & 2.791 \\
\hline C30 & $\mathrm{H} 32 \mathrm{~B}$ & 3.206 & C31 & $\mathrm{H} 29 \mathrm{~A}$ & 2.853 \\
\hline C31 & $\mathrm{H} 29 \mathrm{~B}$ & 3.163 & C32 & $\mathrm{H} 19$ & 2.445 \\
\hline C32 & $\mathrm{H} 29 \mathrm{~A}$ & 3.051 & C32 & $\mathrm{H} 29 \mathrm{~B}$ & 3.086 \\
\hline C32 & $\mathrm{H} 30$ & 3.245 & F33 & $\mathrm{H} 29 \mathrm{~A}$ & 3.157 \\
\hline F33 & $\mathrm{H} 29 \mathrm{~B}$ & 2.387 & F33 & $\mathrm{H} 31 \mathrm{~A}$ & 3.181 \\
\hline F33 & H31B & 2.436 & F33 & $\mathrm{H} 32 \mathrm{~A}$ & 2.835 \\
\hline F34 & H36 & 2.502 & F34 & $\mathrm{H} 44$ & 2.518 \\
\hline C35 & $\mathrm{H} 37$ & 3.208 & C36 & $\mathrm{H} 44$ & 3.262 \\
\hline C38 & $\mathrm{H} 36$ & 3.259 & C38 & $\mathrm{H} 44$ & 3.295 \\
\hline N39 & $\mathrm{H} 37$ & 2.587 & N39 & $\mathrm{H} 45 \mathrm{~A}$ & 3.004 \\
\hline N39 & $\mathrm{H} 45 \mathrm{~B}$ & 2.420 & N39 & $\mathrm{H} 45 \mathrm{C}$ & 3.027 \\
\hline C41 & $\mathrm{H} 45 \mathrm{~A}$ & 2.858 & C41 & $\mathrm{H} 45 \mathrm{~B}$ & 3.359 \\
\hline C41 & $\mathrm{H} 45 \mathrm{C}$ & 2.815 & C41 & H54 & 2.852 \\
\hline N42 & $\mathrm{H} 44$ & 2.601 & N42 & H54 & 2.670 \\
\hline $\mathrm{C} 43$ & $\mathrm{H} 37$ & 3.279 & C44 & $\mathrm{H} 36$ & 3.252 \\
\hline C46 & $\mathrm{H} 45 \mathrm{~A}$ & 3.094 & C46 & $\mathrm{H} 45 \mathrm{C}$ & 3.011 \\
\hline N47 & $\mathrm{H} 45 \mathrm{~A}$ & 2.749 & N47 & $\mathrm{H} 45 \mathrm{C}$ & 2.508 \\
\hline N47 & H54 & 3.190 & N47 & H55 & 2.346 \\
\hline N48 & $\mathrm{H} 50$ & 3.200 & N48 & H52 & 3.119 \\
\hline N48 & H54 & 3.110 & N48 & H55 & 2.381 \\
\hline C49 & H56A & 3.171 & C49 & H56B & 2.578 \\
\hline C49 & H59B & 3.319 & C50 & H52 & 3.162 \\
\hline C50 & H55 & 3.165 & C50 & H56B & 2.709 \\
\hline C50 & $\mathrm{H} 65 \mathrm{~A}$ & 2.939 & C50 & H65B & 2.832 \\
\hline C51 & $\mathrm{H} 62 \mathrm{~A}$ & 2.887 & C51 & H62B & 2.663 \\
\hline C51 & $\mathrm{H} 65 \mathrm{~A}$ & 2.784 & C51 & H65B & 2.748 \\
\hline
\end{tabular}




\begin{tabular}{|c|c|c|c|c|c|}
\hline N52 & $\mathrm{H} 50$ & 3.221 & N52 & H54 & 2.900 \\
\hline N52 & $\mathrm{H} 62 \mathrm{~A}$ & 2.971 & N52 & H62B & 2.663 \\
\hline C54 & H52 & 2.748 & N55 & $\mathrm{H} 50$ & 2.664 \\
\hline N55 & H59B & 2.600 & N55 & H59C & 3.076 \\
\hline C56 & $\mathrm{H} 50$ & 2.753 & C56 & H58 & 3.086 \\
\hline C56 & H59A & 3.347 & C56 & H59B & 2.722 \\
\hline C56 & H59C & 2.694 & C56 & $\mathrm{H} 60 \mathrm{~A}$ & 2.592 \\
\hline C56 & $\mathrm{H} 60 \mathrm{~B}$ & 3.309 & C56 & $\mathrm{H} 60 \mathrm{C}$ & 2.714 \\
\hline C57 & H50 & 3.254 & C57 & H55 & 2.958 \\
\hline O58 & H50 & 2.529 & O58 & $\mathrm{H} 56 \mathrm{~A}$ & 3.191 \\
\hline O58 & H56B & 2.450 & O58 & H59A & 2.662 \\
\hline O58 & H59B & 2.587 & O58 & $\mathrm{H} 59 \mathrm{C}$ & 3.248 \\
\hline O58 & $\mathrm{H} 60 \mathrm{~A}$ & 2.637 & O58 & $\mathrm{H} 60 \mathrm{~B}$ & 2.596 \\
\hline O58 & $\mathrm{H} 60 \mathrm{C}$ & 3.244 & C59 & H55 & 2.952 \\
\hline C59 & H56A & 2.827 & C59 & H56B & 3.338 \\
\hline C59 & H58 & 2.481 & C59 & $\mathrm{H} 60 \mathrm{~A}$ & 3.334 \\
\hline C59 & H60B & 2.735 & C59 & $\mathrm{H} 60 \mathrm{C}$ & 2.662 \\
\hline C60 & H56A & 2.551 & $\mathrm{C} 60$ & H56B & 2.725 \\
\hline $\mathrm{C} 60$ & H58 & 2.653 & C60 & H59A & 2.675 \\
\hline C60 & H59B & 3.335 & C60 & $\mathrm{H} 59 \mathrm{C}$ & 2.723 \\
\hline N61 & H50 & 2.601 & N61 & H52 & 2.444 \\
\hline N61 & $\mathrm{H} 63$ & 3.153 & N61 & $\mathrm{H} 64 \mathrm{~A}$ & 2.715 \\
\hline N61 & H64B & 3.147 & C62 & H52 & 2.458 \\
\hline C62 & $\mathrm{H} 64 \mathrm{~A}$ & 2.717 & C62 & H64B & 3.208 \\
\hline C62 & $\mathrm{H} 65 \mathrm{~A}$ & 2.980 & C62 & H65B & 3.181 \\
\hline C63 & $\mathrm{H} 65 \mathrm{~A}$ & 2.779 & C63 & H65B & 3.206 \\
\hline C64 & $\mathrm{H} 62 \mathrm{~A}$ & 2.809 & C64 & H62B & 3.173 \\
\hline C65 & $\mathrm{H} 50$ & 2.563 & C65 & $\mathrm{H} 62 \mathrm{~A}$ & 3.046 \\
\hline C65 & H62B & 3.129 & C65 & $\mathrm{H} 63$ & 3.243 \\
\hline F66 & $\mathrm{H} 62 \mathrm{~A}$ & 3.169 & F66 & H62B & 2.423 \\
\hline F66 & $\mathrm{H} 64 \mathrm{~A}$ & 3.179 & F66 & H64B & 2.452 \\
\hline F66 & $\mathrm{H} 65 \mathrm{~A}$ & 2.803 & $\mathrm{H} 3$ & $\mathrm{H} 4$ & 2.287 \\
\hline $\mathrm{H} 8 \mathrm{~B}$ & $\mathrm{H} 22$ & 3.309 & $\mathrm{H} 17$ & $\mathrm{H} 22$ & 3.498 \\
\hline $\mathrm{H} 17$ & $\mathrm{H} 23 \mathrm{~A}$ & 2.294 & $\mathrm{H} 17$ & $\mathrm{H} 25$ & 3.202 \\
\hline $\mathrm{H} 17$ & $\mathrm{H} 29 \mathrm{~A}$ & 2.540 & $\mathrm{H} 17$ & $\mathrm{H} 29 \mathrm{~B}$ & 2.286 \\
\hline $\mathrm{H} 19$ & $\mathrm{H} 21$ & 2.834 & $\mathrm{H} 19$ & $\mathrm{H} 32 \mathrm{~A}$ & 2.484 \\
\hline $\mathrm{H} 19$ & H32B & 2.168 & $\mathrm{H} 22$ & $\mathrm{H} 23 \mathrm{~A}$ & 2.697 \\
\hline H22 & $\mathrm{H} 23 \mathrm{~B}$ & 2.211 & $\mathrm{H} 22$ & H27B & 2.971 \\
\hline
\end{tabular}




\begin{tabular}{|c|c|c|c|c|c|}
\hline $\mathrm{H} 22$ & $\mathrm{H} 27 \mathrm{C}$ & 2.499 & $\mathrm{H} 23 \mathrm{~A}$ & $\mathrm{H} 25$ & 3.219 \\
\hline $\mathrm{H} 23 \mathrm{~A}$ & $\mathrm{H} 26 \mathrm{~A}$ & 2.509 & $\mathrm{H} 23 \mathrm{~A}$ & $\mathrm{H} 26 \mathrm{~B}$ & 3.074 \\
\hline $\mathrm{H} 23 \mathrm{~A}$ & $\mathrm{H} 26 \mathrm{C}$ & 3.560 & $\mathrm{H} 23 \mathrm{~A}$ & $\mathrm{H} 27 \mathrm{~B}$ & 3.592 \\
\hline $\mathrm{H} 23 \mathrm{~A}$ & $\mathrm{H} 27 \mathrm{C}$ & 3.475 & $\mathrm{H} 23 \mathrm{~B}$ & $\mathrm{H} 26 \mathrm{~A}$ & 2.729 \\
\hline $\mathrm{H} 23 \mathrm{~B}$ & H26B & 2.413 & $\mathrm{H} 23 \mathrm{~B}$ & $\mathrm{H} 26 \mathrm{C}$ & 3.468 \\
\hline $\mathrm{H} 23 \mathrm{~B}$ & H27B & 2.644 & $\mathrm{H} 23 \mathrm{~B}$ & $\mathrm{H} 27 \mathrm{C}$ & 3.080 \\
\hline $\mathrm{H} 25$ & $\mathrm{H} 26 \mathrm{~A}$ & 2.798 & $\mathrm{H} 25$ & H26B & 3.399 \\
\hline $\mathrm{H} 25$ & $\mathrm{H} 26 \mathrm{C}$ & 2.402 & $\mathrm{H} 25$ & $\mathrm{H} 27 \mathrm{~A}$ & 2.455 \\
\hline $\mathrm{H} 25$ & H27B & 3.434 & $\mathrm{H} 25$ & $\mathrm{H} 27 \mathrm{C}$ & 2.872 \\
\hline $\mathrm{H} 26 \mathrm{~A}$ & $\mathrm{H} 27 \mathrm{~A}$ & 3.568 & $\mathrm{H} 26 \mathrm{~A}$ & $\mathrm{H} 27 \mathrm{~B}$ & 3.534 \\
\hline H26B & $\mathrm{H} 27 \mathrm{~A}$ & 2.962 & H26B & $\mathrm{H} 27 \mathrm{~B}$ & 2.488 \\
\hline $\mathrm{H} 26 \mathrm{~B}$ & $\mathrm{H} 27 \mathrm{C}$ & 3.543 & $\mathrm{H} 26 \mathrm{C}$ & $\mathrm{H} 27 \mathrm{~A}$ & 2.550 \\
\hline $\mathrm{H} 26 \mathrm{C}$ & H27B & 2.972 & $\mathrm{H} 26 \mathrm{C}$ & $\mathrm{H} 27 \mathrm{C}$ & 3.579 \\
\hline $\mathrm{H} 29 \mathrm{~A}$ & $\mathrm{H} 30$ & 2.259 & $\mathrm{H} 29 \mathrm{~A}$ & $\mathrm{H} 31 \mathrm{~A}$ & 2.920 \\
\hline $\mathrm{H} 29 \mathrm{~B}$ & $\mathrm{H} 30$ & 2.589 & $\mathrm{H} 29 \mathrm{~B}$ & $\mathrm{H} 32 \mathrm{~A}$ & 3.379 \\
\hline H3O & $\mathrm{H} 31 \mathrm{~A}$ & 2.288 & $\mathrm{H} 30$ & H31B & 2.515 \\
\hline $\mathrm{H} 31 \mathrm{~A}$ & $\mathrm{H} 32 \mathrm{~A}$ & 2.850 & $\mathrm{H} 31 \mathrm{~A}$ & H32B & 2.294 \\
\hline H31B & $\mathrm{H} 32 \mathrm{~A}$ & 2.297 & H31B & H32B & 2.572 \\
\hline H36 & $\mathrm{H} 37$ & 2.307 & $\mathrm{H} 45 \mathrm{C}$ & H55 & 3.240 \\
\hline $\mathrm{H} 50$ & H55 & 3.499 & $\mathrm{H} 50$ & H56B & 2.275 \\
\hline $\mathrm{H} 50$ & H58 & 3.205 & $\mathrm{H} 50$ & $\mathrm{H} 65 \mathrm{~A}$ & 2.481 \\
\hline H50 & H65B & 2.354 & H52 & H54 & 2.856 \\
\hline H52 & $\mathrm{H} 62 \mathrm{~A}$ & 2.465 & H52 & $\mathrm{H} 62 \mathrm{~B}$ & 2.208 \\
\hline H55 & $\mathrm{H} 56 \mathrm{~A}$ & 2.227 & H55 & H56B & 2.709 \\
\hline $\mathrm{H} 55$ & $\mathrm{H} 59 \mathrm{~B}$ & 2.584 & H55 & $\mathrm{H} 59 \mathrm{C}$ & 2.912 \\
\hline $\mathrm{H} 56 \mathrm{~A}$ & H59B & 3.177 & $\mathrm{H} 56 \mathrm{~A}$ & $\mathrm{H} 59 \mathrm{C}$ & 2.665 \\
\hline $\mathrm{H} 56 \mathrm{~A}$ & $\mathrm{H} 60 \mathrm{~A}$ & 2.677 & $\mathrm{H} 56 \mathrm{~A}$ & H60B & 3.472 \\
\hline $\mathrm{H} 56 \mathrm{~A}$ & $\mathrm{H} 60 \mathrm{C}$ & 2.441 & H56B & H58 & 3.249 \\
\hline H56B & H59B & 3.534 & H56B & $\mathrm{H} 60 \mathrm{~A}$ & 2.491 \\
\hline H56B & $\mathrm{H} 60 \mathrm{~B}$ & 3.548 & H56B & $\mathrm{H} 60 \mathrm{C}$ & 3.116 \\
\hline H58 & $\mathrm{H} 59 \mathrm{~A}$ & 2.413 & H58 & H59B & 2.663 \\
\hline H58 & $\mathrm{H} 59 \mathrm{C}$ & 3.382 & H58 & $\mathrm{H} 60 \mathrm{~A}$ & 3.001 \\
\hline H58 & H60B & 2.529 & H58 & $\mathrm{H} 60 \mathrm{C}$ & 3.487 \\
\hline H59A & $\mathrm{H} 60 \mathrm{~A}$ & 3.567 & $\mathrm{H} 59 \mathrm{~A}$ & H60B & 2.549 \\
\hline $\mathrm{H} 59 \mathrm{~A}$ & $\mathrm{H} 60 \mathrm{C}$ & 2.886 & $\mathrm{H} 59 \mathrm{~B}$ & H60B & 3.583 \\
\hline H59B & $\mathrm{H} 60 \mathrm{C}$ & 3.552 & $\mathrm{H} 59 \mathrm{C}$ & $\mathrm{H} 60 \mathrm{~A}$ & 3.567 \\
\hline $\mathrm{H} 59 \mathrm{C}$ & H60B & 3.066 & $\mathrm{H} 59 \mathrm{C}$ & $\mathrm{H} 60 \mathrm{C}$ & 2.522 \\
\hline $\mathrm{H} 62 \mathrm{~A}$ & $\mathrm{H} 63$ & 2.257 & $\mathrm{H} 62 \mathrm{~A}$ & H64A & 2.832 \\
\hline
\end{tabular}




$\begin{array}{llllll}\text { H62A } & \text { H65B } & 3.595 & \text { H62B } & \text { H63 } & 2.565 \\ \text { H62B } & \text { H65A } & 3.448 & \text { H63 } & \text { H64A } & 2.296 \\ \text { H63 } & \text { H64B } & 2.500 & \text { H64A } & \text { H65A } & 2.845 \\ \text { H64A } & \text { H65B } & 2.288 & \text { H64B } & \text { H65A } & 2.292 \\ \text { H64B } & \text { H65B } & 2.560 & & & \end{array}$


Table S13. Intermolecular contacts less than $3.60 \AA$

\begin{tabular}{|c|c|c|c|c|c|}
\hline atom & atom & distance & atom & atom & distance \\
\hline $\mathrm{F} 1$ & $\mathrm{C} 29^{1}$ & $2.988(5)$ & $\mathrm{F} 1$ & $\mathrm{C} 30^{1}$ & $3.418(6)$ \\
\hline $\mathrm{F} 1$ & $\mathrm{C} 35^{1}$ & $3.546(7)$ & F1 & $\mathrm{C} 36^{1}$ & $3.544(7)$ \\
\hline C3 & $\mathrm{O} 25^{1}$ & $3.473(6)$ & N6 & $\mathrm{C} 63^{2}$ & $3.588(6)$ \\
\hline C9 & C50 & $3.394(6)$ & $\mathrm{Cg}$ & C51 & $3.559(7)$ \\
\hline N10 & C50 & $3.388(6)$ & N10 & $\mathrm{F} 66^{3}$ & $3.489(5)$ \\
\hline C12 & $\mathrm{F} 66^{3}$ & $3.350(6)$ & $\mathrm{C} 13$ & $\mathrm{C} 49$ & $3.403(6)$ \\
\hline C13 & C50 & $3.401(6)$ & N14 & N48 & $3.571(5)$ \\
\hline N14 & N52 & $3.374(5)$ & N14 & C53 & $3.390(6)$ \\
\hline N15 & N47 & $3.562(4)$ & N15 & N48 & $3.337(5)$ \\
\hline N15 & C53 & $3.355(6)$ & N15 & C54 & $3.568(6)$ \\
\hline C16 & C46 & $3.387(5)$ & $\mathrm{C} 16$ & C53 & $3.599(6)$ \\
\hline C16 & C54 & $3.289(6)$ & $\mathrm{C} 17$ & $\mathrm{C} 27^{4}$ & $3.483(6)$ \\
\hline C17 & C41 & $3.339(6)$ & C17 & N42 & $3.394(6)$ \\
\hline C17 & C46 & $3.373(6)$ & $\mathrm{C} 18$ & $\mathrm{C} 27^{4}$ & $3.442(6)$ \\
\hline C18 & C41 & $3.537(7)$ & $\mathrm{C} 18$ & $\mathrm{C} 46$ & $3.596(7)$ \\
\hline N19 & N47 & $3.378(5)$ & N19 & Cl68 & $3.094(3)$ \\
\hline C20 & N47 & $3.362(5)$ & $\mathrm{C} 20$ & N48 & $3.343(6)$ \\
\hline $\mathrm{C} 20$ & C49 & $3.590(6)$ & $\mathrm{C} 21$ & N48 & $3.539(6)$ \\
\hline C21 & C49 & $3.261(6)$ & $\mathrm{C} 21$ & N55 & $3.317(5)$ \\
\hline N22 & C54 & $3.308(5)$ & N22 & $\mathrm{Cl} 67$ & $3.315(4)$ \\
\hline $\mathrm{O} 25$ & $C 3^{5}$ & $3.473(6)$ & $\mathrm{O} 25$ & $\mathrm{Cl} 67^{4}$ & $3.182(3)$ \\
\hline $\mathrm{C} 27$ & $\mathrm{C} 17^{6}$ & $3.483(6)$ & $\mathrm{C} 27$ & $\mathrm{C} 18^{6}$ & $3.442(6)$ \\
\hline N28 & $\mathrm{C} 40$ & $3.576(6)$ & $\mathrm{C} 29$ & $\mathrm{~F} 1^{5}$ & $2.988(5)$ \\
\hline C30 & $F 1^{5}$ & $3.418(6)$ & C31 & N397 & $3.390(6)$ \\
\hline F33 & $\mathrm{C} 44^{3}$ & $3.545(6)$ & F34 & $\mathrm{C} 64^{5}$ & $3.469(5)$ \\
\hline F34 & $C 65^{5}$ & $2.975(5)$ & $\mathrm{C} 35$ & $\mathrm{~F} 1^{5}$ & $3.546(7)$ \\
\hline C36 & $\mathrm{F} 1^{5}$ & $3.544(7)$ & $\mathrm{C} 36$ & $058^{5}$ & $3.488(6)$ \\
\hline N39 & $\mathrm{C} 31^{8}$ & $3.390(6)$ & C40 & N28 & $3.576(6)$ \\
\hline C41 & C17 & $3.339(6)$ & C41 & $\mathrm{C} 18$ & $3.537(7)$ \\
\hline N42 & C17 & $3.394(6)$ & $\mathrm{C} 44$ & F339 & $3.545(6)$ \\
\hline C46 & C16 & $3.387(5)$ & $\mathrm{C} 46$ & C17 & $3.373(6)$ \\
\hline C46 & C18 & $3.596(7)$ & $\mathrm{N} 47$ & N15 & $3.562(4)$ \\
\hline N47 & N19 & $3.378(5)$ & N47 & $\mathrm{C} 20$ & $3.362(5)$ \\
\hline N48 & N14 & $3.571(5)$ & N48 & N15 & $3.337(5)$ \\
\hline
\end{tabular}




\begin{tabular}{|c|c|c|c|c|c|}
\hline N48 & C20 & $3.343(6)$ & N48 & C21 & $3.539(6)$ \\
\hline C49 & C13 & $3.403(6)$ & C49 & C20 & $3.590(6)$ \\
\hline C49 & C21 & $3.261(6)$ & C50 & $\mathrm{Cg}$ & $3.394(6)$ \\
\hline C50 & N10 & $3.388(6)$ & C50 & C13 & $3.401(6)$ \\
\hline C50 & C5910 & $3.491(6)$ & C51 & $\mathrm{Cg}$ & $3.559(7)$ \\
\hline C51 & C5910 & $3.470(6)$ & N52 & N14 & $3.374(5)$ \\
\hline N52 & $\mathrm{Cl} 67$ & $3.085(3)$ & C53 & N14 & $3.390(6)$ \\
\hline C53 & N15 & $3.355(6)$ & C53 & C16 & $3.599(6)$ \\
\hline C54 & N15 & $3.568(6)$ & C54 & C16 & $3.289(6)$ \\
\hline C54 & N22 & $3.308(5)$ & N55 & C21 & $3.317(5)$ \\
\hline N55 & Cl68 & $3.326(4)$ & C56 & $\mathrm{F} 66^{3}$ & $3.424(5)$ \\
\hline O58 & $\mathrm{C} 36^{1}$ & $3.488(6)$ & O58 & Cl68 ${ }^{10}$ & $3.202(3)$ \\
\hline C59 & $\mathrm{C} 50^{11}$ & $3.491(6)$ & C59 & C5111 & $3.470(6)$ \\
\hline C62 & Cl67 & $3.595(4)$ & C63 & $\mathrm{N} 6^{12}$ & $3.588(6)$ \\
\hline C64 & F34 ${ }^{1}$ & $3.469(5)$ & C65 & F34 ${ }^{1}$ & $2.975(5)$ \\
\hline F66 & $\mathrm{N} 10^{9}$ & $3.489(5)$ & F66 & C12 ${ }^{9}$ & $3.350(6)$ \\
\hline F66 & $\mathrm{C} 56^{9}$ & $3.424(5)$ & Cl67 & N22 & $3.315(4)$ \\
\hline Cl67 & $O 25^{6}$ & $3.182(3)$ & $\mathrm{Cl} 67$ & N52 & $3.085(3)$ \\
\hline Cl67 & C62 & $3.595(4)$ & Cl68 & N19 & $3.094(3)$ \\
\hline Cl68 & N55 & $3.326(4)$ & Cl68 & $058^{11}$ & $3.202(3)$ \\
\hline
\end{tabular}

Symmetry Operators:
(1) $X+1, Y, Z$
(2) $-X+2, Y+1 / 2,-Z$
(3) $X, Y+1, Z$
(4) $-X+1, Y+1 / 2,-Z$
(5) $X-1, Y, Z$
(6) $-X+1, Y+1 / 2-1,-Z$
(7) $-X+1, Y+1 / 2,-Z+1$
(8) $-X+1, Y+1 / 2-1,-Z+1$
(9) $X, Y-1, Z$
(10) $-X+2, Y+1 / 2-1,-Z+1$
(11) $-X+2, Y+1 / 2,-Z+1$
(12) $-X+2, Y+1 / 2-1,-Z$ 
Table S14. Intermolecular contacts less than $3.60 \AA$ involving hydrogens

\begin{tabular}{|c|c|c|c|c|c|}
\hline atom & atom & distance & atom & atom & distance \\
\hline $\mathrm{F} 1$ & $\mathrm{H}_{2} 9 \mathrm{~A}^{1}$ & 2.690 & $\mathrm{~F} 1$ & $\mathrm{H} 29 \mathrm{~B}^{1}$ & 2.637 \\
\hline $\mathrm{F} 1$ & $\mathrm{H} 30^{1}$ & 3.078 & $\mathrm{C} 2$ & $\mathrm{H} 8 \mathrm{C}^{2}$ & 2.952 \\
\hline C3 & $\mathrm{H}_{8} \mathrm{~B}^{2}$ & 3.563 & C3 & $\mathrm{H} 8 \mathrm{C}^{2}$ & 3.241 \\
\hline C3 & $\mathrm{H} 25^{1}$ & 3.194 & C3 & $\mathrm{H}_{2} 6 \mathrm{~A}^{1}$ & 3.336 \\
\hline C4 & $\mathrm{H} 8 \mathrm{C}^{2}$ & 3.389 & C4 & $\mathrm{H} 62 \mathrm{~A}^{2}$ & 3.340 \\
\hline C4 & H65B & 3.455 & C5 & $\mathrm{H} 8 \mathrm{C}^{2}$ & 3.292 \\
\hline C5 & $\mathrm{H} 64 \mathrm{~A}$ & 3.504 & C5 & H65B & 2.772 \\
\hline N6 & $\mathrm{H} 63^{2}$ & 2.800 & N6 & $\mathrm{H} 64 \mathrm{~A}$ & 3.105 \\
\hline N6 & $\mathrm{H} 64 \mathrm{~A}^{2}$ & 3.161 & N6 & H65B & 2.989 \\
\hline C7 & $\mathrm{H} 63^{2}$ & 3.496 & C7 & $\mathrm{H} 64 \mathrm{~A}^{2}$ & 3.305 \\
\hline C7 & H65B & 3.178 & C8 & $\mathrm{H} 63^{2}$ & 3.547 \\
\hline C9 & $\mathrm{H} 50$ & 3.431 & $\mathrm{C9}$ & H65B & 3.280 \\
\hline N10 & $\mathrm{H} 50$ & 3.111 & N10 & H56B & 2.927 \\
\hline N10 & $\mathrm{H} 63^{3}$ & 2.991 & N10 & H65B & 3.170 \\
\hline C11 & $\mathrm{H} 8 \mathrm{C}^{2}$ & 2.994 & C11 & $\mathrm{H} 50$ & 3.566 \\
\hline C11 & H56B & 3.549 & C11 & $\mathrm{H} 63^{3}$ & 3.383 \\
\hline C11 & H65B & 2.870 & $\mathrm{C} 12$ & $\mathrm{H} 8 \mathrm{C}^{2}$ & 2.839 \\
\hline C12 & H56B & 3.445 & $\mathrm{C} 12$ & $\mathrm{H} 63^{3}$ & 3.347 \\
\hline C12 & $\mathrm{H} 65 \mathrm{~B}$ & 3.571 & N14 & $\mathrm{H} 26 \mathrm{~B}^{4}$ & 3.590 \\
\hline N14 & $\mathrm{H} 26 \mathrm{C}^{4}$ & 3.594 & N15 & $\mathrm{H} 26 \mathrm{~B}^{4}$ & 3.203 \\
\hline N15 & $\mathrm{H} 26 \mathrm{C}^{4}$ & 3.170 & C16 & $\mathrm{H} 26 \mathrm{~B}^{4}$ & 3.084 \\
\hline C16 & $\mathrm{H} 26 \mathrm{C}^{4}$ & 3.566 & C16 & $\mathrm{H} 27 \mathrm{~B}^{4}$ & 3.480 \\
\hline C16 & H54 & 3.499 & C17 & $\mathrm{H} 26 \mathrm{~B}^{4}$ & 3.506 \\
\hline C17 & $\mathrm{H} 27 \mathrm{~A}^{4}$ & 3.221 & C17 & $\mathrm{H} 27 \mathrm{~B}^{4}$ & 2.868 \\
\hline C18 & $\mathrm{H} 27 \mathrm{~A}^{4}$ & 2.827 & C18 & $\mathrm{H} 27 \mathrm{~B}^{4}$ & 3.171 \\
\hline C18 & $\mathrm{H} 45 \mathrm{C}$ & 3.404 & N19 & $\mathrm{H} 26 \mathrm{C}^{4}$ & 3.511 \\
\hline N19 & $\mathrm{H} 27 \mathrm{~A}^{4}$ & 3.224 & N19 & $\mathrm{H} 45 \mathrm{C}$ & 3.411 \\
\hline $\mathrm{C} 20$ & $\mathrm{H} 26 \mathrm{C}^{4}$ & 3.164 & $\mathrm{C} 21$ & $\mathrm{H} 26 \mathrm{C}^{4}$ & 3.573 \\
\hline C21 & H55 & 3.513 & $\mathrm{C} 21$ & H56B & 3.550 \\
\hline C21 & $\mathrm{H} 62 \mathrm{~B}^{3}$ & 3.250 & N22 & $\mathrm{H}^{26 \mathrm{~B}^{4}}$ & 3.411 \\
\hline N22 & H54 & 3.194 & $\mathrm{C} 23$ & H54 & 3.329 \\
\hline C24 & $H 3^{5}$ & 3.388 & $\mathrm{O} 25$ & $H 3^{5}$ & 2.553 \\
\hline $\mathrm{O} 25$ & $\mathrm{H}_{23} \mathrm{~B}^{4}$ & 3.489 & $\mathrm{O} 25$ & $\mathrm{H} 26 \mathrm{~B}^{4}$ & 3.514 \\
\hline $\mathrm{O} 25$ & $\mathrm{H}_{27 \mathrm{~B}^{4}}$ & 2.756 & $\mathrm{C} 26$ & $\mathrm{H} 3^{5}$ & 3.129 \\
\hline
\end{tabular}




\begin{tabular}{|c|c|c|c|c|c|}
\hline $\mathrm{C} 26$ & $\mathrm{H} 27 \mathrm{C}^{6}$ & 3.478 & $\mathrm{C} 27$ & $\mathrm{H} 17^{6}$ & 3.566 \\
\hline $\mathrm{C} 27$ & $\mathrm{H} 26 \mathrm{~B}^{4}$ & 3.411 & C27 & $\mathrm{H} 29 \mathrm{~B}^{6}$ & 3.027 \\
\hline N28 & $\mathrm{H} 27 \mathrm{~A}^{4}$ & 2.963 & N28 & $\mathrm{H} 27 \mathrm{~B}^{4}$ & 3.453 \\
\hline N28 & $\mathrm{H} 45 \mathrm{C}$ & 3.297 & C29 & $\mathrm{H} 27 \mathrm{~A}^{4}$ & 3.370 \\
\hline $\mathrm{C} 29$ & $\mathrm{H} 27 \mathrm{~B}^{4}$ & 3.389 & C30 & $\mathrm{H} 44^{3}$ & 3.382 \\
\hline C31 & $\mathrm{H} 44^{3}$ & 3.471 & C31 & $\mathrm{H} 54^{3}$ & 3.463 \\
\hline C32 & $\mathrm{H} 27 \mathrm{~A}^{4}$ & 3.450 & C32 & $\mathrm{H} 45 \mathrm{C}$ & $3.37 \varepsilon$ \\
\hline C32 & $\mathrm{H} 54^{3}$ & 2.995 & F33 & $\mathrm{H} 23 \mathrm{~B}^{3}$ & $3.41 \varepsilon$ \\
\hline F33 & $\mathrm{H} 26 \mathrm{~A}^{3}$ & 3.268 & F33 & $\mathrm{H} 26 \mathrm{~B}^{3}$ & 3.533 \\
\hline F33 & $\mathrm{H} 27 \mathrm{~A}^{4}$ & 3.400 & F33 & $\mathrm{H} 27 \mathrm{C}^{4}$ & 3.217 \\
\hline F33 & $\mathrm{H} 44^{3}$ & 2.673 & F34 & $\mathrm{H} 64 \mathrm{~B}^{5}$ & 3.022 \\
\hline F34 & $\mathrm{H} 65 \mathrm{~A}^{5}$ & 2.518 & F34 & $\mathrm{H} 65 \mathrm{~B}^{5}$ & $2.78 \subseteq$ \\
\hline $\mathrm{C} 35$ & $\mathrm{H} 45 \mathrm{~A}^{7}$ & 3.556 & C35 & $\mathrm{H} 45 \mathrm{~B}^{7}$ & 3.087 \\
\hline C36 & $\mathrm{H}_{45 \mathrm{~B}^{7}}$ & 3.222 & C36 & $\mathrm{H} 58^{5}$ & 3.474 \\
\hline C36 & $\mathrm{H} 60 \mathrm{~A}^{5}$ & 3.391 & C37 & $\mathrm{H} 29 \mathrm{~A}$ & 3.413 \\
\hline C37 & $\mathrm{H} 31 \mathrm{~A}^{7}$ & 3.570 & C37 & $\mathrm{H} 45 \mathrm{~B}^{7}$ & 3.242 \\
\hline C38 & $\mathrm{H} 29 \mathrm{~A}$ & 2.805 & C38 & $\mathrm{H} 31 \mathrm{~A}^{7}$ & 3.135 \\
\hline C38 & $\mathrm{H}_{4} 5 \mathrm{~B}^{7}$ & 3.116 & N39 & $\mathrm{H} 29 \mathrm{~A}$ & 2.850 \\
\hline N39 & $\mathrm{H} 31 \mathrm{~A}$ & 3.533 & N39 & $\mathrm{H} 31 \mathrm{~A}^{7}$ & $2.72 C$ \\
\hline N39 & $\mathrm{H} 31 \mathrm{~B}^{7}$ & 3.177 & $\mathrm{C} 40$ & $\mathrm{H} 29 \mathrm{~A}$ & 3.194 \\
\hline C40 & $\mathrm{H} 31 \mathrm{~A}^{7}$ & 3.146 & C41 & $\mathrm{H} 17$ & 3.346 \\
\hline C41 & $\mathrm{H} 29 \mathrm{~A}$ & 3.482 & N42 & $\mathrm{H} 17$ & 3.092 \\
\hline N42 & $\mathrm{H} 23 \mathrm{~A}$ & 2.881 & N42 & $\mathrm{H} 29 \mathrm{~A}$ & 3.464 \\
\hline N42 & $\mathrm{H} 1 \mathrm{~B}^{8}$ & 2.891 & C43 & $\mathrm{H} 17$ & 3.512 \\
\hline C43 & $\mathrm{H} 23 \mathrm{~A}$ & 3.468 & C43 & $\mathrm{H} 29 \mathrm{~A}$ & 3.115 \\
\hline $\mathrm{C} 43$ & $\mathrm{H}^{3} 1 \mathrm{~B}^{8}$ & 3.274 & $\mathrm{C} 43$ & $\mathrm{H} 45 \mathrm{~B}^{7}$ & 2.952 \\
\hline C44 & $\mathrm{H} 23 \mathrm{~A}$ & 3.420 & C44 & $\mathrm{H} 31 \mathrm{~B}^{8}$ & 3.156 \\
\hline C44 & $\mathrm{H} 45 \mathrm{~B}^{7}$ & 2.974 & $\mathrm{C} 45$ & $\mathrm{H} 32 \mathrm{~B}$ & 3.438 \\
\hline N48 & $\mathrm{H}^{2} \mathrm{BB}^{9}$ & 3.198 & N48 & $\mathrm{H} 60 \mathrm{C}^{9}$ & 3.180 \\
\hline $\mathrm{C} 49$ & $\mathrm{H} 21$ & 3.436 & C49 & $\mathrm{H}^{2} \mathrm{~B}^{9}$ & $3.56 s$ \\
\hline C49 & $\mathrm{H} 60 \mathrm{C}^{9}$ & 3.020 & C50 & $\mathrm{H} 59 \mathrm{~A}^{9}$ & 3.150 \\
\hline C50 & $\mathrm{H} 59 \mathrm{C}^{9}$ & 2.956 & C50 & $\mathrm{H} 60 \mathrm{C}^{9}$ & 3.384 \\
\hline C51 & $\mathrm{H} 59 \mathrm{~A}^{9}$ & 2.790 & C51 & $\mathrm{H} 59 \mathrm{C}^{9}$ & $3.27 \varepsilon$ \\
\hline N52 & $\mathrm{H} 59 \mathrm{~A}^{9}$ & 3.240 & N52 & $\mathrm{H} 60 \mathrm{~B}^{9}$ & 3.492 \\
\hline C53 & $\mathrm{H}^{6} \mathrm{BB}^{9}$ & 3.167 & C54 & $\mathrm{H} 22$ & 3.501 \\
\hline C54 & $\mathrm{H} 23 \mathrm{~A}$ & 3.492 & C54 & $\mathrm{H} 32 \mathrm{~A}^{8}$ & 3.205 \\
\hline N55 & $\mathrm{H} 21$ & 3.164 & N55 & $\mathrm{H} 60 \mathrm{C}^{9}$ & 3.345 \\
\hline C56 & $\mathrm{H} 21$ & 3.336 & C57 & $\mathrm{H} 36^{1}$ & 3.423 \\
\hline
\end{tabular}




\begin{tabular}{|c|c|c|c|c|c|}
\hline O58 & $\mathrm{H} 36^{1}$ & 2.568 & O58 & $\mathrm{H}_{56 \mathrm{~A}^{9}}$ & 3.423 \\
\hline O58 & $\mathrm{H}_{59 \mathrm{C}^{9}}$ & 2.721 & O58 & $\mathrm{H} 60 \mathrm{C}^{9}$ & 3.380 \\
\hline C59 & $\mathrm{H} 50^{10}$ & 3.521 & C59 & $\mathrm{H} 58^{10}$ & 3.553 \\
\hline C59 & $\mathrm{H} 60 \mathrm{C}^{9}$ & 3.382 & C59 & $\mathrm{H} 65 \mathrm{~A}^{10}$ & 3.135 \\
\hline C60 & $\mathrm{H} 36^{1}$ & 3.196 & $\mathrm{C} 60$ & $\mathrm{H} 37^{1}$ & 3.542 \\
\hline C60 & $H 59 B^{10}$ & 3.402 & N61 & $\mathrm{H} 8 \mathrm{~B}$ & 3.443 \\
\hline N61 & $\mathrm{H} 59 \mathrm{~A}^{9}$ & 2.948 & N61 & $\mathrm{H}_{59 \mathrm{C}^{9}}$ & 3.538 \\
\hline C62 & H8B & 3.595 & C62 & $\mathrm{H} 21^{8}$ & 3.034 \\
\hline C62 & $\mathrm{H}_{59} \mathrm{~A}^{9}$ & 3.377 & C63 & $\mathrm{H} 12^{8}$ & 3.258 \\
\hline C63 & $\mathrm{H} 21^{8}$ & 3.254 & C65 & $\mathrm{H}_{59 \mathrm{~A}^{9}}$ & 3.430 \\
\hline C65 & $\mathrm{H} 59 \mathrm{C}^{9}$ & 3.508 & F66 & $\mathrm{H} 12^{8}$ & 2.561 \\
\hline F66 & $\mathrm{H} 21^{8}$ & 3.246 & F66 & $\mathrm{H} 56 \mathrm{~A}^{8}$ & 2.888 \\
\hline F66 & $\mathrm{H} 56 \mathrm{~B}^{8}$ & 3.032 & F66 & $\mathrm{H}_{59 \mathrm{~A}^{9}}$ & 3.274 \\
\hline F66 & $\mathrm{H}^{2} 9 \mathrm{~B}^{9}$ & 3.378 & F66 & $\mathrm{H} 60 \mathrm{~A}^{8}$ & 3.382 \\
\hline $\mathrm{Cl} 67$ & $\mathrm{H} 3^{11}$ & 3.554 & $\mathrm{Cl} 67$ & H8B & 3.266 \\
\hline $\mathrm{Cl} 67$ & $\mathrm{H} 19^{8}$ & 3.468 & $\mathrm{Cl} 67$ & $\mathrm{H} 22$ & 2.717 \\
\hline $\mathrm{Cl} 67$ & $\mathrm{H} 23 \mathrm{~B}$ & 2.973 & $\mathrm{Cl} 67$ & $\mathrm{H} 25^{6}$ & 2.380 \\
\hline $\mathrm{Cl} 67$ & $\mathrm{H} 26 \mathrm{C}^{6}$ & 3.283 & $\mathrm{Cl} 67$ & $\mathrm{H} 27 \mathrm{~A}^{6}$ & 3.063 \\
\hline $\mathrm{Cl} 67$ & $\mathrm{H} 32 \mathrm{~A}^{8}$ & 3.262 & $\mathrm{Cl} 67$ & H52 & 2.351 \\
\hline $\mathrm{Cl} 67$ & H54 & 3.385 & $\mathrm{Cl} 67$ & $\mathrm{H} 62 \mathrm{~A}$ & 2.954 \\
\hline Cl67 & H62B & 3.459 & Cl68 & $\mathrm{H} 19$ & 2.371 \\
\hline Cl68 & $\mathrm{H} 21$ & 3.298 & Cl68 & H32B & 2.774 \\
\hline Cl68 & $\mathrm{H} 45 \mathrm{C}$ & 3.192 & Cl68 & $\mathrm{H} 52^{3}$ & 3.451 \\
\hline Cl68 & H55 & 2.753 & Cl68 & $\mathrm{H} 56 \mathrm{~A}$ & 2.969 \\
\hline Cl68 & $\mathrm{H} 58^{10}$ & 2.400 & $\mathrm{Cl} 68$ & $H 59 A^{10}$ & 3.116 \\
\hline $\mathrm{Cl} 68$ & $\mathrm{H}^{2} \mathrm{~B}^{10}$ & 3.224 & $\mathrm{Cl} 68$ & $\mathrm{H} 62 \mathrm{~B}^{3}$ & 3.090 \\
\hline $\mathrm{H} 3$ & $\mathrm{C} 24^{1}$ & 3.388 & $\mathrm{H} 3$ & $\mathrm{O} 25^{1}$ & 2.553 \\
\hline $\mathrm{H} 3$ & $\mathrm{C} 26^{1}$ & 3.129 & $\mathrm{H} 3$ & $\mathrm{Cl} 67^{2}$ & 3.554 \\
\hline $\mathrm{H} 3$ & $\mathrm{H} 25^{1}$ & 2.335 & $\mathrm{H} 3$ & $\mathrm{H}_{26 \mathrm{~A}^{1}}$ & 2.680 \\
\hline $\mathrm{H} 3$ & $\mathrm{H} 26 \mathrm{C}^{1}$ & 2.991 & $\mathrm{H} 4$ & $\mathrm{H} 26 \mathrm{~A}^{1}$ & 3.287 \\
\hline $\mathrm{H} 4$ & $\mathrm{H} 26 \mathrm{C}^{1}$ & 3.027 & $\mathrm{H} 4$ & $\mathrm{H} 62 \mathrm{~A}^{2}$ & 2.978 \\
\hline $\mathrm{H} 4$ & $\mathrm{H}_{6} 3^{2}$ & 3.373 & $\mathrm{H} 8 \mathrm{~A}$ & $\mathrm{H} 64 \mathrm{~A}^{2}$ & 3.361 \\
\hline H8A & $\mathrm{H}_{64 \mathrm{~B}^{2}}$ & 3.286 & $\mathrm{H} 8 \mathrm{~B}$ & $C 3^{11}$ & 3.563 \\
\hline $\mathrm{H} 8 \mathrm{~B}$ & N61 & 3.443 & $\mathrm{H} 8 \mathrm{~B}$ & C62 & 3.595 \\
\hline H8B & $\mathrm{Cl} 67$ & 3.266 & $\mathrm{H} 8 \mathrm{~B}$ & H52 & 3.491 \\
\hline $\mathrm{H} 8 \mathrm{~B}$ & $\mathrm{H} 62 \mathrm{~A}$ & 2.905 & $\mathrm{H} 8 \mathrm{C}$ & $C 2^{11}$ & 2.952 \\
\hline $\mathrm{H} 8 \mathrm{C}$ & $C 3^{11}$ & 3.241 & $\mathrm{H} 8 \mathrm{C}$ & $C 4^{11}$ & 3.389 \\
\hline $\mathrm{H} 8 \mathrm{C}$ & $C 5^{11}$ & 3.292 & $\mathrm{H} 8 \mathrm{C}$ & C1111 & 2.994 \\
\hline
\end{tabular}




\begin{tabular}{|c|c|c|c|c|c|}
\hline $\mathrm{H} 8 \mathrm{C}$ & $\mathrm{C} 12^{11}$ & 2.839 & $\mathrm{H} 8 \mathrm{C}$ & $\mathrm{H} 12^{11}$ & 3.224 \\
\hline $\mathrm{H} 8 \mathrm{C}$ & $\mathrm{H}_{6} 3^{2}$ & 2.938 & $\mathrm{H} 8 \mathrm{C}$ & $\mathrm{H} 64 \mathrm{~A}^{2}$ & 3.564 \\
\hline $\mathrm{H} 8 \mathrm{C}$ & $\mathrm{H}_{64 \mathrm{~B}^{2}}$ & 3.500 & $\mathrm{H} 12$ & $\mathrm{C} 63^{3}$ & 3.258 \\
\hline $\mathrm{H} 12$ & $\mathrm{~F} 66^{3}$ & 2.561 & $\mathrm{H} 12$ & $\mathrm{H} 8 \mathrm{C}^{2}$ & 3.224 \\
\hline $\mathrm{H} 12$ & H56B & 2.896 & $\mathrm{H} 12$ & $\mathrm{H} 60 \mathrm{~A}$ & 3.122 \\
\hline $\mathrm{H} 12$ & $\mathrm{H} 63^{3}$ & 3.020 & $\mathrm{H} 12$ & $\mathrm{H}^{2} 4 \mathrm{~B}^{3}$ & 3.569 \\
\hline $\mathrm{H} 17$ & $\mathrm{C} 27^{4}$ & 3.566 & $\mathrm{H} 17$ & C41 & 3.346 \\
\hline $\mathrm{H} 17$ & N42 & 3.092 & $\mathrm{H} 17$ & C43 & 3.512 \\
\hline $\mathrm{H} 17$ & $\mathrm{H} 27 \mathrm{~A}^{4}$ & 3.482 & $\mathrm{H} 17$ & $\mathrm{H} 27 \mathrm{~B}^{4}$ & 2.802 \\
\hline H19 & $\mathrm{Cl} 67^{3}$ & 3.468 & H19 & Cl68 & 2.371 \\
\hline H19 & $\mathrm{H} 27 \mathrm{~A}^{4}$ & 3.490 & $\mathrm{H} 19$ & $\mathrm{H} 45 \mathrm{C}$ & 3.265 \\
\hline H19 & $\mathrm{H} 62 \mathrm{~B}^{3}$ & 3.525 & $\mathrm{H} 21$ & C49 & 3.436 \\
\hline $\mathrm{H} 21$ & N55 & 3.164 & $\mathrm{H} 21$ & C56 & 3.336 \\
\hline $\mathrm{H} 21$ & $\mathrm{C} 62^{3}$ & 3.034 & $\mathrm{H} 21$ & $\mathrm{C} 63^{3}$ & 3.254 \\
\hline $\mathrm{H} 21$ & F66 ${ }^{3}$ & 3.246 & $\mathrm{H} 21$ & Cl68 & 3.298 \\
\hline $\mathrm{H} 21$ & H55 & 3.369 & $\mathrm{H} 21$ & $\mathrm{H} 56 \mathrm{~A}$ & 3.159 \\
\hline $\mathrm{H} 21$ & H56B & 3.078 & $\mathrm{H} 21$ & $\mathrm{H} 62 \mathrm{~A}^{3}$ & 3.170 \\
\hline $\mathrm{H} 21$ & $\mathrm{H}_{62} \mathrm{~B}^{3}$ & 2.420 & $\mathrm{H} 21$ & $\mathrm{H} 63^{3}$ & 3.071 \\
\hline H22 & C54 & 3.501 & $\mathrm{H} 22$ & $\mathrm{Cl} 67$ & 2.717 \\
\hline H22 & $\mathrm{H}_{26 \mathrm{~B}^{4}}$ & 3.536 & $\mathrm{H} 22$ & H52 & 3.591 \\
\hline $\mathrm{H} 22$ & H54 & 3.395 & $\mathrm{H} 23 \mathrm{~A}$ & N42 & 2.881 \\
\hline $\mathrm{H} 23 \mathrm{~A}$ & C43 & 3.468 & $\mathrm{H} 23 \mathrm{~A}$ & C44 & 3.420 \\
\hline $\mathrm{H} 23 \mathrm{~A}$ & C54 & 3.492 & $\mathrm{H} 23 \mathrm{~A}$ & $\mathrm{H} 44$ & 2.930 \\
\hline $\mathrm{H} 23 \mathrm{~A}$ & H54 & 3.043 & $\mathrm{H} 23 \mathrm{~B}$ & $\mathrm{O} 25^{6}$ & 3.489 \\
\hline $\mathrm{H} 23 \mathrm{~B}$ & F33 ${ }^{8}$ & 3.418 & $\mathrm{H} 23 \mathrm{~B}$ & $\mathrm{Cl} 67$ & 2.973 \\
\hline $\mathrm{H} 23 \mathrm{~B}$ & $\mathrm{H} 25^{6}$ & 3.177 & $\mathrm{H} 23 \mathrm{~B}$ & $\mathrm{H} 27 \mathrm{~A}^{6}$ & 3.230 \\
\hline $\mathrm{H} 23 \mathrm{~B}$ & $\mathrm{H} 27 \mathrm{C}^{6}$ & 3.259 & $\mathrm{H} 23 \mathrm{~B}$ & $\mathrm{H} 32 \mathrm{~A}^{8}$ & 3.283 \\
\hline $\mathrm{H} 23 \mathrm{~B}$ & H54 & 3.158 & $\mathrm{H} 25$ & $C 3^{5}$ & 3.194 \\
\hline $\mathrm{H} 25$ & $\mathrm{Cl} 67^{4}$ & 2.380 & $\mathrm{H} 25$ & $\mathrm{H} 3^{5}$ & 2.335 \\
\hline $\mathrm{H} 25$ & $\mathrm{H}_{2} 3 \mathrm{~B}^{4}$ & 3.177 & $\mathrm{H} 25$ & $\mathrm{H}_{27 \mathrm{~B}^{4}}$ & 2.870 \\
\hline $\mathrm{H} 26 \mathrm{~A}$ & $C 3^{5}$ & 3.336 & $\mathrm{H} 26 \mathrm{~A}$ & F33 ${ }^{8}$ & 3.268 \\
\hline $\mathrm{H} 26 \mathrm{~A}$ & $\mathrm{H} 3^{5}$ & 2.680 & $\mathrm{H} 26 \mathrm{~A}$ & $\mathrm{H} 4^{5}$ & 3.287 \\
\hline $\mathrm{H} 26 \mathrm{~A}$ & $\mathrm{H} 44$ & 3.076 & $\mathrm{H} 26 \mathrm{~B}$ & $\mathrm{~N} 14^{6}$ & 3.590 \\
\hline H26B & N15 ${ }^{6}$ & 3.203 & $\mathrm{H} 26 \mathrm{~B}$ & $\mathrm{C} 16^{6}$ & 3.084 \\
\hline H26B & $\mathrm{C} 17^{6}$ & 3.506 & $\mathrm{H} 26 \mathrm{~B}$ & $\mathrm{~N} 22^{6}$ & 3.411 \\
\hline H26B & $\mathrm{O} 25^{6}$ & 3.514 & $\mathrm{H} 26 \mathrm{~B}$ & $\mathrm{C} 27^{6}$ & 3.411 \\
\hline H26B & F33 ${ }^{8}$ & 3.533 & $\mathrm{H} 26 \mathrm{~B}$ & $\mathrm{H} 22^{6}$ & 3.536 \\
\hline H26B & $\mathrm{H} 27 \mathrm{C}^{6}$ & 2.553 & $\mathrm{H} 26 \mathrm{C}$ & $\mathrm{N} 14^{6}$ & 3.594 \\
\hline
\end{tabular}




\begin{tabular}{|c|c|c|c|c|c|}
\hline $\mathrm{H} 26 \mathrm{C}$ & N15 ${ }^{6}$ & 3.170 & $\mathrm{H} 26 \mathrm{C}$ & $\mathrm{C} 16^{6}$ & 3.566 \\
\hline $\mathrm{H} 26 \mathrm{C}$ & N196 & 3.511 & $\mathrm{H} 26 \mathrm{C}$ & $\mathrm{C} 20^{6}$ & 3.164 \\
\hline $\mathrm{H} 26 \mathrm{C}$ & $\mathrm{C} 21^{6}$ & 3.573 & $\mathrm{H} 26 \mathrm{C}$ & $\mathrm{Cl} 67^{4}$ & 3.283 \\
\hline $\mathrm{H} 26 \mathrm{C}$ & $\mathrm{H} 3^{5}$ & 2.991 & $\mathrm{H} 26 \mathrm{C}$ & $\mathrm{H} 4^{5}$ & 3.027 \\
\hline $\mathrm{H} 27 \mathrm{~A}$ & $\mathrm{C} 17^{6}$ & 3.221 & $\mathrm{H} 27 \mathrm{~A}$ & $\mathrm{C} 18^{6}$ & 2.827 \\
\hline $\mathrm{H} 27 \mathrm{~A}$ & N196 & 3.224 & $\mathrm{H} 27 \mathrm{~A}$ & $\mathrm{~N} 28^{6}$ & 2.963 \\
\hline $\mathrm{H} 27 \mathrm{~A}$ & $\mathrm{C} 29^{6}$ & 3.370 & $\mathrm{H} 27 \mathrm{~A}$ & $\mathrm{C} 32^{6}$ & 3.450 \\
\hline $\mathrm{H} 27 \mathrm{~A}$ & F33 ${ }^{6}$ & 3.400 & $\mathrm{H} 27 \mathrm{~A}$ & $\mathrm{Cl} 67^{4}$ & 3.063 \\
\hline $\mathrm{H} 27 \mathrm{~A}$ & $\mathrm{H} 17^{6}$ & 3.482 & $\mathrm{H} 27 \mathrm{~A}$ & $\mathrm{H} 19^{6}$ & 3.490 \\
\hline $\mathrm{H} 27 \mathrm{~A}$ & $\mathrm{H} 23 \mathrm{~B}^{4}$ & 3.230 & $\mathrm{H} 27 \mathrm{~A}$ & $\mathrm{H} 29 \mathrm{~B}^{6}$ & 2.919 \\
\hline $\mathrm{H} 27 \mathrm{~A}$ & $\mathrm{H} 32 \mathrm{~A}^{6}$ & 2.969 & $\mathrm{H} 27 \mathrm{~B}$ & $\mathrm{C} 16^{6}$ & 3.480 \\
\hline H27B & $\mathrm{C} 17^{6}$ & 2.868 & $\mathrm{H} 27 \mathrm{~B}$ & $\mathrm{C} 18^{6}$ & 3.171 \\
\hline H27B & $\mathrm{O} 25^{6}$ & 2.756 & $\mathrm{H} 27 \mathrm{~B}$ & $\mathrm{~N} 28^{6}$ & 3.453 \\
\hline H27B & $C 29^{6}$ & 3.389 & H27B & $\mathrm{H} 17^{6}$ & 2.802 \\
\hline $\mathrm{H} 27 \mathrm{~B}$ & $\mathrm{H} 25^{6}$ & 2.870 & $\mathrm{H} 27 \mathrm{~B}$ & $\mathrm{H}_{29 \mathrm{~B}^{6}}$ & 2.605 \\
\hline $\mathrm{H} 27 \mathrm{C}$ & $\mathrm{C} 26^{4}$ & 3.478 & $\mathrm{H} 27 \mathrm{C}$ & F336 & 3.217 \\
\hline $\mathrm{H} 27 \mathrm{C}$ & $\mathrm{H} 23 \mathrm{~B}^{4}$ & 3.259 & $\mathrm{H} 27 \mathrm{C}$ & $\mathrm{H} 26 \mathrm{~B}^{4}$ & 2.553 \\
\hline $\mathrm{H} 27 \mathrm{C}$ & $\mathrm{H}_{2} 9 \mathrm{~B}^{6}$ & 3.061 & $\mathrm{H} 29 \mathrm{~A}$ & $F 1^{5}$ & 2.690 \\
\hline $\mathrm{H} 29 \mathrm{~A}$ & C37 & 3.413 & $\mathrm{H} 29 \mathrm{~A}$ & $\mathrm{C} 38$ & 2.805 \\
\hline $\mathrm{H} 29 \mathrm{~A}$ & N39 & 2.850 & $\mathrm{H} 29 \mathrm{~A}$ & $\mathrm{C} 40$ & 3.194 \\
\hline $\mathrm{H} 29 \mathrm{~A}$ & C41 & 3.482 & $\mathrm{H} 29 \mathrm{~A}$ & N42 & 3.464 \\
\hline $\mathrm{H} 29 \mathrm{~A}$ & C43 & 3.115 & $\mathrm{H} 29 \mathrm{~B}$ & $\mathrm{~F} 1^{5}$ & 2.637 \\
\hline $\mathrm{H} 29 \mathrm{~B}$ & $\mathrm{C} 27^{4}$ & 3.027 & $\mathrm{H} 29 \mathrm{~B}$ & $\mathrm{H} 27 \mathrm{~A}^{4}$ & 2.919 \\
\hline $\mathrm{H} 29 \mathrm{~B}$ & $\mathrm{H} 27 \mathrm{~B}^{4}$ & 2.605 & $\mathrm{H} 29 \mathrm{~B}$ & $\mathrm{H} 27 \mathrm{C}^{4}$ & 3.061 \\
\hline H30 & $\mathrm{F} 1^{5}$ & 3.078 & $\mathrm{H} 30$ & $\mathrm{H} 44^{3}$ & 3.461 \\
\hline $\mathrm{H} 30$ & $\mathrm{H} 45 \mathrm{~A}^{12}$ & 3.373 & $\mathrm{H} 31 \mathrm{~A}$ & $\mathrm{C} 37^{12}$ & 3.570 \\
\hline $\mathrm{H} 31 \mathrm{~A}$ & C38 & 3.135 & $\mathrm{H} 31 \mathrm{~A}$ & N39 & 3.533 \\
\hline $\mathrm{H} 31 \mathrm{~A}$ & N3912 & 2.720 & $\mathrm{H} 31 \mathrm{~A}$ & $\mathrm{C} 40^{12}$ & 3.146 \\
\hline $\mathrm{H} 31 \mathrm{~A}$ & $\mathrm{H} 37^{12}$ & 3.533 & $\mathrm{H} 31 \mathrm{~A}$ & $\mathrm{H} 45 \mathrm{~A}^{12}$ & 3.525 \\
\hline $\mathrm{H} 31 \mathrm{~A}$ & $\mathrm{H} 45 \mathrm{~B}$ & 3.208 & $\mathrm{H} 31 \mathrm{~A}$ & $\mathrm{H} 45 \mathrm{~B}^{12}$ & 3.553 \\
\hline $\mathrm{H} 31 \mathrm{~A}$ & $\mathrm{H} 45 \mathrm{C}$ & 3.542 & H31B & $\mathrm{N} 39^{12}$ & 3.177 \\
\hline H31B & $\mathrm{N} 42^{3}$ & 2.891 & H31B & $\mathrm{C} 43^{3}$ & 3.274 \\
\hline H31B & $\mathrm{C} 44^{3}$ & 3.156 & H31B & $\mathrm{H} 44^{3}$ & 2.758 \\
\hline H31B & $\mathrm{H} 45 \mathrm{~B}^{12}$ & 3.233 & H31B & $\mathrm{H} 54^{3}$ & 2.957 \\
\hline $\mathrm{H} 32 \mathrm{~A}$ & $\mathrm{C} 54^{3}$ & 3.205 & $\mathrm{H} 32 \mathrm{~A}$ & $\mathrm{Cl} \mid 67^{3}$ & 3.262 \\
\hline $\mathrm{H} 32 \mathrm{~A}$ & $\mathrm{H} 23 \mathrm{~B}^{3}$ & 3.283 & $\mathrm{H} 32 \mathrm{~A}$ & $\mathrm{H} 27 \mathrm{~A}^{4}$ & 2.969 \\
\hline $\mathrm{H} 32 \mathrm{~A}$ & $\mathrm{H} 54^{3}$ & 2.327 & $\mathrm{H} 32 \mathrm{~B}$ & C45 & 3.439 \\
\hline H32B & $\mathrm{Cl} 68$ & 2.774 & H32B & $\mathrm{H} 37^{12}$ & 3.384 \\
\hline
\end{tabular}




\begin{tabular}{|c|c|c|c|c|c|}
\hline H32B & $\mathrm{H} 45 \mathrm{~B}$ & 3.407 & $\mathrm{H} 32 \mathrm{~B}$ & $\mathrm{H} 45 \mathrm{C}$ & 2.732 \\
\hline H32B & $\mathrm{H} 54^{3}$ & 3.062 & $\mathrm{H} 36$ & $\mathrm{C} 57^{5}$ & 3.423 \\
\hline H36 & $058^{5}$ & 2.568 & $\mathrm{H} 36$ & $\mathrm{C} 60^{5}$ & 3.196 \\
\hline H36 & $\mathrm{H} 58^{5}$ & 2.621 & $\mathrm{H} 36$ & $\mathrm{H} 60 \mathrm{~A}^{5}$ & 2.756 \\
\hline H36 & $\mathrm{H}^{6} 6 \mathrm{~B}^{5}$ & 3.056 & $\mathrm{H} 37$ & $\mathrm{C} 60^{5}$ & 3.542 \\
\hline H37 & $\mathrm{H} 31 \mathrm{~A}^{7}$ & 3.533 & H37 & $\mathrm{H} 32 \mathrm{~B}^{7}$ & 3.384 \\
\hline H37 & $\mathrm{H}_{60} \mathrm{~A}^{5}$ & 3.181 & $\mathrm{H} 37$ & $\mathrm{H}^{6} \mathrm{BB}^{5}$ & 3.004 \\
\hline $\mathrm{H} 44$ & $\mathrm{C} 30^{8}$ & 3.382 & $\mathrm{H} 44$ & $\mathrm{C} 31^{8}$ & 3.471 \\
\hline $\mathrm{H} 44$ & $\mathrm{~F}^{3} 3^{8}$ & 2.673 & $\mathrm{H} 44$ & $\mathrm{H} 23 \mathrm{~A}$ & 2.930 \\
\hline $\mathrm{H} 44$ & $\mathrm{H} 26 \mathrm{~A}$ & 3.076 & $\mathrm{H} 44$ & $\mathrm{H} 30^{8}$ & 3.461 \\
\hline $\mathrm{H} 44$ & H31B ${ }^{8}$ & 2.758 & $\mathrm{H} 44$ & $\mathrm{H} 45 \mathrm{~B}^{7}$ & 3.436 \\
\hline $\mathrm{H} 45 \mathrm{~A}$ & $\mathrm{C} 35^{12}$ & 3.556 & $\mathrm{H} 45 \mathrm{~A}$ & $\mathrm{H} 30^{7}$ & 3.373 \\
\hline $\mathrm{H} 45 \mathrm{~A}$ & $\mathrm{H} 31 \mathrm{~A}^{7}$ & 3.525 & $\mathrm{H} 45 \mathrm{~B}$ & $\mathrm{C} 35^{12}$ & 3.087 \\
\hline $\mathrm{H} 45 \mathrm{~B}$ & $\mathrm{C} 36^{12}$ & 3.222 & $\mathrm{H} 45 \mathrm{~B}$ & $\mathrm{C} 37^{12}$ & 3.242 \\
\hline $\mathrm{H} 45 \mathrm{~B}$ & $\mathrm{C} 38^{12}$ & 3.116 & $\mathrm{H} 45 \mathrm{~B}$ & $\mathrm{C} 43^{12}$ & 2.952 \\
\hline $\mathrm{H} 45 \mathrm{~B}$ & C44 ${ }^{12}$ & 2.974 & $\mathrm{H} 45 \mathrm{~B}$ & $\mathrm{H} 31 \mathrm{~A}$ & 3.208 \\
\hline $\mathrm{H} 45 \mathrm{~B}$ & $\mathrm{H} 31 \mathrm{~A}^{7}$ & 3.553 & $\mathrm{H} 45 \mathrm{~B}$ & $\mathrm{H} 31 \mathrm{~B}^{7}$ & 3.233 \\
\hline $\mathrm{H} 45 \mathrm{~B}$ & H32B & 3.407 & $\mathrm{H} 45 \mathrm{~B}$ & $\mathrm{H} 44^{12}$ & 3.436 \\
\hline $\mathrm{H} 45 \mathrm{C}$ & C18 & 3.404 & $\mathrm{H} 45 \mathrm{C}$ & N19 & 3.411 \\
\hline $\mathrm{H} 45 \mathrm{C}$ & N28 & 3.297 & $\mathrm{H} 45 \mathrm{C}$ & C32 & 3.378 \\
\hline $\mathrm{H} 45 \mathrm{C}$ & Cl68 & 3.192 & $\mathrm{H} 45 \mathrm{C}$ & $\mathrm{H} 19$ & 3.265 \\
\hline $\mathrm{H} 45 \mathrm{C}$ & H31A & 3.542 & $\mathrm{H} 45 \mathrm{C}$ & H32B & 2.732 \\
\hline $\mathrm{H} 50$ & C9 & 3.431 & $\mathrm{H} 50$ & N10 & 3.111 \\
\hline $\mathrm{H} 50$ & C11 & 3.566 & $\mathrm{H} 50$ & C599 & 3.521 \\
\hline $\mathrm{H} 50$ & $\mathrm{H} 59 \mathrm{~A}^{9}$ & 3.363 & $\mathrm{H} 50$ & $\mathrm{H} 59 \mathrm{C}^{9}$ & 2.811 \\
\hline H52 & $\mathrm{Cl} 67$ & 2.351 & H52 & $\mathrm{Cl} 68^{8}$ & 3.451 \\
\hline H52 & H8B & 3.491 & H52 & $\mathrm{H} 22$ & 3.591 \\
\hline H52 & $\mathrm{H} 59 \mathrm{~A}^{9}$ & 3.526 & $\mathrm{H} 54$ & C16 & 3.499 \\
\hline H54 & N22 & 3.194 & H54 & $\mathrm{C} 23$ & 3.329 \\
\hline H54 & $\mathrm{C} 31^{8}$ & 3.463 & H54 & $\mathrm{C} 32^{8}$ & 2.995 \\
\hline H54 & $\mathrm{Cl} 67$ & 3.385 & H54 & $\mathrm{H} 22$ & 3.395 \\
\hline H54 & $\mathrm{H} 23 \mathrm{~A}$ & 3.043 & H54 & $\mathrm{H} 23 \mathrm{~B}$ & 3.158 \\
\hline H54 & H31B & 2.957 & H54 & $\mathrm{H} 32 \mathrm{~A}^{8}$ & 2.327 \\
\hline H54 & $\mathrm{H} 32 \mathrm{~B}^{8}$ & 3.062 & H55 & $\mathrm{C} 21$ & 3.513 \\
\hline H55 & Cl68 & 2.753 & H55 & $\mathrm{H} 21$ & 3.369 \\
\hline H55 & $\mathrm{H} 58^{10}$ & 3.463 & H55 & $\mathrm{H} 60 \mathrm{C}^{9}$ & 3.501 \\
\hline H56A & $058^{10}$ & 3.423 & $\mathrm{H} 56 \mathrm{~A}$ & $\mathrm{~F} 66^{3}$ & 2.888 \\
\hline H56A & Cl68 & 2.969 & $\mathrm{H} 56 \mathrm{~A}$ & $\mathrm{H} 21$ & 3.159 \\
\hline
\end{tabular}




\begin{tabular}{|c|c|c|c|c|c|}
\hline $\mathrm{H} 56 \mathrm{~A}$ & $\mathrm{H} 58^{10}$ & 2.875 & $\mathrm{H} 56 \mathrm{~A}$ & $\mathrm{H}_{59} \mathrm{~A}^{10}$ & 3.287 \\
\hline $\mathrm{H} 56 \mathrm{~A}$ & $H 59 B^{10}$ & 3.185 & $\mathrm{H} 56 \mathrm{~A}$ & $\mathrm{H} 62 \mathrm{~B}^{3}$ & 3.511 \\
\hline H56B & N10 & 2.927 & $\mathrm{H} 56 \mathrm{~B}$ & C11 & 3.549 \\
\hline H56B & C12 & 3.445 & H56B & $\mathrm{C} 21$ & 3.550 \\
\hline H56B & $\mathrm{F} 66^{3}$ & 3.032 & H56B & $\mathrm{H} 12$ & 2.896 \\
\hline H56B & $\mathrm{H} 21$ & 3.078 & H58 & $\mathrm{C} 36^{1}$ & 3.474 \\
\hline H58 & C59 ${ }^{9}$ & 3.553 & H58 & $\mathrm{Cl} 168^{9}$ & 2.400 \\
\hline H58 & $\mathrm{H} 36^{1}$ & 2.621 & H58 & $\mathrm{H} 55^{9}$ & 3.463 \\
\hline H58 & $\mathrm{H} 6 \mathrm{~A}^{9}$ & 2.875 & $\mathrm{H} 58$ & $\mathrm{H}_{59 \mathrm{C}^{9}}$ & 2.662 \\
\hline H58 & $\mathrm{H} 60 \mathrm{C}^{9}$ & 3.404 & $\mathrm{H} 59 \mathrm{~A}$ & $\mathrm{C} 50^{10}$ & 3.150 \\
\hline H59A & C5 $51^{10}$ & 2.790 & H59A & N52 $2^{10}$ & 3.240 \\
\hline H59A & $\mathrm{N} 61^{10}$ & 2.948 & H59A & $\mathrm{C} 62^{10}$ & 3.377 \\
\hline $\mathrm{H} 59 \mathrm{~A}$ & $\mathrm{C} 65^{10}$ & 3.430 & $\mathrm{H} 59 \mathrm{~A}$ & F66 $6^{10}$ & 3.274 \\
\hline H59A & $\mathrm{Cl} 168^{9}$ & 3.116 & $\mathrm{H} 59 \mathrm{~A}$ & $\mathrm{H} 50^{10}$ & 3.363 \\
\hline H59A & $\mathrm{H} 52^{10}$ & 3.526 & H59A & $\mathrm{H} 56 \mathrm{~A}^{9}$ & 3.287 \\
\hline $\mathrm{H} 59 \mathrm{~A}$ & $\mathrm{H} 62 \mathrm{~B}^{10}$ & 2.968 & H59A & $H 65 A^{10}$ & 2.951 \\
\hline H59B & $\mathrm{C} 60^{9}$ & 3.402 & H59B & $\mathrm{F} 66^{10}$ & 3.378 \\
\hline H59B & $\mathrm{H}_{56} \mathrm{~A}^{9}$ & 3.185 & H59B & $\mathrm{H} 60 \mathrm{~A}^{9}$ & 3.574 \\
\hline H59B & $\mathrm{H} 60 \mathrm{C}^{9}$ & 2.511 & H59B & $\mathrm{H} 65 \mathrm{~A}^{10}$ & 3.172 \\
\hline H59C & $\mathrm{C} 50^{10}$ & 2.956 & $\mathrm{H} 59 \mathrm{C}$ & C5110 & 3.278 \\
\hline H59C & O58 $8^{10}$ & 2.721 & H59C & $\mathrm{N} 61^{10}$ & 3.538 \\
\hline H59C & $\mathrm{C} 65^{10}$ & 3.508 & H59C & $\mathrm{H} 50^{10}$ & 2.811 \\
\hline H59C & $\mathrm{H} 58^{10}$ & 2.662 & $\mathrm{H} 59 \mathrm{C}$ & $\mathrm{H} 65 \mathrm{~A}^{10}$ & 2.765 \\
\hline $\mathrm{H} 60 \mathrm{~A}$ & C $36^{1}$ & 3.391 & $\mathrm{H} 60 \mathrm{~A}$ & F66 ${ }^{3}$ & 3.382 \\
\hline $\mathrm{H} 60 \mathrm{~A}$ & $\mathrm{H} 12$ & 3.122 & $\mathrm{H} 60 \mathrm{~A}$ & H36 ${ }^{1}$ & 2.756 \\
\hline $\mathrm{H} 60 \mathrm{~A}$ & $\mathrm{H} 37^{1}$ & 3.181 & $\mathrm{H} 60 \mathrm{~A}$ & $\mathrm{H}^{2} 9 \mathrm{~B}^{10}$ & 3.574 \\
\hline H60B & $\mathrm{N} 48^{10}$ & 3.198 & H60B & C4910 & 3.569 \\
\hline $\mathrm{H} 60 \mathrm{~B}$ & N52 $2^{10}$ & 3.492 & $\mathrm{H} 60 \mathrm{~B}$ & $\mathrm{C} 53^{10}$ & 3.167 \\
\hline H60B & $\mathrm{Cl} 68^{9}$ & 3.224 & H60B & H36 ${ }^{1}$ & 3.056 \\
\hline H60B & $\mathrm{H} 37^{1}$ & 3.004 & $\mathrm{H} 60 \mathrm{C}$ & $\mathrm{N} 48^{10}$ & 3.180 \\
\hline $\mathrm{H} 60 \mathrm{C}$ & $\mathrm{C} 49^{10}$ & 3.020 & $\mathrm{H} 60 \mathrm{C}$ & $\mathrm{C} 50^{10}$ & 3.384 \\
\hline $\mathrm{H} 60 \mathrm{C}$ & N55 $5^{10}$ & 3.345 & $\mathrm{H} 60 \mathrm{C}$ & $058^{10}$ & 3.380 \\
\hline $\mathrm{H} 60 \mathrm{C}$ & C5910 & 3.382 & $\mathrm{H} 60 \mathrm{C}$ & $\mathrm{H} 55^{10}$ & 3.501 \\
\hline $\mathrm{H} 60 \mathrm{C}$ & $\mathrm{H} 58^{10}$ & 3.404 & $\mathrm{H} 60 \mathrm{C}$ & $H 59 B^{10}$ & 2.511 \\
\hline $\mathrm{H} 62 \mathrm{~A}$ & $C 4^{11}$ & 3.340 & $\mathrm{H} 62 \mathrm{~A}$ & $\mathrm{Cl} 67$ & 2.954 \\
\hline $\mathrm{H} 62 \mathrm{~A}$ & $\mathrm{H} 4^{11}$ & 2.978 & $\mathrm{H} 62 \mathrm{~A}$ & $\mathrm{H} 8 \mathrm{~B}$ & 2.905 \\
\hline $\mathrm{H} 62 \mathrm{~A}$ & $\mathrm{H} 21^{8}$ & 3.170 & H62B & $\mathrm{C} 21^{8}$ & 3.250 \\
\hline $\mathrm{H} 62 \mathrm{~B}$ & $\mathrm{Cl} 67$ & 3.459 & H62B & $\mathrm{Cl} \mid 68^{8}$ & 3.090 \\
\hline
\end{tabular}




\begin{tabular}{|c|c|c|c|c|c|}
\hline $\mathrm{H} 62 \mathrm{~B}$ & $\mathrm{H} 19^{8}$ & 3.525 & $\mathrm{H} 62 \mathrm{~B}$ & $\mathrm{H} 21^{8}$ & 2.420 \\
\hline H62B & $\mathrm{H} 56 \mathrm{~A}^{8}$ & 3.511 & H62B & $\mathrm{H} 59 \mathrm{~A}^{9}$ & 2.968 \\
\hline $\mathrm{H} 63$ & $\mathrm{~N} 6^{11}$ & 2.800 & $\mathrm{H} 63$ & $C 7^{11}$ & 3.496 \\
\hline H63 & $C 8^{11}$ & 3.547 & H63 & $\mathrm{N} 10^{8}$ & 2.991 \\
\hline $\mathrm{H} 63$ & $\mathrm{C} 11^{8}$ & 3.383 & $\mathrm{H} 63$ & $\mathrm{C} 12^{8}$ & 3.347 \\
\hline $\mathrm{H} 63$ & $\mathrm{H} 4^{11}$ & 3.373 & H63 & $\mathrm{H} 8 \mathrm{C}^{11}$ & 2.938 \\
\hline $\mathrm{H} 63$ & $\mathrm{H} 12^{8}$ & 3.020 & $\mathrm{H} 63$ & $\mathrm{H} 21^{8}$ & 3.071 \\
\hline $\mathrm{H} 64 \mathrm{~A}$ & C5 & 3.504 & $\mathrm{H} 64 \mathrm{~A}$ & N6 & 3.105 \\
\hline $\mathrm{H} 64 \mathrm{~A}$ & $N 6^{11}$ & 3.161 & $\mathrm{H} 64 \mathrm{~A}$ & $C 7^{11}$ & 3.305 \\
\hline $\mathrm{H} 64 \mathrm{~A}$ & $\mathrm{H} 8 \mathrm{~A}^{11}$ & 3.361 & $\mathrm{H} 64 \mathrm{~A}$ & $\mathrm{H} 8 \mathrm{C}^{11}$ & 3.564 \\
\hline H64B & F341 & 3.022 & H64B & $\mathrm{H} 8 \mathrm{~A}^{11}$ & 3.286 \\
\hline $\mathrm{H} 64 \mathrm{~B}$ & $\mathrm{H} 8 \mathrm{C}^{11}$ & 3.500 & H64B & $\mathrm{H} 12^{8}$ & 3.569 \\
\hline $\mathrm{H} 65 \mathrm{~A}$ & F341 & 2.518 & $\mathrm{H} 65 \mathrm{~A}$ & C59 ${ }^{9}$ & 3.135 \\
\hline $\mathrm{H} 65 \mathrm{~A}$ & $\mathrm{H} 59 \mathrm{~A}^{9}$ & 2.951 & $\mathrm{H} 65 \mathrm{~A}$ & $\mathrm{H} 59 \mathrm{~B}^{9}$ & 3.172 \\
\hline $\mathrm{H} 65 \mathrm{~A}$ & $\mathrm{H} 59 \mathrm{C}^{9}$ & 2.765 & H65B & $\mathrm{C} 4$ & 3.455 \\
\hline H65B & C5 & 2.772 & H65B & N6 & 2.989 \\
\hline H65B & $\mathrm{C} 7$ & 3.178 & $\mathrm{H} 65 \mathrm{~B}$ & $\mathrm{Cg}$ & 3.280 \\
\hline H65B & N10 & 3.170 & H65B & C11 & 2.870 \\
\hline H65B & C12 & 3.571 & H65B & F341 & 2.789 \\
\hline
\end{tabular}

Symmetry Operators:
(1) $X+1, Y, Z$
(2) $-X+2, Y+1 / 2,-Z$
(3) $X, Y+1, Z$
(4) $-X+1, Y+1 / 2,-Z$
(5) $X-1, Y, Z$
(6) $-X+1, Y+1 / 2-1,-Z$
(7) $-X+1, Y+1 / 2-1,-Z+1$
(8) $X, Y-1, Z$
(9) $-X+2, Y+1 / 2-1,-Z+1$
(10) $-X+2, Y+1 / 2,-Z+1$
(11) $-X+2, Y+1 / 2-1,-Z$
(12) $-X+1, Y+1 / 2,-Z+1$ 